Geological Society, London, Special Publications

\title{
A genetic link between silicic slab components and calc-alkaline arc volcanism in central Mexico
}

Susanne M. Straub, Georg F. Zellmer, Arturo Gómez-Tuena, Ramon Espinasa-Pereña, Ana Lillian Martin-del Pozzo, Finlay M. Stuart and Charles H. Langmuir

Geological Society, London, Special Publications 2014, v.385; p31-64.

doi: $10.1144 /$ SP385.14

$\begin{array}{ll}\begin{array}{l}\text { Email alerting } \\ \text { service }\end{array} & \begin{array}{l}\text { click here to receive free e-mail alerts when } \\ \text { new articles cite this article }\end{array} \\ \begin{array}{l}\text { Permission } \\ \text { request }\end{array} & \begin{array}{l}\text { click here to seek permission to re-use all or } \\ \text { part of this article }\end{array} \\ \text { Subscribe } & \begin{array}{l}\text { click here to subscribe to Geological Society, } \\ \text { London, Special Publications or the Lyell } \\ \text { Collection }\end{array}\end{array}$

Notes

(C) The Geological Society of London 2014

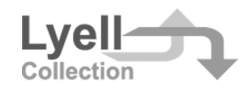




\title{
A genetic link between silicic slab components and calc-alkaline arc volcanism in central Mexico
}

\author{
SUSANNE M. STRAUB ${ }^{1 *}$, GEORG F. ZELLMER ${ }^{2,3}$, ARTURO GÓMEZ-TUENA $^{4}$, \\ RAMON ESPINASA-PEREÑA ${ }^{5}$, ANA LILLIAN MARTIN-DEL POZZO ${ }^{6}$, \\ FINLAY M. STUART ${ }^{7}$ \& CHARLES H. LANGMUIR ${ }^{8}$ \\ ${ }^{1}$ Lamont Doherty Earth Observatory at the Columbia University, \\ 61 Route 9W, Palisades NY 10964, USA \\ ${ }^{2}$ Institute of Earth Sciences, Academia Sinica, 128 Academia Road, Sec. 2, Nankang, \\ Taipei 11529, Taiwan, Republic of China \\ ${ }^{3}$ Present address: Institute of Agriculture and Environment, Massey University, \\ Palmerston North 4442, New Zealand
}

${ }^{4}$ Centro de Geociencias, Universidad Nacional Autónoma de México, Querétaro 76230, México

${ }^{5}$ Centro Nacional de Prevención de Desastres, Secretaría de Gobernación, Av. Delfín Madrigal 665, Col. Pedregal de Santo Domingo C.P. 04360, Delegación Coyoacán, México D.F., Mexico

${ }^{6}$ Instituto de Geofisica, Universidad Nacional Autónoma de México, Ciudad Universitaria, Mexico, D.F. 04510, Mexico

${ }^{7}$ Isotope Geosciences Unit, Scottish Universities Research and Reactor Centre, East Kilbride G75 OQF, UK

${ }^{8}$ Department of Earth and Planetary Sciences, Harvard University, 20 Oxford Street, Cambridge, MA 02137, USA

*Corresponding author (e-mail: smstraub@ldeo.columbia.edu)

\begin{abstract}
A fundamental question in the formation of orogenic andesites is whether their high melt $\mathrm{SiO}_{2}$ reflects the recycling of silicic melts from the subducted slab or the processing of basaltic mantle melts in the overlying crust. The latter model is widely favoured, because most arc magmas lack the 'garnet' signature of partial slab melts. Here we present new trace element data from Holocene high-Mg\# $>64-72$ calc-alkaline basalts to andesites $\left(50-62 \mathrm{wt} \% \mathrm{SiO}_{2}\right)$ from the central Mexican Volcanic Belt that crystallize high-Ni olivines with the high ${ }^{3} \mathrm{He} /{ }^{4} \mathrm{He}=7-8$ of the upper mantle. These magmas have been proposed to be partial melts from 'reaction pyroxenites', which formed by hybridization of mantle peridotite $(c .82-85 \%)$ and heavy rare earth elementdepleted silicic slab melt $(>15-18 \%)$. Forward and inverse models suggest that the absence of a garnet signature in these melts reflects the efficient buffering of the heavy rare earth elements (Ho to $\mathrm{Lu}$ ) in the subarc mantle. In contrast, all elements more incompatible than Ho - excepting $\mathrm{TiO}_{2}$ - are more or less strongly controlled by the silicic slab flux that also directly contributes to the silicic arc magma formation. Our study emphasizes the strong link between slab recycling and the genesis of orogenic andesites.
\end{abstract}

Supplementary material: Methods, additional data and modelling parameters are available at http://www.geolsoc.org.uk/SUP18686

Deciphering andesite petrogenesis at convergent margins is fundamental to understanding the differentiation of silicate Earth and the growth of the andesitic continental crust (Ringwood 1974; Gill 1981; Rudnick 1995). The plate tectonic model clearly links andesite genesis to plate subduction; however, it remains debated where and how andesitic arc magmas are produced. Low-degree partial melts from the subducted eclogitic slab are silicic (Beard 1995; Rapp \& Watson 1995; Skora \& Blundy 2010), but display strong garnet signatures (e.g. Pertermann \& Hirschmann 2003; Klimm et al. 2008). However, clear garnet signatures are comparatively rare in global arc magmas, 
and are mostly related to local or transient regional conditions (e.g. Kay 1978; Defant \& Drummond 1990; Yogodzinksi et al. 2001; Gomez-Tuena et al. 2007a). The missing garnet signature is therefore a key argument in models that consider formation of arc magmas through partial melting of a peridotite mantle that absorbed only trace amounts (c. 1-2 wt \%) of a highly enriched slab component. While this slab component may control the budget of the fluid-mobile, highly incompatible elements in arc magmas (Morris et al. 1990; Plank \& Langmuir 1993; Elliott et al. 1997), it imparts no garnet signature. Moreover, the model implies that initial arc melts are typical partial peridotite melts with low $\mathrm{SiO}_{2}(<50 \mathrm{wt} \%)$ and high $\mathrm{MgO}(>10 \mathrm{wt} \%)$ abundances (e.g. Langmuir et al. 1992). Because the abundances of melt water in arc magmas are generally too low to cause more than a moderate increase of melt $\mathrm{SiO}_{2}$ in the initial mantle melts (e.g. Baker et al. 1994; Ruscitto et al. 2012), the evolution to andesitic, dacitic and rhyolitic magmas must then take place in the overlying crust, by fractional crystallization or crustal assimilation, or a mixture of both (e.g. Eichelberger 1978; Hildreth \& Moorbath 1988; Plank \& Langmuir 1988; Tamura \& Tatsumi 2002; Reubi \& Blundy 2008, 2009).

While this view of andesite genesis is widely accepted, comprehensive datasets that combine detailed geological information with high-quality geochemical data reveal major problems. An excellent example is provided by the monogenetic volcanoes of the Holocene Sierra Chichinautzin Volcanic Field in the central Mexican Volcanic Belt (MVB) which have been the object of many detailed studies in recent years (e.g. Wallace \& Carmichael 1999; Straub \& Martin-Del Pozzo 2001; Siebe et al. 2004a; Schaaf et al. 2005; Straub et al. 2008, 2011; Agustín-Flores et al. 2011). Many of the Sierra Chichinautzin volcanoes erupt olivinerich, high-Mg\# (up to 74) and high $\mathrm{MgO}$ (up to $10 \mathrm{wt} \%$ ) calc-alkaline basalts to andesites that display the arc-typical enrichments of fluid-mobile large-ion lithophile elements (LILE) relative to the high-field-strength elements (HFSE) and rare earth elements (REE). Intriguingly, the overall $\mathrm{SiO}_{2}$ increase from basalt to andesite cannot be attributed to a fractional crystallization link, as basalt to andesite series have different ${ }^{87} \mathrm{Sr} /{ }^{86} \mathrm{Sr}$ and ${ }^{143} \mathrm{Nd} /$ ${ }^{144} \mathrm{Nd}$ isotope ratios (e.g. Fig. 1a, b; Wallace \& Carmichael 1999; Schaaf et al. 2005). On the other hand, the linear, continuous trends of major and trace elements with melt $\mathrm{SiO}_{2}$ suggests mixing of basaltic and silicic compositions (Fig. 1a). The magmas erupt through $c$. $45 \mathrm{~km}$-thick continental crust (e.g. Kim et al. 2010), and thus the silicic end member may be a silicic component from the ambient crust. If this was the case, huge amounts of silicic crust on the order of tens of per cent must be incorporated in the intermediate andesites (Fig. 1a). Such amounts, however, are improbable, because the Mexican high-Mg\# andesites are both unusually primitive in radiogenic isotopes and rich in $\mathrm{Ni}$ (up to $250 \mathrm{ppm}$ ) and $\mathrm{Cr}$ (up to $500 \mathrm{ppm}$ ). Moreover, the high-Mg\# magmas commonly crystallize fosteritic olivines with the high ${ }^{3} \mathrm{He} /{ }^{4} \mathrm{He}=$ 7-8 $\mathrm{R}_{\mathrm{a}}$ typical of mantle melts (Fig. 1, Straub et al. 2011, 2013). In view of the extreme sensitivity of low-He mantle melts towards the assimilation of ${ }^{4} \mathrm{He}$-rich crustal material with ${ }^{3} \mathrm{He} /{ }^{4} \mathrm{He}<1$, these melts can have assimilated only trace amounts of crustal material $(<0.0001 \%$, curve b in Fig. 1c; Straub et al. 2011, 2013).

The high ${ }^{3} \mathrm{He} /{ }^{4} \mathrm{He}$ olivines also have unusual high-Ni abundances which provide important clues to magma genesis. The $\mathrm{Ni}$ of the Mexican olivine exceeds by far the Ni of olivines from partial peridotite melts (Fig. 1d). Straub et al. (2011) proposed that such high-Ni olivines may reflect the presence of silica-deficient and silica-excess 'reaction pyroxenites' in the mantle wedge that formed through reaction of silicic slab components with the ambient peridotite mantle (Straub et al. 2008, 2011). Upon melting, such pyroxenite segregations may produce a broader range of high- $\mathrm{Mg \#}$ basaltic and dacitic initial component melts. Mixing of many of these melts during ascent through mantle and crust then produces a series of high-Mg\# basaltic to andesitic and dacitic melt that only experienced minor modification (olivine loss and recharge melt mixing) in the overlying crust (Straub et al. 2011). In other words, this model suggests that Mexican arc andesites are principally hybrids of slab and mantle components, and do not form through crustal differentiation.

While this 'pyroxenite model' accounts well for the major element characteristics (Straub et al. 2008, 2011, 2013), it is not clear how it influences the trace elements. Any 'reaction pyroxenite' that gives rise to silicic mantle melts must contain at least $c$. $15-18 \%$ of a silicic slab component. This is the minimum amount required to transform peridotite to 'reaction pyroxenite'. The obvious question is how this large amount (and more) of slab material reconciles with incompatible trace element of the central MVB calc-alkaline magmas that with rare exceptions - have no garnet signatures (LaGatta 2003; Schaaf et al. 2005; Straub et al. 2008). The purpose of this paper is to discuss the systematics of incompatible trace elements in the context of the 'pyroxenite model'. While we focus on basaltic to andesite calc-alkaline high-Mg\# $(>64)$ magmas that have high-Ni and high ${ }^{3} \mathrm{He} /$ ${ }^{4} \mathrm{He}$ olivines and that are least affected by crustal differentiation, these data are discussed in the context of all central MVB calc-alkaline magmas. 


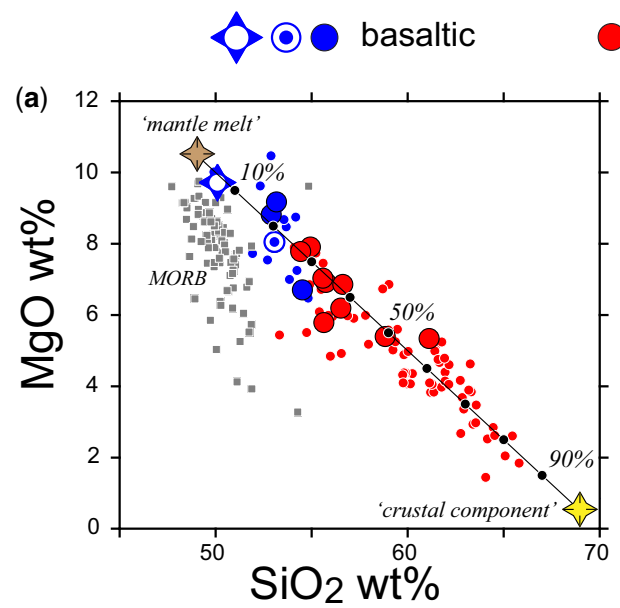
andesitic
MORB
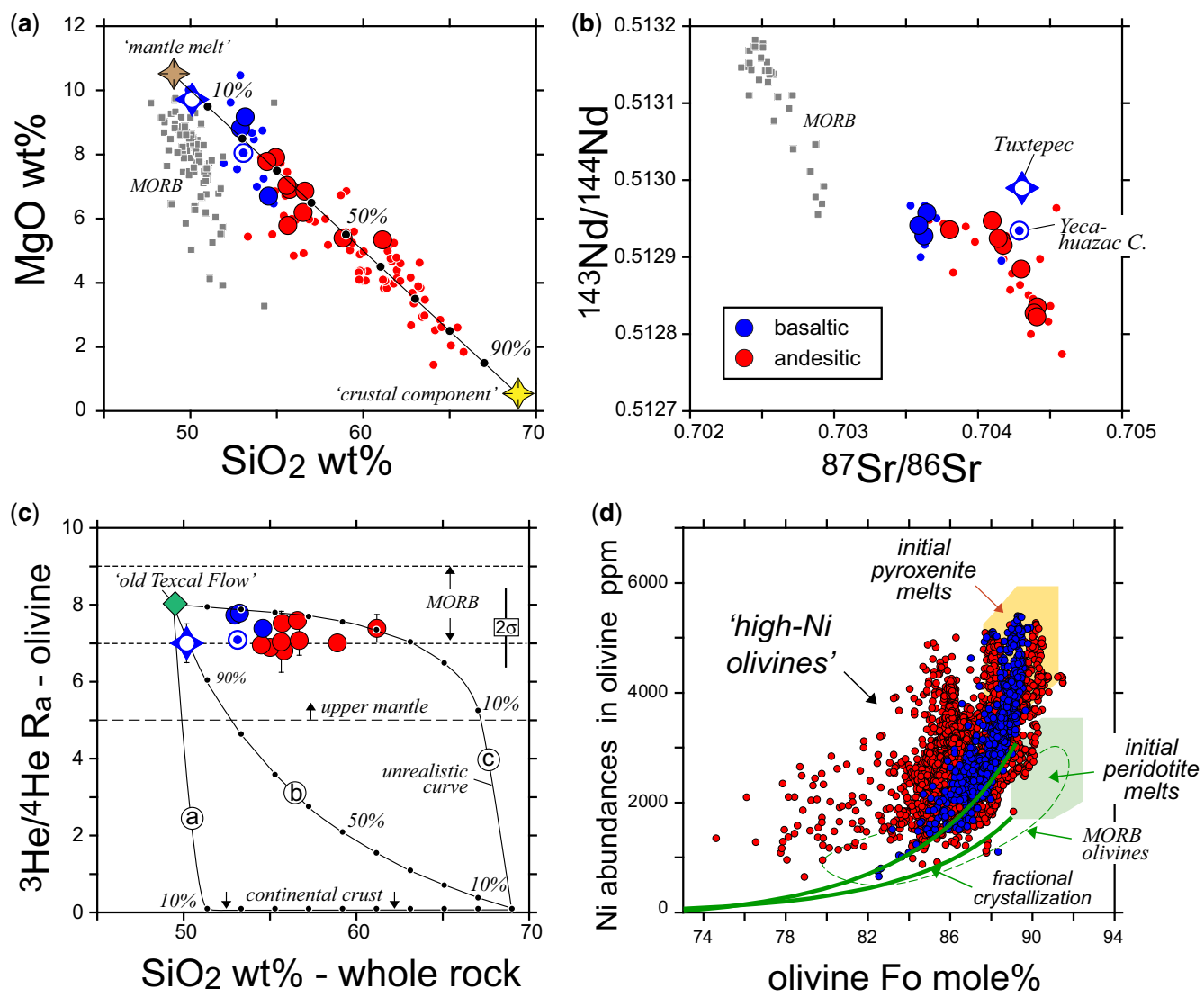

Fig. 1. Summary of previous studies on calc-alkaline magmas in the central Mexican Volcanic Belt (MVB). MORB are from PetDB (2011); for other data sources see text. Basaltic and andesitic magmas are quartz-normative and olivine-normative magmas respectively (as discussed in text). Large symbols in $(\mathrm{a}-\mathrm{c})$ denote samples for which ${ }^{3} \mathrm{He} /{ }^{4} \mathrm{He}$ has been obtained. (a) Whole-rock $\mathrm{SiO}_{2} \mathrm{v} . \mathrm{MgO}$ (in wt $\%$ ). Linear mixing curve assumes mixing between a basaltic melt $\left(50 \mathrm{wt} \% \mathrm{SiO}_{2}, 10 \mathrm{wt} \% \mathrm{MgO}\right)$ and a silicic crustal component (69 wt $\left.\% \mathrm{SiO}_{2}, 0.5 \mathrm{wt} \% \mathrm{MgO}\right)$. (b) Whole-rock ${ }^{87} \mathrm{Sr} /{ }^{86} \mathrm{Sr}$ v. ${ }^{143} \mathrm{Nd} /{ }^{144} \mathrm{Nd}$. (c) Whole-rock $\mathrm{SiO}_{2}(\mathrm{wt} \%)$ v. olivine ${ }^{3} \mathrm{He} /{ }^{4} \mathrm{He}\left(\mathrm{R}_{\mathrm{a}}\right)$. External precision of ${ }^{3} \mathrm{He} /{ }^{4} \mathrm{He}$ is $\pm 1 \mathrm{R}_{\mathrm{a}}$. 'Old Texcal Flow' from Straub et al. (2011) has the highest ${ }^{3} \mathrm{He} /{ }^{4} \mathrm{He}=8 \mathrm{R}_{\mathrm{a}}$ in central MVB and is taken as proxy to ${ }^{3} \mathrm{He} /{ }^{4} \mathrm{He}$ of mantle melt. Because crustal $\mathrm{He}$ is by four orders of magnitude higher than He in mantle melts, the mixing curve (curve a) clearly misses the data if only traces of crustal material were assimilated. Curve $b$ assumes loss of $99.99 \%$ of the crustal He. Stippled curve c assumed that crustal material has only c. $6 \%$ of mantle $\mathrm{He}$ which is not realistic (Ballentine \& Burnard 2002). See Supplementary material for more details on $\mathrm{SiO}_{2}-{ }^{3} \mathrm{He} /{ }^{4} \mathrm{He}$ mixing curves. (d) Olivine $\mathrm{Ni}(\mathrm{ppm}) \mathrm{v}$. Fo (mole\%) of olivines with high ${ }^{3} \mathrm{He} /{ }^{4} \mathrm{He}=7-8 \mathrm{R}_{\mathrm{a}}$. Green field indicates olivines in equilibrium with initial mantle melts from peridotite, thick green lines are the liquid lines of descent for olivines crystallizing from peridotite melts. Ochre field indicates olivines in equilibrium with initial mantle melts from 'reaction pyroxenites'. Modified from Straub et al. (2011). Data from Straub et al. (2011, this study).

\section{Geological background}

\section{Central Mexican Volcanic Belt}

The geological setting of the Mexican Volcanic Belt has been described in detail elsewhere (e.g. Gómez-Tuena et al. 2007b; Ferrari et al. 2011), and only a short summary is given here. The MVB is a Pliocene-Quaternary volcanic arc that is related to the subduction of the Cocos and Rivera plates along the Middle American Trench (Fig. 2). The Middle American trench runs oblique at an angle of $c .17^{\circ}$ to the MVB, owing to the nearhorizontal subduction of the slab beneath the 
forearc in the central-eastern arc (Pardo \& Suarez 1995). In the central MVB, the arc front is located at c. $300-350 \mathrm{~km}$ from trench, where the slab steepens abruptly to $65-75^{\circ}$ (Perez-Campos et al. 2008). The central segment of the MVB between composite volcanoes Popocatepetl and Nevado de Toluca $\left(c .100-98^{\circ} 30^{\prime} \mathrm{W}\right)$ is constructed on $c$. $45 \mathrm{~km}$ thick sialic crust of Proterozoic granulites overlain by Jurassic metapelites, granites and Mesozoic limestones (Kim et al. 2010; Ortega-Gutiérrez et al. 2012).

Subduction zone parameters, such as slab age, slab dip and crustal thickness and composition, are constant in central MVB. All the same, Holocene volcanoes Nevado de Toluca, Popocatepetl and the volcanoes of the interjacent Sierra Chichinautzin Monogenetic Field erupt calc-alkaline basaltic to dacitic magmas with highly variable trace element patterns that include a subordinate group of mildly alkaline Nb-rich basalts and basaltic andesites (e.g. Verma 1999; Wallace \& Carmichael 1999; LaGatta 2003; Siebe et al. 2004a; Schaaf et al. 2005). The latter are referred to as 'OIB-type' or high-'Nb arc basalts' (HNAB), as their trace element patterns resemble those of intraplate magmas. The far more abundant calc-alkaline series are characterized by strong enrichments of LILE relative to REE and HFSE and strongly resemble average continental crust. Together with the HNABs, the calc-alkaline magmas form a continuum in compositional space with many transitional compositions, but no 'natural' boundary (Verma 1999; Wallace \& Carmichael 1999; LaGatta 2003; Schaaf et al. 2005). Following LaGatta (2003), all magmas with $<\mathrm{Nb}=16 \mathrm{ppm}$ are considered as calc-alkaline magmas. The HNAB have $>16-$ $35 \mathrm{ppm} \mathrm{Nb}$, and are discussed in detail elsewhere (Straub et al. 2013).

\section{Sampling strategy}

Samples for this study are from the Sierra Chichinautzin Volcanic Field, in addition to a few samples from Popocatepetl volcano. Sample collection took place during multiple field trips in the years 2004-2009. The field trips were spaced about a year apart, which allowed for intermittent major and trace element analyses that then guided further field work. Originally, we searched for zoned monogenetic volcanoes as prime targets for studying the causes of melt differentiation. While this approach was successful for the HNAB (Straub et al. 2013), the calc-alkaline monogenetic volcanoes were either nearly homogeneous (e.g. Pelado, Tlaloc, Siebe et al. 2004a, 2005; Schaaf et al. 2005), or the eruptive sequence could not be, or could only partially be, reconstructed (e.g. Cuatepel, Guespalapa, this study). Several monogenetic volcanoes erupt both calc-alkaline and HNAB magmas, but without significant zoning in either group (e.g. Yecahuazac, Suchiooc, this study).

Calc-alkaline magmas comprise basalts, basaltic andesites, andesites and dacites. Many calcalkaline basalts to andesites have high values of $\mathrm{Mg \#}>64$, fairly high $\mathrm{Ni}>100 \mathrm{ppm}$ and $\mathrm{Cr}>155$ ppm, and olivine as single, or dominant, phenocryst regardless of the broad $\mathrm{SiO}_{2}$ range. For simplicity, the calc-alkaline series are divided into two groups based on their normative composition: a 'basaltic' (olivine-normative) and 'andesitic' (quartz-normative) group (Table 1, Straub et al. 2011). Olivine phenocrysts in both basaltic and andesitic magmas have been analysed for ${ }^{3} \mathrm{He} /{ }^{4} \mathrm{He}$ (Straub et al. 2011). These 'high $-{ }^{3} \mathrm{He} /{ }^{4} \mathrm{He}$ samples' are denoted by large, filled blue and red circles in the figures, and originate from monogenetic volcanoes Cuatepel (two samples), Guespalapa (seven), Suchiooc Cone (two), Tuxtepec (one) and Yecahuazac (one). One sample is from Popocatepetl. All other calc-alkaline basalts and andesites are shown as small blue and red circles.

The samples displayed are either from our own field work, or have been published by Straub et al. (2008, 2011, 2013) and LaGatta (2003). Sample locations are shown in Figure 2 and geographical coordinates are given in Table 1. This sample set fully represents the spectrum of the central MVB magmas and is free from interlaboratory bias. For these reasons and for clarity, other published compositional data on central MVB volcanics were omitted from the plots (e.g. Wallace \& Carmichael 1999; Siebe et al. 2004a, 2005; Schaaf et al. 2005; Meriggi et al. 2008; Agustín-Flores et al. 2011).

All new data are listed in Table 1. Many samples were taken from the same volcanoes that have been selected for the ${ }^{3} \mathrm{He} /{ }^{4} \mathrm{He}$ work, which includes Popocatepetl (six additional samples), Cuatepel (two), Guespalapa (12), Suchiooc (10), Yecahuazac (three) and Tuxtepec (one). In addition, we report data for samples from Sierra Chichinautzin monogenetic volcanoes Cuatzin (one sample), Ocusacayo (two), Pelado (three), Teuthli (two), Tlaloc (two), Muñeco (one) and a lava flow in proximity to (but not from) Tuxtepec Cone (one). Subvolcanic rocks were analysed from Zempoala Ridge (two) and from Cerro Magdalena (one).

\section{Sample locations}

In the following, the volcanoes with the high ${ }^{3} \mathrm{He} /{ }^{4} \mathrm{He}$ samples are briefly described. Volcan Cuatepel is located just east of the village of Juchitepec and halfway between the central Sierra Chichinautzin and Popocatepetl volcano. Cuatepetl magmas are quartz-normative, and produced a scoria cone of olivine-phyric, dark-grey andesite bombs 


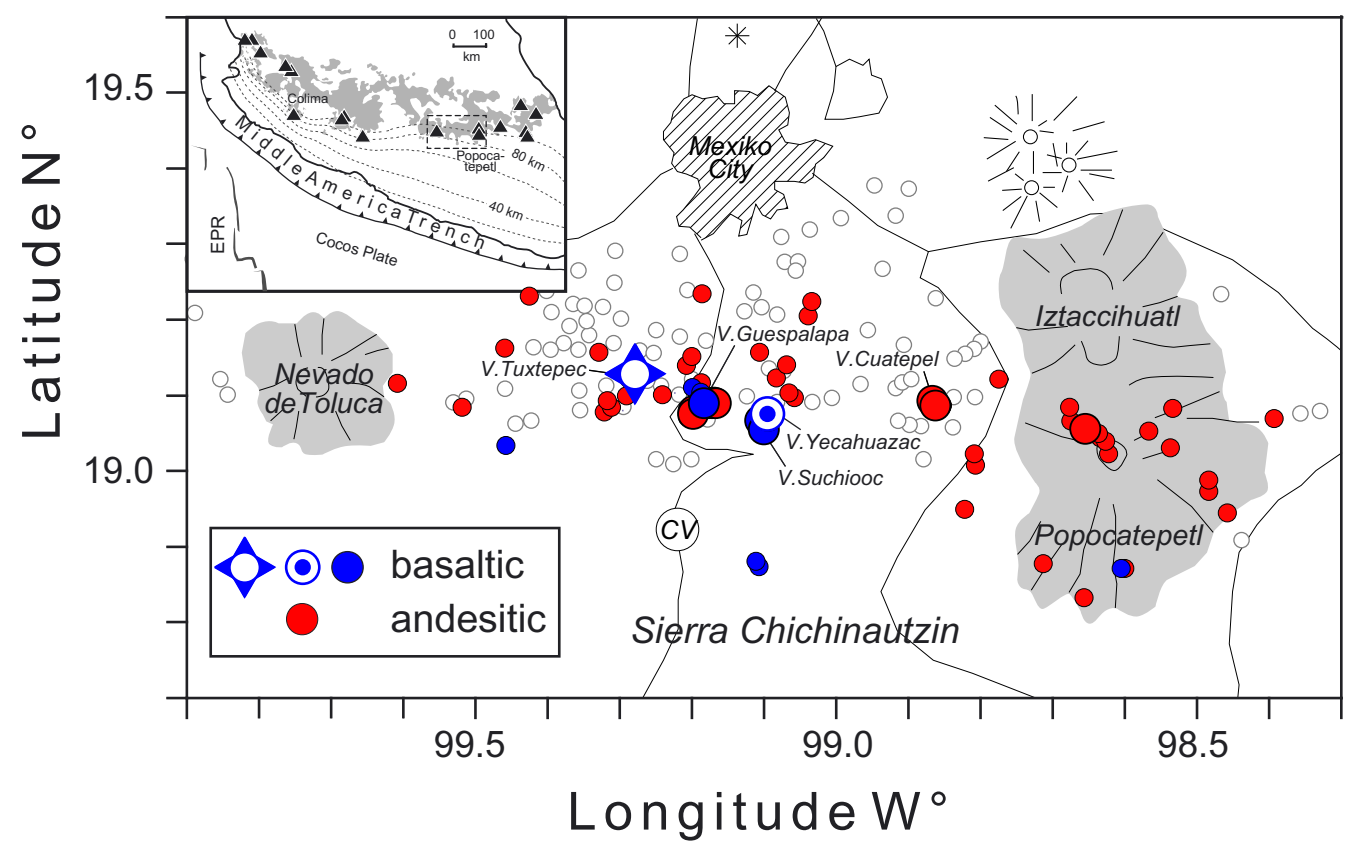

Fig. 2. Geological setting of the central Mexican Volcanic Belt. Inset shows position of study area within the Mexican subduction zone. Slab contours after Pardo \& Suarez (1995). The enlargement shows the monogenetic volcanoes (small open grey circles) of the Sierra Chichinautzin that is flanked by Quaternary composite volcanoes Nevado de Toluca and Popocatepetl. Only calc-alkaline compositions $(\mathrm{Nb}<16 \mathrm{ppm})$ are shown. Large symbols are samples with high- ${ }^{3} \mathrm{He} /{ }^{4} \mathrm{He}=7-8 \mathrm{R}_{\mathrm{a}}$ olivine; small symbols are other samples (this study and published data). See text for further details.

$\left(58 \mathrm{wt} \% \mathrm{SiO}_{2}\right)$, and an olivine-rich basaltic andesite flow $\left(56 \mathrm{wt} \% \mathrm{SiO}_{2}\right)$ at the base of the western flank. The youthful appearance suggests a Holocene age. There is no information about the relative succession of cone and flow.

The Guespalapa Volcanic Complex formed between 2.8 and $4.7 \mathrm{ka} \mathrm{BP}$ (Siebe et al. 2004a, $b$ ). All magmas are olivine-phyric. It is a broad shield volcano that is topped by three scoria cones: El Caballito, with multiple vents, and cones Manteca and El Hoyo. The Guespalapa shield consists of quartz-normative basaltic andesites to andesites with $c$. 55.4-61.2 wt\% $\mathrm{SiO}_{2}$ and low $\mathrm{Nb}(=4.1-$ $8.8 \mathrm{ppm})$. The three scoria cones erupt quartznormative basaltic andesites, with the exception of El Caballito, which produces both quartz-normative (55.4 wt\% $\mathrm{SiO}_{2}$ ) and olivine-normative basaltic andesites (54.4-54.8 wt\% $\mathrm{SiO}_{2}$ ). El Caballito has also relatively high $\mathrm{Nb}=10.8-13.6 \mathrm{ppm}$ that is more typical for transitional rather than calc-alkaline compositions. Manteca $\left(56.7 \mathrm{wt} \% \mathrm{SiO}_{2}\right.$ ) and El Hoyo (54.9 wt\% $\mathrm{SiO}_{2}$ ) are the most silicic Guespalapa magmas. El Hoyo $(\mathrm{Mg} \#=69.8)$ and Manteca $(\mathrm{Mg \#}=66.2)$ have higher values of $\mathrm{Mg \#}$ than El Caballito $(\mathrm{Mg} \#=65.0)$, but much lower $\mathrm{Nb}=3.5-4.2 \mathrm{ppm}$. There is no information about the temporal succession except that Manteca and El Caballito formed after the Guespalapa lava shield.

Volcan Suchiooc is a scoria cone that is associated with lavas flows of up to $10 \mathrm{~km}$ length (Espinasa-Perena 2006). All magmas are olivinephyric. The lava flows were emplaced during the Brunhes Chron and hence are younger than $<0.73-0.79 \mathrm{Ma}$ (Urrutia-Fucugauchi \& Martin del Pozzo 1993). Calc-alkaline basaltic andesites $\left(53.0-54.2 \mathrm{wt} \% \mathrm{SiO}_{2}, \mathrm{Nb}=10.2-12.9 \mathrm{ppm}\right)$ can be found at Suchiooc Cone and proximal lava flows within a $c .1 \mathrm{~km}$ perimeter are olivine-normative. Olivine-normative basaltic HNABs $(>16 \mathrm{ppm}$ $\mathrm{Nb})$ seem to have been emplaced after the calcalkaline basalts. They extrude within a few hundred metres from Suchiooc Cone and make up all distal lava flows.

Like Suchiocc, Volcan Yecahuazac consists of an olivine-normative calc-alkaline cone (53.1 wt $\% \mathrm{SiO}_{2} ; \mathrm{Nb}=8.4 \mathrm{ppm}$ ) and olivine-normative HNABs basaltic lava flows that breach through the southwestern flank of the cones and seem to postdate cone formation. All magmas are olivinephyric. Our field observations and data show that a lava flow emplaced in front of the 


\begin{tabular}{|c|c|c|c|c|c|c|c|c|c|c|c|c|c|c|c|c|c|c|c|c|c|c|c|}
\hline Volcano & Stratigraphic Unit & Sample_ID & Longitude $\mathrm{W}$ & Latitude $\mathrm{N}$ & Laboratory $^{\mathrm{a}}$ & Group $^{\mathrm{b}}$ & iroup ${ }^{c}$ & $\begin{array}{l}{ }^{3} \mathrm{He}^{4} / \\
{ }^{4} \mathrm{He}^{\mathrm{d}}\end{array}$ & Data source ${ }^{e}$ & $\mathrm{SiO}_{2}$ & $\mathrm{TiO}_{2}$ & $\mathrm{Al}_{2} \mathrm{O}_{3}$ & $\mathrm{Fe}_{2} \mathrm{O}_{3}^{\mathrm{t}}$ & $\mathrm{MnO}$ & $\mathrm{MgO}$ & $\mathrm{CaO}$ & $\mathrm{Na}_{2} \mathrm{O}$ & $\mathrm{K}_{2} \mathrm{O}$ & $\mathrm{P}_{2} \mathrm{O}_{5}$ & LOI & Sum & $\operatorname{Mg} \#^{f}$ & $\mathrm{Qtz}^{\mathrm{g}}$ \\
\hline opocatepetl & $\begin{array}{l}\text { Jentorillo aphyric } \\
\text { dacite }\end{array}$ & CH-09-18 & $98^{\circ} 38^{\prime} 04^{\prime \prime}$ & $19^{\circ} 02^{\prime} 39^{\prime \prime}$ & WSU/CGEO & Andesitic & Calc-alk. & & This study & 62.85 & 0.78 & 15.78 & 5.39 & 0.09 & 3.69 & 4.90 & 4.23 & 2.08 & 0.23 & & 100.02 & 60.9 & 13.6 \\
\hline pocatepetl & $\begin{array}{l}\text { ykke in upper } \\
\text { B.Nexpayantla }\end{array}$ & H-09-19 & $98^{\circ} 38^{\prime} 04^{\prime \prime}$ & $19^{\circ} 02^{\prime} 39^{\prime \prime}$ & WSU/CGEO & Andesitic & Calc-alk. & & This study & 64.07 & 0.47 & 15.98 & 3.87 & 0.06 & 1.44 & 3.89 & 4.24 & 1.99 & 0.15 & & 96.16 & 45.9 & 20.8 \\
\hline Popocatepetl & $\begin{array}{l}\text { Volcaniclast in upper } \\
\text { B.Nexpayantla }\end{array}$ & CH-09-20 & $98^{\circ} 38^{\prime} 04^{\prime \prime}$ & $19^{\circ} 02^{\prime} 39^{\prime \prime}$ & WSU/CGEO & Andesitic & Calc-alk. & & This study & 63.41 & 0.74 & 16.42 & 4.84 & 0.08 & 2.93 & 4.79 & 4.38 & 1.75 & 0.19 & & 99.52 & 58.0 & 15.7 \\
\hline Popocatepetl & $\begin{array}{l}\text { Volcaniclast } \\
\text { B.Nexpay }\end{array}$ & CH-09-21 & $98^{\circ} 38^{\prime} 04^{\prime \prime}$ & $19^{\circ} 02^{\prime} 39^{\prime \prime}$ & WSU/CGEO & Andesitic & Calc-alk. & & This study & 59.76 & 0.82 & 15.85 & 5.51 & 0.09 & 4.10 & 5.30 & 4.02 & 1.70 & 0.20 & & 97.35 & 62.8 & 11.9 \\
\hline Popocatepetl & $\begin{array}{l}\text { Dyke in lower } \\
\text { B. Nexpayant }\end{array}$ & CH-09-22 & $98^{\circ} 38^{\prime} 04^{\prime \prime}$ & $19^{\circ} 02^{\prime} 39^{\prime \prime}$ & WSU/CGEO & Andesitic & Calc-alk. & & This study & 56.54 & 0.89 & 16.72 & 6.30 & 0.10 & 4.92 & 7.07 & 4.07 & 1.22 & 0.21 & & 97.41 & 64.0 & 5.7 \\
\hline Popocatepetl & Nealtican flows & Popo5 & $98^{\circ} 32^{\prime} 14^{\prime \prime}$ & $19^{\circ} 01^{\prime} 51^{\prime \prime}$ & Harvard & Andesitic & Calc-alk. & & This study & 61.36 & 0.90 & 16.29 & 5.68 & 0.09 & 3.84 & 5.23 & 4.33 & 1.75 & 0.24 & & 99.72 & 60.6 & 11.8 \\
\hline & & & & & & $\begin{array}{l}\text { Andesitic } \\
\text { Andesitic }\end{array}$ & $\begin{array}{l}\text { Calc-alk. } \\
\text { Calc-alk. }\end{array}$ & & & $\begin{array}{l}55.59 \\
58.77\end{array}$ & $\begin{array}{l}0.94 \\
0.91\end{array}$ & $\begin{array}{l}16.26 \\
16.21\end{array}$ & $\begin{array}{l}7.21 \\
6.24\end{array}$ & $\begin{array}{l}0.12 \\
0.11\end{array}$ & $\begin{array}{l}7.46 \\
5.40\end{array}$ & $\begin{array}{l}7.01 \\
5.89\end{array}$ & $\begin{array}{l}3.55 \\
3.97\end{array}$ & $\begin{array}{l}1.25 \\
1.49\end{array}$ & $\begin{array}{l}0.19 \\
0.21\end{array}$ & & $\begin{array}{l}98.84 \\
98.57\end{array}$ & $\begin{array}{l}70.2 \\
66.3\end{array}$ & $\begin{array}{l}3.1 \\
8.4\end{array}$ \\
\hline Guespalapa & $\begin{array}{l}\text { Guespalapa shield } \\
\text { lava }\end{array}$ & CH-09-1 & $99^{\circ} 11^{\prime} 55^{\prime \prime}$ & $19^{\circ} 04^{\prime} 45^{\prime \prime}$ & WSU/CGEO & Andesitic & Calc-alk. & & This study & 55.43 & 1.20 & 17.09 & 7.42 & 0.11 & 5.86 & 6.79 & 4.31 & 1.10 & 0.26 & & 99.56 & 64.2 & 2.0 \\
\hline Guespalapa & $\begin{array}{l}\text { Guesp } \\
\text { lav }\end{array}$ & CH-07-8 & $99^{\circ} 10^{\prime} 26^{\prime \prime}$ & $19^{\circ} 05^{\prime} 26^{\prime \prime}$ & WSU/CGEO & Andesitic & Calc-alk. & & This study & 55.49 & 0.98 & 17.19 & 7.32 & 0.12 & 6.72 & 7.53 & 3.90 & 0.98 & 0.18 & & 99.69 & 67.6 & 2.1 \\
\hline Guespalapa & $\begin{array}{l}\text { Guespalapa shield } \\
\text { lava }\end{array}$ & CH-09-2 & $99^{\circ} 11^{\prime} 52^{\prime \prime}$ & $19^{\circ} 04^{\prime} 49^{\prime \prime}$ & WSU/CGEO & Andesitic & Calc-alk. & * & $\begin{array}{l}\text { Straub et al. } \\
\quad \text { (2011); this } \\
\text { study }\end{array}$ & 55.71 & 1.20 & 17.19 & 7.28 & 0.11 & 5.79 & 6.80 & 4.33 & 1.12 & 0.25 & & 99.78 & 64.4 & 2.3 \\
\hline uespalapa & $\begin{array}{l}\text { Guespalapa shield } \\
\text { lava }\end{array}$ & S12A & & $19^{\circ} 04^{\prime} 18^{\prime \prime}$ & Harvard & Andesitic & alc-alk. & & This study & 55.80 & 1.18 & 17.12 & 7.09 & 0.11 & 5.88 & 6.66 & 4.26 & 1.13 & 0.28 & & 99.52 & 65.4 & 2.9 \\
\hline Guespalapa & $\begin{array}{l}\text { Guespalapa shield } \\
\text { lava }\end{array}$ & CH-07-9 & $99^{\circ} 10^{\prime} 20^{\prime \prime}$ & $19^{\circ} 05^{\prime} 26^{\prime \prime}$ & WSU/CGEO & Andesitic & Calc-alk. & * & $\begin{array}{l}\text { Straub et al. } \\
\quad \text { (2011); this } \\
\text { study }\end{array}$ & 55.81 & 0.98 & 17.24 & 7.34 & 0.12 & 6.92 & 7.58 & 3.87 & 1.01 & 0.17 & & 100.31 & 68.2 & 2.1 \\
\hline Guespalapa & $\begin{array}{l}\text { Guespalapa shield } \\
\text { lava }\end{array}$ & H-05-11 & $99^{\circ} 11^{\prime} 18^{\prime \prime}$ & $19^{\circ} 05^{\prime} 22^{\prime \prime}$ & WSU/CGEO & Andesitic & Calc-alk. & & This study & 55.86 & 1.27 & 17.26 & 7.31 & 0.12 & 6.00 & 6.78 & 4.41 & 1.12 & 0.27 & 0.17 & 100.57 & 65.1 & 1.8 \\
\hline Guespalapa & lapa shield & H-05-14 & $99^{\circ} 12^{\prime} 07^{\prime \prime}$ & $19^{\circ} 04^{\prime} 33^{\prime \prime}$ & WSU/CGEO & Andesitic & Calc-alk. & & This study & 56.09 & 1.19 & 17.26 & 7.16 & 0.11 & 5.96 & 6.83 & 4.33 & 1.13 & 0.25 & 0.42 & 100.73 & 65.4 & 2.3 \\
\hline Guespalapa & $\begin{array}{l}\text { Guespal: } \\
\text { lava }\end{array}$ & CH-08-3 & $99^{\circ} 11^{\prime} 56^{\prime \prime}$ & $19^{\circ} 04^{\prime} 34^{\prime \prime}$ & WSU/CGEO & Andesitic & Calc-alk. & * & $\begin{array}{l}\text { Straub et al. } \\
\quad \text { (2011); this } \\
\text { study }\end{array}$ & 61.18 & 0.80 & 15.71 & 5.84 & 0.10 & 5.35 & 5.45 & 4.20 & 1.60 & 0.22 & & 100.45 & 67.6 & 10.3 \\
\hline & & & & & & & & & & & & & & & & & & & & & & & \\
\hline & & & & & WSU/CGEO & Basaltic & & * & Stra & 54.58 & 1.48 & 17.04 & 8.10 & 0.13 & 6.71 & 7.28 & 4.26 & 1.14 & 0.32 & 0.25 & 100.22 & 65.3 & \\
\hline & & & & & & & & & & & & & & & & & & & & 0.08 & & & \\
\hline & & & & & & & & & & & & & & & & & & & & & & & 1.0 \\
\hline Guespalapa & Manteca bomb & CH-07-6 & $99^{\circ} 10^{\prime} 38^{\prime \prime}$ & $19^{\circ} 05^{\prime} 26^{\prime \prime}$ & WSU/CGEO & Andesitic & Calc-alk. & * & $\begin{array}{r}\text { Straub } \\
(20 \\
\text { stuc }\end{array}$ & 56.58 & 1.01 & 17.48 & 7.15 & 0.11 & 6.20 & 7.12 & 4.09 & 1.02 & 0.18 & & 100.24 & 66.4 & 3.3 \\
\hline & & & & & & & & & & & & & & & & & & & & & & & 5 \\
\hline & o crater rim & & & & & & & & & & & & & & & & & & & & & 69.7 & 0.6 \\
\hline Guespalapa & $\begin{array}{l}\text { El Hoyo crater rim } \\
\text { lava }\end{array}$ & СH-07-12 & $99^{\circ} 10^{\prime} 10^{\prime \prime}$ & $19^{\circ} 05^{\prime} 29^{\prime \prime}$ & WSU/CGEO & Andesitic & Calc-alk. & * & $\begin{array}{l}\text { Straub et al. } \\
\text { (2011); this } \\
\text { study }\end{array}$ & 54.99 & 0.95 & 16.76 & 7.77 & 0.13 & 7.92 & 7.86 & 3.73 & 0.88 & 0.16 & & 100.37 & 69.9 & 0.6 \\
\hline & $\begin{array}{l}\text { El Hoyo crater rim } \\
\text { lava }\end{array}$ & & & & & & & & & 55.00 & 0.95 & 16.82 & 7.77 & 0.13 & 7.93 & 7.91 & 3.71 & 0.90 & 0.15 & & 100.50 & 69.9 & 0.5 \\
\hline Guespalapa & $\begin{array}{l}\text { El Hoyo crater rim } \\
\text { lava }\end{array}$ & СH-07-10 & $99^{\circ} 10^{\prime} 11^{\prime \prime}$ & $19^{\circ} 05^{\prime} 26^{\prime \prime}$ & WSU/CGEO & Andesitic & Calc-alk. & & This study & 55.22 & 0.95 & 16.90 & 7.78 & 0.13 & 7.74 & 7.88 & 3.78 & 0.89 & 0.16 & & 100.66 & 69.4 & 0.7 \\
\hline
\end{tabular}




\begin{tabular}{|c|c|c|c|c|c|c|c|c|c|c|c|c|c|c|c|c|c|c|c|c|c|c|c|}
\hline Guespalapa & $\begin{array}{l}\text { El Hoyo crater } \\
\text { vesicular lava }\end{array}$ & CH-07-14 & $99^{\circ} 10^{\prime} 02^{\prime \prime}$ & $19^{\circ} 05^{\prime} 25^{\prime \prime}$ & WSU/CGEO & Andesitic & Calc-alk. & * & $\begin{array}{l}\text { Straub et al. } \\
\text { (2011); this } \\
\text { study }\end{array}$ & 54.50 & 0.95 & 16.76 & 7.63 & 0.12 & 7.79 & 7.79 & 3.62 & 0.86 & 0.16 & & 99.42 & 69.9 & 1.0 \\
\hline Suchiooc & Suchiooc Cone lava & CH-05-2 & $99^{\circ} 06^{\prime} 16^{\prime \prime}$ & $19^{\circ} 04^{\prime} 06^{\prime \prime}$ & WSU/CGEO & Basaltic & Calc-alk. & & This study & 53.67 & 1.28 & 16.10 & 8.21 & 0.13 & 8.48 & 7.92 & 3.76 & 1.11 & 0.29 & 0.21 & 100.13 & 70.1 & \\
\hline Suchiooc & Suchiooc Cone bomb & CH-08-8 & $99^{\circ} 06^{\prime} 18^{\prime \prime}$ & $19^{\circ} 04^{\prime} 03^{\prime \prime}$ & WSU/CGEO & Basaltic & Calc-alk. & * & $\begin{array}{l}\text { Straub et al. } \\
\text { (2011); this } \\
\text { study }\end{array}$ & 52.97 & 1.25 & 15.94 & 8.20 & 0.13 & 8.83 & 7.83 & 3.68 & 1.03 & 0.28 & & 99.31 & 71.0 & \\
\hline Suchiooc & Suchiooc Cone bomb & CH-08-7 & $99^{\circ} 06^{\prime} 18^{\prime \prime}$ & $19^{\circ} 04^{\prime} 03^{\prime \prime}$ & WSU/CGEO & Basaltic & High-Nb & & This study & 53.68 & 1.46 & 16.35 & 8.32 & 0.13 & 7.93 & 7.11 & 4.09 & 1.22 & 0.34 & & 99.80 & 68.4 & \\
\hline Suchiooc & Suchiooc distal lavas & CH-08-11 & $99^{\circ} 03^{\prime} 58^{\prime \prime}$ & $18^{\circ} 57^{\prime} 52^{\prime \prime}$ & WSU/CGEO & Basaltic & $\mathrm{High}-\mathrm{Nb}$ & * & $\begin{array}{l}\text { Straub et al. } \\
\text { (2011); this } \\
\text { study }\end{array}$ & 51.60 & 1.78 & 16.80 & 9.21 & 0.15 & 7.60 & 7.93 & 4.15 & 1.25 & 0.43 & & 99.99 & 65.3 & \\
\hline Suchiooc & Suchiooc distal lavas & CH-08-10 & $99^{\circ} 05^{\prime} 20^{\prime \prime}$ & $19^{\circ} 01^{\prime} 02^{\prime \prime}$ & WSU/CGEO & Basaltic & High-Nb & & This study & 51.70 & 1.70 & 16.89 & 8.99 & 0.14 & 7.74 & 7.79 & 3.99 & 1.14 & 0.42 & & 99.59 & 66.2 & \\
\hline Suchiooc & Suchiooc distal lavas & CH-08-9 & $99^{\circ} 05^{\prime} 20^{\prime \prime}$ & $19^{\circ} 01^{\prime} 02^{\prime \prime}$ & WSU/CGEO & Basaltic & High-Nb & & This study & 52.03 & 1.69 & 16.58 & 9.13 & 0.14 & 7.83 & 7.69 & 4.06 & 1.21 & 0.42 & & 99.87 & 66.1 & \\
\hline Suchiooc & Suchiooc distal lavas & CH-08-12 & $99^{\circ} 06^{\prime} 24^{\prime \prime}$ & $18^{\circ} 58^{\prime} 55^{\prime \prime}$ & WSU/CGEO & Basaltic & High-Nb & & This study & 52.17 & 1.78 & 16.52 & 9.40 & 0.15 & 7.18 & 7.51 & 3.87 & 1.35 & 0.52 & & 99.52 & 63.5 & \\
\hline Suchiooc & $\begin{array}{l}\text { Suchiooc proximal } \\
\text { lava }\end{array}$ & CH-08-6 $6^{\mathbf{h}}$ & $99^{\circ} 06^{\prime} 01^{\prime \prime}$ & $19^{\circ} 03^{\prime} 20^{\prime \prime}$ & WSU/CGEO & Basaltic & Calc-alk. & * & $\begin{array}{l}\text { Straub et al. } \\
\text { (2011); this } \\
\text { study }\end{array}$ & 53.23 & 1.26 & 16.11 & 8.26 & 0.13 & 9.18 & 7.70 & 3.73 & 1.04 & 0.28 & & 100.10 & 71.7 & \\
\hline Suchiooc & $\begin{array}{l}\text { Suchiooc proximal } \\
\text { lava }\end{array}$ & CH-08-13 & $99^{\circ} 06^{\prime} 18^{\prime \prime}$ & $19^{\circ} 04^{\prime} 06^{\prime \prime}$ & WSU/CGEO & Basaltic & Calc-alk. & & This study & 53.33 & 1.26 & 15.89 & 8.26 & 0.13 & 9.02 & 7.77 & 3.69 & 1.05 & 0.28 & & 99.87 & 71.3 & \\
\hline Suchiooc & $\begin{array}{c}\text { Suchiooc proximal } \\
\text { lava }\end{array}$ & CH-08-14 & $99^{\circ} 05^{\prime} 58^{\prime \prime}$ & $19^{\circ} 04^{\prime} 48^{\prime \prime}$ & WSU/CGEO & Basaltic & Calc-alk. & & This study & 53.12 & 1.25 & 15.74 & 8.22 & 0.13 & 9.27 & 7.67 & 3.57 & 1.21 & 0.29 & & 99.64 & 72.0 & \\
\hline Suchiooc & $\begin{array}{l}\text { Suchiooc proximal } \\
\text { lava }\end{array}$ & CH-07-1 & $99^{\circ} 06^{\prime} 02^{\prime \prime}$ & $19^{\circ} 03^{\prime} 33^{\prime \prime}$ & WSU/CGEO & Basaltic & Calc-alk. & & This study & 54.17 & 1.33 & 16.16 & 8.17 & 0.13 & 8.75 & 7.59 & 3.82 & 1.07 & 0.30 & & 100.68 & 70.9 & \\
\hline Suchiooc & Suchiooc proximal & CH-05-1 & $99^{\circ} 08^{\prime} 59^{\prime \prime}$ & $19^{\circ} 03^{\prime} 58^{\prime \prime}$ & WSU/CGEO & Basaltic & High-Nb & & This study & 53.07 & 1.59 & 16.61 & 8.62 & 0.14 & 7.12 & 7.59 & 4.10 & 1.24 & 0.41 & 0.17 & 99.63 & 65.3 & \\
\hline Suchiooc & $\begin{array}{l}\text { Suchiooc proximal } \\
\text { lava }\end{array}$ & CH-07-2 & $99^{\circ} 06^{\prime} 00^{\prime \prime}$ & $19^{\circ} 03^{\prime} 26^{\prime \prime}$ & WSU/CGEO & Basaltic & High-Nb & & This study & 52.17 & 1.67 & 16.51 & 8.84 & 0.14 & 7.70 & 7.62 & 4.07 & 1.21 & 0.41 & & 99.46 & 66.4 & \\
\hline Tuxtepec & Tuxtepec bomb & $\mathrm{S} 15^{\mathrm{i}}$ & $99^{\circ} 16^{\prime} 51^{\prime \prime}$ & $19^{\circ} 07^{\prime} 41^{\prime \prime}$ & Harvard & Basaltic & Calc-alk. & * & $\begin{array}{l}\text { Straub et al. } \\
\quad \text { (2011); this } \\
\text { study }\end{array}$ & 50.16 & 1.00 & 14.87 & 8.76 & 0.14 & 9.73 & 9.80 & 3.20 & 1.52 & 0.51 & 0.00 & 99.71 & 71.6 & \\
\hline Tuxtepec & Tuxtepec bomb & S14 & $99^{\circ} 16^{\prime} 51^{\prime \prime}$ & $19^{\circ} 07^{\prime} 41^{\prime \prime}$ & Harvard & Basaltic & Calc-alk. & & $\begin{array}{l}\text { study } \\
\text { This study }\end{array}$ & 49.91 & 0.98 & 14.66 & 8.75 & 0.14 & 10.02 & 9.62 & 3.15 & 1.55 & 0.51 & 0.01 & 99.30 & 72.3 & \\
\hline Yecahuazac & $\begin{array}{l}\text { Yecahuazac Cone } \\
\text { bomb }\end{array}$ & CH-08-15 & $99^{\circ} 05^{\prime} 41^{\prime \prime}$ & $19^{\circ} 04^{\prime} 26^{\prime \prime}$ & WSU/CGEO & Basaltic & Calc-alk. & * & $\begin{array}{l}\text { Straub et al. } \\
\text { (2011); this } \\
\text { study }\end{array}$ & 53.13 & 1.14 & 16.03 & 7.92 & 0.13 & 8.05 & 8.17 & 3.67 & 1.45 & 0.39 & & 99.29 & 69.8 & \\
\hline Yecahuazac & Yecahuazac lava flow & CH-08-16 & $99^{\circ} 05^{\prime} 46^{\prime \prime}$ & $19^{\circ} 05^{\prime} 46^{\prime \prime}$ & WSU/CGEO & Basaltic & High-Nb & & This study & 53.24 & 1.50 & 16.53 & 8.26 & 0.13 & 7.54 & 7.21 & 4.11 & 1.20 & 0.37 & & 99.27 & 67.5 & \\
\hline Yecahuazac & $\begin{array}{l}\text { Yecahuazac lava } \\
\text { flow? }\end{array}$ & MCH-06-6 ${ }^{\mathrm{j}}$ & $99^{\circ} 04^{\prime} 4$ & $19^{\circ} 05^{\prime} 20^{\prime \prime}$ & WSU/CGEO & (Basaltic) & High-Nb & & This study & & 1.41 & & & & & & & & 0.37 & & & & \\
\hline Yecahuazac & $\begin{array}{l}\text { Hecahuazac lava } \\
\text { flow? }\end{array}$ & CH-05-3 ${ }^{\mathbf{j}}$ & $99^{\circ} 05^{\prime} 43^{\prime \prime}$ & $19^{\circ} 05^{\prime} 05^{\prime \prime}$ & WSU/CGEO & (Basaltic) & High-Nb & & This study & & 1.50 & & & & & & & & 0.40 & & & & \\
\hline C. Magdalena & Shallow intrusive & CH-05-19 & $99^{\circ} 11^{\prime} 0$ & & & & & & & & 0.55 & & & & & & & & 0.17 & & & & \\
\hline $\begin{array}{l}\text { Cuatzin } \\
\text { late }\end{array}$ & Cuatzi & CH-09-16 & $99^{\circ} \mathrm{C}$ ( & & WSU/C & Andesi & Calc-alk. & & & 62.76 & 0.78 & 16.09 & 5.12 & 0.09 & 2.67 & 4.65 & 4.18 & 1.87 & 0.24 & & 97.95 & 54.3 & 16.5 \\
\hline Ocusacayo & tal lava & CH-05-6 & $99^{\circ} \mathrm{C}-2$. & $19^{\circ} 07^{\prime} 25^{\prime \prime}$ & WSU/CGEO & Andesi & Calc-alk. & & & 64.45 & 0.71 & 16.11 & 4.71 & 0.08 & 2.85 & 4.63 & 4.29 & 2.01 & 0.24 & & 99.61 & 57.9 & 16.8 \\
\hline Ocusacayo & & CH-05 & & & & & & & & & & & & & & & & & & & & & n.a. \\
\hline Pelado & & CH-O & & & GEO & Ande & Calc & & & 61.95 & 0.94 & 15.83 & 5.70 & 0.10 & 4.40 & 5.35 & 4.14 & 1.79 & 0.28 & & 99.91 & 63.7 & 12.5 \\
\hline Pelac & & & & & & & & & & & 1.14 & & & & & & & & 0.34 & & & & n.a. \\
\hline Pelado & Pelado & CH-07 & & & Ws & Andesitic & Calc- & & & 60.24 & 1.01 & 16.05 & 6.08 & 0.10 & 4.36 & 5.67 & 4.07 & 1.73 & 0.28 & & 98.98 & 62.0 & 10.8 \\
\hline Teuthli & & $\mathrm{CH}-0$ & & & & & & & & & 0.95 & & & & & & & & 0.21 & & & & n.a. \\
\hline Teuthli & 1 lava & $\mathrm{CH}-\mathrm{O}$ & 9 & $g^{\prime \prime}$ & $\mathrm{J} / \mathrm{CGEO}$ & (Andesitic) & $\mathrm{C}$ & & & & 1. & & & & & & & & 0.21 & & & & n.a. \\
\hline Tlaloc & & $\mathrm{CH}-\mathbf{0}$ & & & & & & & & & & & & & & & & & & & & & $\begin{array}{l}\text { n.a. } \\
\text { na. }\end{array}$ \\
\hline Tlaloc & & CH-05-5 & & & & & & & & & 0. & & & & & & & & 0.22 & & & & n.a. \\
\hline $\begin{array}{c}\text { Zempoala } \\
\text { Ridge }\end{array}$ & Shallow intrusive & CH-05-18 & $99^{\circ} 19^{\prime} 19^{\prime \prime}$ & $19^{\circ} 04^{\prime} 44^{\prime \prime}$ & WSU/CGEO & (Andesitic) & Calc-alk. & & This study & & $\begin{array}{l}0.00 \\
0.74\end{array}$ & & & & & & & & 0.19 & & & & $\begin{array}{l}\text { 11.a. } \\
\text { n.a. }\end{array}$ \\
\hline Zempoala & Shallow intrusive & CH-05-17 & $99^{\circ} 18^{\prime} 42^{\prime \prime}$ & $19^{\circ} 05^{\prime} 07^{\prime \prime}$ & WSU/CGEO & (Andesitic) & Calc-alk. & & This study & & 0.62 & & & & & & & & 0.18 & & & & n.a. \\
\hline $\begin{array}{l}\text { flow near } \\
\text { Tuxtepec }\end{array}$ & Lava flow & S13 & $99^{\circ} 16^{\prime} 30^{\prime \prime}$ & $19^{\circ} 07^{\prime} 16^{\prime \prime}$ & Harvard & Andesitic & Calc-alk. & & This study & 53.32 & 1.36 & 16.60 & 9.94 & 0.15 & 5.44 & 7.35 & 3.59 & 1.05 & 0.38 & 0.01 & 99.20 & 55.5 & 2.9 \\
\hline Muneco & Lava flow & S16 & $99^{\circ} 19^{\prime} 45^{\prime \prime}$ & $19^{\circ} 09^{\prime} 26^{\prime \prime}$ & Harvard & Andesitic & Calc-alk. & & This study & 60.14 & 0.81 & 17.09 & 5.83 & 0.10 & 4.07 & 5.48 & 4.21 & 1.65 & 0.23 & 0.01 & 99.61 & 61.4 & 10.2 \\
\hline
\end{tabular}


Table 1. Major and trace element data of calc-alkaline and high-Nb magmas of the central Mexican Volcanic Belt (Continued)

\begin{tabular}{|c|c|c|c|c|c|c|c|c|c|c|c|c|c|c|c|c|c|c|c|c|c|c|c|c|}
\hline Volcano & Stratigraphic Unit & Sample_ID & Oliv $^{\mathrm{g}}$ & $\mathrm{Neph}^{\mathrm{g}}$ & $\mathrm{Ni}$ & $\mathrm{Sc}$ & $\mathrm{V}$ & $\mathrm{Cr}$ & Co & B & $\mathrm{Li}$ & $\mathrm{Be}$ & Cs & $\mathrm{Ba}$ & $\mathrm{U}$ & $\mathrm{Tl}$ & $\mathrm{Rb}$ & $\mathrm{w}$ & Th & $\mathrm{Nb}$ & $\mathrm{Ta}$ & $\mathrm{La}$ & $\mathrm{Ce}$ & $\mathrm{Pb}$ \\
\hline Popocatepetl & $\begin{array}{l}\text { Ventorillo aphyric } \\
\text { dacite }\end{array}$ & СH-09-18 & & & 63 & 12.31 & 94.73 & 119.90 & 15.65 & & 24.68 & 1.80 & 3.32 & 500 & 2.13 & 0.37 & 59.87 & 0.39 & 5.94 & 6.84 & 0.50 & 20.30 & 43.95 & 9.87 \\
\hline Popocatepetl & $\begin{array}{l}\text { Dyke in upper } \\
\text { B.Nexpayantla }\end{array}$ & CH-09-19 & & & 17 & 5.76 & 52.56 & 37.65 & 7.54 & 10 & 21.11 & 1.62 & 2.06 & 520 & 1.81 & 0.33 & 51.34 & 0.20 & 4.57 & 5.72 & 0.43 & 16.05 & 33.06 & 9.43 \\
\hline Popocatepetl & $\begin{array}{l}\text { Volcaniclast in upper } \\
\text { B.Nexpayantla }\end{array}$ & СH-09-20 & & & 39 & 11.83 & 85.10 & 72.88 & 12.94 & & 24.63 & 1.56 & 2.60 & 456 & 1.58 & 0.41 & 49.00 & 0.26 & 4.25 & 5.26 & 0.38 & 15.92 & 33.49 & 8.90 \\
\hline Popocatepetl & $\begin{array}{l}\text { Volcaniclast in upper } \\
\text { B.Nexpayantla }\end{array}$ & CH-09-21 & & & 77 & 14.25 & 107.96 & 162.87 & 18.35 & & 17.78 & 1.70 & 2.82 & 456 & 1.60 & 0.40 & 47.41 & 0.30 & 4.47 & 6.82 & 0.46 & 17.93 & 38.50 & 8.76 \\
\hline Popocatepetl & $\begin{array}{l}\text { Dyke in lower } \\
\text { B. Nexpayantla }\end{array}$ & CH-09-22 & & & 76 & 16.98 & 140.93 & 164.88 & 23.35 & & 12.71 & 1.37 & 0.53 & 352 & 0.91 & 0.15 & 28.37 & 0.18 & 2.58 & 5.45 & 0.34 & 14.43 & 32.35 & 4.86 \\
\hline $\begin{array}{l}\text { Popocatepetl } \\
\text { Cuatepel }\end{array}$ & $\begin{array}{l}\text { Nealtican flows } \\
\text { Cuatepel lova flow }\end{array}$ & $\begin{array}{l}\text { Popo5 } \\
\text { CH-08-26 }\end{array}$ & & & 53 & 16.00 & 113.32 & 125.30 & 17.22 & 16 & 23.97 & 1.69 & 2.64 & 428 & 1.60 & 0.36 & 51.78 & 0.33 & 4.58 & 9.08 & 0.60 & 17.46 & 37.95 & 8.07 \\
\hline Cuatepe & Cuatepetel Cone & CH-08 & & & & & & & & & & & & & & & & & & & & & & \\
\hline Guespalapa & $\begin{array}{l}\text { Guespalapa shield } \\
\text { lava }\end{array}$ & CH-09-1 & & & 103 & 17.40 & 117.17 & 147.88 & 26.43 & & 12.79 & 1.48 & 0.83 & 263 & 0.66 & 0.16 & 20.74 & 0.13 & 1.93 & 7.54 & 0.47 & 13.19 & 30.35 & 4.63 \\
\hline Guespalapa & $\begin{array}{l}\text { Guespalapa shield } \\
\text { lava }\end{array}$ & CH-07-8 & & & 126 & 19.45 & 129.89 & 279.94 & 29.03 & & 12.61 & 1.09 & 0.83 & 234 & 0.57 & 0.15 & 19.38 & 0.13 & 1.68 & 4.16 & 0.25 & 9.60 & 22.08 & 4.20 \\
\hline Guespalapa & $\begin{array}{l}\text { Guespalapa shield } \\
\text { lava }\end{array}$ & CH-09-2 & & & 100 & 17.61 & 120.62 & 155.40 & 26.39 & & 13.79 & 1.56 & 0.85 & 271 & 0.67 & 0.16 & 21.65 & 0.13 & 1.97 & 7.61 & 0.48 & 13.49 & 31.07 & 4.70 \\
\hline Guespalapa & $\begin{array}{l}\text { Guespalapa shield } \\
\text { lava }\end{array}$ & S12A & & & 109 & 20.13 & 133.95 & 199.34 & 28.19 & 7 & 13.03 & 1.49 & 0.94 & 284 & 0.70 & 0.18 & 22.98 & 0.15 & 2.06 & 7.96 & 0.50 & 14.26 & 32.40 & 5.14 \\
\hline Guespalapa & $\begin{array}{l}\text { Guespalapa shield } \\
\text { lava }\end{array}$ & CH-07-9 & & & 125 & 19.57 & 130.87 & 288.80 & 28.89 & & 12.00 & 1.08 & 0.83 & 240 & 0.57 & 0.31 & 19.48 & 0.13 & 1.68 & 4.08 & 0.26 & 9.55 & 22.47 & 4.08 \\
\hline Guespalapa & $\begin{array}{l}\text { Guespalapa shield } \\
\text { lava }\end{array}$ & CH-05-11 & & & 104 & 17.80 & 123.74 & 132.66 & 27.52 & & 12.20 & 1.59 & 0.87 & 274 & 0.70 & 0.15 & 21.57 & 0.16 & 2.08 & 8.82 & 0.53 & 14.32 & 34.08 & 4.81 \\
\hline Guespalapa & $\begin{array}{l}\text { Guespalapa shield } \\
\text { lava }\end{array}$ & CH-05-14 & & & 101 & 18.22 & 123.02 & 142.35 & 26.68 & & 13.52 & 1.45 & 0.89 & 277 & 0.68 & 0.17 & 21.52 & 0.14 & 2.02 & 7.58 & 0.46 & 13.46 & 31.52 & 4.81 \\
\hline Guespalapa & $\begin{array}{l}\text { Guespalapa shield } \\
\text { lava }\end{array}$ & CH-08-3 & & & 137 & 13.86 & 101.30 & 204.49 & 21.41 & 9 & 20.03 & 1.50 & 1.42 & 464 & 1.18 & 0.30 & 35.68 & 0.22 & 3.76 & 7.26 & 0.46 & 19.19 & 39.88 & 7.49 \\
\hline & El Caballito bomb & & 1.3 & & & & & & & & & & & & & & & & & & & & & 4.52 \\
\hline & & & 1.8 & & & & & & & & & & & & & & & & & & & & & \\
\hline & & & 1.3 & & 1 & & & & & & & & & & & & & & & & & & & 39 \\
\hline & & & & & 96 & & & & & & & & & & & & & & & & & & & \\
\hline & $\mathrm{N}$ & c & & & & & & & 26 & & & & & & & & & & & & & & & 4.44 \\
\hline & & & & & & & & & & & & & & & & & & & & & & & & 39 \\
\hline Guespalapa & $\begin{array}{l}\text { El Hoyo crater rim } \\
\text { lava }\end{array}$ & CH-07-11 & & & 159 & 21.60 & 142.99 & 268.42 & 31.96 & & 11.14 & 1.03 & 0.66 & 209 & 0.50 & 0.10 & 16.18 & 0.12 & 1.53 & 3.61 & 0.22 & 9.12 & 21.57 & 3.62 \\
\hline Guespalapa & $\begin{array}{l}\text { El Hoyo crater rim } \\
\text { lava }\end{array}$ & CH-07-12 & & & 164 & 21.64 & 142.23 & 310.74 & 32.56 & & 11.24 & 1.03 & 0.66 & 214 & 0.49 & 0.10 & 16.22 & 0.12 & 1.52 & 3.61 & 0.22 & 9.08 & 21.69 & 3.65 \\
\hline Guespalapa & $\begin{array}{l}\text { El Hoyo crater rim } \\
\text { lava }\end{array}$ & CH-07-13 & & & 167 & 21.50 & 141.91 & 378.26 & 32.52 & & 10.95 & 1.01 & 0.66 & 214 & 0.48 & 0.09 & 16.06 & 0.12 & 1.50 & 3.53 & 0.22 & 9.04 & 21.48 & 3.59 \\
\hline Guespalapa & $\begin{array}{l}\text { El Hoyo crater rim } \\
\text { lava }\end{array}$ & CH-07-10 & & & 159 & 21.50 & 139.52 & 284.48 & 31.98 & & 10.84 & 1.05 & 0.67 & 210 & 0.49 & 0.10 & 16.14 & 0.12 & 1.53 & 3.65 & 0.22 & 9.07 & 21.34 & 3.68 \\
\hline Guespalapa & $\begin{array}{l}\text { El Hoyo crater } \\
\text { vesicular lava }\end{array}$ & CH-07-14 & & & 165 & 21.16 & 138.61 & 292.87 & 32.38 & & 11.20 & 1.01 & 0.73 & 213 & 0.54 & 0.04 & 17.02 & 0.13 & 1.65 & 3.72 & 0.23 & 9.29 & 21.92 & 3.79 \\
\hline
\end{tabular}




\begin{tabular}{|c|c|c|c|c|c|c|c|c|c|c|c|c|c|c|c|c|c|c|c|c|c|c|c|c|}
\hline Suchiooc & Suchiooc Cone lava & CH-05-2 & 5.1 & & 158 & 21.25 & 153.40 & 287.84 & 35.48 & & 9.56 & 1.35 & 0.53 & 289 & 0.67 & 0.11 & 17.94 & 0.15 & 2.21 & 11.40 & 0.67 & 16.58 & 37.64 & 4.71 \\
\hline Suchiooc & Suchiooc Cone bomb & CH-08-8 & 6.1 & & 169 & 20.64 & 148.96 & 307.26 & 36.26 & 4 & 8.70 & 1.33 & 0.48 & 272 & 0.64 & 0.08 & 15.53 & 0.14 & 2.14 & 10.32 & 0.61 & 15.91 & 35.62 & 4.14 \\
\hline Suchiooc & Suchiooc Cone bomb & CH-08-7 & 5.7 & & 182 & 19.05 & 133.04 & 222.03 & 34.21 & 6 & 10.28 & 1.70 & 0.63 & 320.15 & 0.79 & 0.11 & 20.25 & 0.22 & 2.47 & 17.39 & 1.03 & 19.27 & 41.64 & 4.65 \\
\hline Suchiooc & Suchiooc distal lavas & CH-08-11 & 14.2 & & 123 & 21.54 & 152.12 & 231.80 & 35.55 & 4 & $\begin{array}{r}9.46 \\
9.46\end{array}$ & 1.79 & 0.29 & 273.53 & 0.70 & 0.05 & 16.31 & 0.23 & 2.46 & 24.37 & 1.43 & 21.08 & $\begin{array}{l}41.04 \\
45.61\end{array}$ & 3.64 \\
\hline Suchiooc & Suchiooc distal lavas & CH-08-10 & 11.2 & & 136 & 19.64 & 136.13 & 242.52 & 33.81 & 4 & 8.08 & 1.76 & 0.41 & 268.89 & 0.74 & 0.07 & 15.97 & 0.23 & 2.27 & 21.32 & 1.25 & 19.62 & 42.32 & 3.73 \\
\hline Suchiooc & Suchiooc distal lavas & CH-08-9 & 11.6 & & 143 & 20.39 & 143.34 & 249.05 & 35.36 & 4 & 8.62 & 1.79 & 0.44 & 271.10 & 0.76 & 0.07 & 18.03 & 0.26 & 2.37 & 22.14 & 1.31 & 19.16 & 41.44 & 3.75 \\
\hline Suchiooc & Suchiooc distal lavas & CH-08-12 & 6.9 & & 133 & 21.21 & 152.27 & 252.15 & 34.18 & 10 & 11.17 & 2.01 & 0.82 & 313.66 & 0.89 & 0.13 & 23.69 & 0.44 & 2.77 & 24.32 & 1.42 & 24.16 & 51.66 & 4.78 \\
\hline Suchiooc & $\begin{array}{l}\text { Suchiooc proximal } \\
\text { lava }\end{array}$ & CH-08-6 ${ }^{\mathrm{h}}$ & 7.5 & & 207 & 20.74 & 148.26 & 263.69 & 37.86 & 5 & 9.87 & 1.36 & 0.50 & 288 & 0.67 & 0.14 & 16.10 & 0.16 & 2.22 & 11.13 & 0.67 & 16.26 & 36.40 & 4.30 \\
\hline Suchiooc & $\begin{array}{l}\text { Suchiooc proximal } \\
\text { lava }\end{array}$ & CH-08-13 & 6.0 & & 185 & 20.43 & 146.65 & 287.56 & 37.06 & 4 & 9.40 & 1.37 & 0.53 & 281 & 0.65 & 0.92 & 16.61 & 0.15 & 2.15 & 10.81 & 0.65 & 16.06 & 35.91 & 4.21 \\
\hline Suchiooc & $\begin{array}{l}\text { Suchiooc proximal } \\
\text { lava }\end{array}$ & CH-08-14 & 6.7 & & 210 & 20.62 & 147.69 & 320.04 & 38.11 & 5 & 8.76 & 1.39 & 0.54 & 295 & 0.67 & 0.14 & 18.77 & 0.16 & 2.20 & 11.08 & 0.66 & 16.31 & 36.45 & 4.28 \\
\hline Suchiooc & $\begin{array}{l}\text { Suchiooc proximal } \\
\text { lava }\end{array}$ & CH-07-1 & 4.2 & & 197 & 20.29 & 140.28 & 294.60 & 36.23 & & 9.46 & 1.43 & 0.49 & 270 & 0.65 & 0.13 & 16.82 & 0.17 & 2.13 & 12.92 & 0.75 & 16.34 & 35.06 & 4.26 \\
\hline Suchiooc & $\begin{array}{l}\text { Suchiooc proximal } \\
\text { lava }\end{array}$ & CH-05-1 & 6.2 & & 133 & 20.14 & 140.13 & 184.42 & 33.14 & & 10.84 & 1.74 & 0.63 & 339.42 & 0.84 & 0.31 & 20.29 & 0.20 & 2.67 & 18.92 & 1.10 & 22.05 & 48.74 & 5.83 \\
\hline Suchiooc & $\begin{array}{l}\text { Suchiooc proximal } \\
\text { lava }\end{array}$ & CH-07-2 & 10.1 & & 149 & 20.34 & 141.65 & 252.40 & 35.03 & & 9.97 & 1.76 & 0.45 & 287.87 & 0.74 & 0.07 & 18.03 & 0.23 & 2.35 & 21.81 & 1.29 & 20.22 & 43.94 & 3.78 \\
\hline Tuxtepec & Tuxtepec bomb & $\mathrm{S} 15^{\mathrm{i}}$ & 17.7 & 0.7 & 220 & 27.11 & 187.72 & 494.14 & 40.23 & 2 & 13.04 & 1.46 & 0.35 & 1095 & 1.12 & 0.05 & 18.50 & 0.12 & 4.70 & 3.82 & 0.22 & 43.99 & 101.83 & 10.08 \\
\hline Tuxtepec & Tuxtepec bomb & S14 & 18.4 & 0.7 & 249 & 27.05 & 182.01 & 498.27 & 41.70 & 2 & 11.77 & 1.22 & 0.42 & 1054 & 0.87 & 0.63 & 21.44 & 0.10 & 4.55 & 3.71 & 0.21 & 42.81 & 101.94 & 10.28 \\
\hline Yecahuazac & $\begin{array}{l}\text { Yecahuazac Cone } \\
\text { bomb }\end{array}$ & CH-08-15 & 6.6 & & 168 & 20.94 & 154.49 & 280.84 & 32.06 & 4 & 10.18 & 1.61 & 0.68 & 637 & 1.35 & 0.17 & 24.66 & 0.16 & 4.03 & 8.44 & 0.48 & 31.16 & 68.96 & 7.34 \\
\hline Yecahuazac & Yecahuazac lava flow & CH-08-16 & 5.7 & & 161 & 19.09 & 134.25 & 229.52 & 33.28 & 6 & 10.19 & 1.74 & 0.56 & 306.81 & 0.81 & 0.12 & 19.24 & 0.22 & 2.53 & 18.08 & 1.07 & 19.72 & 42.64 & 4.64 \\
\hline Yecahuazac & $\begin{array}{l}\text { Yecahuazac lava } \\
\text { flow? }\end{array}$ & МCH-06-6 & n.a. & & 141 & 19.17 & 135.32 & 221.16 & 31.63 & & 10.94 & 1.62 & 0.62 & 309.90 & 0.78 & 0.11 & 20.36 & 0.19 & 2.43 & 16.77 & 0.97 & 18.76 & 40.93 & 4.99 \\
\hline Yecahuazac & $\begin{array}{l}\text { Yecahuazac lava } \\
\text { flow? }\end{array}$ & $\mathrm{CH}-05-3^{\mathrm{j}}$ & n.a. & & 142 & 19.59 & 135.85 & 213.48 & 32.27 & & 10.79 & 1.68 & 0.61 & 308.88 & 0.80 & 0.22 & 20.14 & 0.20 & 2.44 & 18.83 & 1.10 & 19.63 & 42.96 & 4.73 \\
\hline C. Magdalena & Shallow intrusive & CH-05-19 & & & 7 & 7.90 & & & & & 15.75 & 1.48 & 0.17 & & 0.67 & 0.25 & & & 4.45 & & & & & 11.27 \\
\hline Cuatzin & Cuatzin lava flow & CH-09-16 & & & 37 & 12.67 & 84.35 & 77.09 & 13.74 & & 26.88 & 1.84 & 2.21 & 574 & 1.63 & 0.40 & 50.62 & 0.24 & 4.78 & 9.11 & 0.59 & 23.50 & 47.26 & 9.67 \\
\hline Ocusacayo & tal lava & CH-05-6 & & & 34 & 11.85 & 82.59 & 84.06 & 12.11 & & 24.23 & 1.6 & 2.0 & 60 & 1.76 & 0.4 & 46.14 & 0.1 & 5.1 & 6.6 & & & 49. & 11.00 \\
\hline Ocusacayo & & CH-05-7 & & & 37 & 11.94 & 82.12 & 92.02 & 12.10 & & 24.64 & 1.6 & 2.1 & 58 & 1.7 & 0.43 & 48.03 & 0.19 & 5.1 & 6.58 & 5 & 23.1 & 47.32 & 10.98 \\
\hline Pelado & & CH-07-3 & & & 80 & 14.02 & 93.44 & 171.7 & 18.30 & & 19.83 & 1.6 & 1.5 & 53 & 1.2 & 0.2 & 41.23 & 0.2 & 4.1 & 10.78 & & 22. & & 8.39 \\
\hline Pelad & & ASC42 & & & 55 & 13.88 & 93. & 111.5 & 18.05 & 11 & 19.2 & 1.9 & 1.3 & 51 & 1.3 & 0. & 38. & 0. & 4.2. & 14. & & & & 8.24 \\
\hline $\begin{array}{l}\text { Pelado } \\
\text { Pelo }\end{array}$ & Pelad & CH-07-4 & & & 67 & 15.45 & 100.51 & 159.30 & 19.58 & & 18.32 & 1.65 & 1.4 & 49 & 1.17 & 0.2 & 37.2 & 0.23 & 3.7 & 11.5 & & 20.8 & & \\
\hline Teuthli & V.Te & CH-05-9 & & & 7 & 17.83 & 126. & 38.6 & 16.2 & & 14.5 & 1.3 & 1.8 & 37 & 1.1 & 0.2 r & 37. & & 3.6 & 5. & & & 34. & 7.14 \\
\hline Teut & & & & & 11 & 25. & & & 22. & & 13. & & 1.2 & & & & & & & & & & & 4.95 \\
\hline Tlaloc & V.Tlalo & CH-05-4 & & & 74 & 16.24 & 112.90 & 124.52 & 20.42 & & 17.28 & 1.3 & 1.3 & 43 & 0.9 & 0.26 & 31.82 & 0.26 & 3.21 & 6.34 & 0.40 & 17.04 & 36.31 & 7.62 \\
\hline $\begin{array}{l}\text { Tlaloc } \\
\text { Tlaloc }\end{array}$ & V.Tlaloc shield lava & CH-05-5 & & & $\begin{array}{l}14 \\
67\end{array}$ & $\begin{array}{l}10.24 \\
16.53\end{array}$ & $\begin{array}{l}112.90 \\
115.05\end{array}$ & $\begin{array}{l}124.02 \\
113.93\end{array}$ & 20.04 & & 16.75 & $\begin{array}{l}1.50 \\
1.40\end{array}$ & 1.30 & 430 & 0.96 & 0.25 & 30.89 & 0.26 & $\begin{array}{l}5.21 \\
3.17\end{array}$ & $\begin{array}{l}0.34 \\
6.41\end{array}$ & 0.40 & $\begin{array}{l}17.02 \\
17.02\end{array}$ & 36.40 & 7.43 \\
\hline $\begin{array}{c}\text { Zempoala } \\
\text { Ridge }\end{array}$ & Shallow intrusive & CH-05-18 & & & 17 & 14.51 & 99.25 & 46.19 & 13.77 & & 17.64 & 1.41 & 1.04 & 448 & 1.14 & 0.27 & 38.70 & 0.12 & 3.28 & 5.34 & 0.35 & 15.66 & 31.62 & 7.81 \\
\hline $\begin{array}{c}\text { Zempoala } \\
\text { Ridge }\end{array}$ & Shallow intrusive & CH-05-17 & & & 16 & 11.84 & 77.16 & 55.11 & 10.49 & & 18.12 & 1.52 & 1.13 & 493 & 1.28 & 0.33 & 42.37 & 0.14 & 4.09 & 5.58 & 0.37 & 17.69 & 35.01 & 13.44 \\
\hline $\begin{array}{l}\text { flow near } \\
\text { Tuxtepec }\end{array}$ & Lava flow & S13 & & & 47 & 24.51 & 174.93 & 158.58 & 29.94 & 20 & 16.15 & 1.70 & 1.31 & 311 & 0.88 & 0.20 & 25.73 & 0.36 & 2.69 & 13.25 & 0.77 & 19.89 & 43.97 & 5.29 \\
\hline Muneco & Lava flow & S16 & & & 52 & 15.92 & 110.98 & 112.75 & 17.71 & 9 & 18.16 & 1.47 & 1.52 & 429 & 1.07 & 0.28 & 33.67 & 0.16 & 3.09 & 6.54 & 0.43 & 18.18 & 35.66 & 7.25 \\
\hline
\end{tabular}

(Continued) 
Table 1. Major and trace element data of calc-alkaline and high-Nb magmas of the central Mexican Volcanic Belt (Continued)

\begin{tabular}{|c|c|c|c|c|c|c|c|c|c|c|c|c|c|c|c|c|c|c|c|c|c|c|c|c|c|c|}
\hline Volcano & Stratigraphic Unit & Sample_ID & $\operatorname{Pr}$ & Mo & $\mathrm{Sr}$ & $\mathrm{Nd}$ & $\mathrm{Sm}$ & $\mathrm{Hf}$ & $\mathrm{Zr}$ & $\mathrm{Eu}$ & $\mathrm{Sn}$ & $\mathrm{Sb}$ & $\mathrm{Gd}$ & $\mathrm{Tb}$ & Dy & $\mathrm{Y}$ & Ho & $\mathrm{Er}$ & $\mathrm{Yb}$ & $\mathrm{Lu}$ & ${ }^{87} \mathrm{Sr} /{ }^{86} \mathrm{Sr}$ & 2 STEM & ${ }^{143} \mathrm{Nd} /{ }^{144} \mathrm{Nd}$ & 2 STEM & eNd & \\
\hline Popocatepetl & $\begin{array}{l}\text { Ventorillo aphyric } \\
\text { dacite }\end{array}$ & СH-09-18 & 5.67 & 1.17 & 486 & 23.52 & 4.98 & 4.79 & 190 & 1.33 & 1.42 & 0.23 & 4.33 & 0.63 & 3.45 & 19.87 & 0.67 & 1.86 & 1.74 & 0.27 & & & & & & \\
\hline Popocatepetl & $\begin{array}{l}\text { Dyke in upper } \\
\text { B.Nexpayantla }\end{array}$ & CH-09-19 & 4.13 & 0.96 & 427 & 16.79 & 3.46 & 3.67 & 167 & 0.94 & 1.21 & 0.18 & 2.93 & 0.41 & 2.38 & 12.95 & 0.48 & 1.26 & 1.29 & 0.18 & 0.70458 & 9 & 0.51277 & 6 & 2.66 & \\
\hline Popocatepetl & $\begin{array}{l}\text { Volcaniclast in upper } \\
\text { B.Nexpayantla }\end{array}$ & CH-09-20 & 4.27 & 0.88 & 482 & 17.45 & 3.77 & 4.14 & 167 & 1.15 & 1.15 & 0.17 & 3.47 & 0.52 & 2.95 & 17.27 & 0.59 & 1.65 & 1.57 & 0.24 & 0.70429 & 8 & 0.51286 & 5 & 4.41 & \\
\hline Popocatepetl & $\begin{array}{l}\text { Volcaniclast in upper } \\
\text { B.Nexpayantla }\end{array}$ & CH-09-21 & 5.01 & 1.05 & 470 & 20.76 & 4.45 & 4.51 & 186 & 1.29 & 1.22 & 0.20 & 4.01 & 0.60 & 3.35 & 19.59 & 0.66 & 1.84 & 1.73 & 0.26 & & & & & & \\
\hline Popocatepetl & $\begin{array}{l}\text { Dyke in lower } \\
\text { B. Nexpayantla }\end{array}$ & СH-09-22 & 4.39 & 0.69 & 455 & 19.05 & 4.37 & 4.03 & 165 & 1.31 & 1.02 & 0.07 & 4.15 & 0.63 & 3.56 & 20.57 & 0.71 & 1.98 & 1.86 & 0.28 & & & & & & \\
\hline Popocatepetl & Nealtican flows & Popo5 & 5.04 & & 468 & 20.35 & 4.46 & 4.50 & 185 & 1.29 & 1.40 & 0.18 & 4.16 & 0.63 & 3.58 & 20.51 & 0.71 & 1.98 & 1.89 & 0.29 & & & & & & \\
\hline $\begin{array}{l}\text { Cuatepel } \\
\text { Cuatepel }\end{array}$ & $\begin{array}{l}\text { Cuatepel lava flow } \\
\text { Cuatepetel Cone }\end{array}$ & $\begin{array}{l}\text { CH-08-26 } \\
\text { CH-08-25 }\end{array}$ & & & & & & & & & & & & & & & & & & & & & & & & \\
\hline Guespalapa & $\begin{array}{l}\text { Guespalapa shield } \\
\text { lava }\end{array}$ & CH-09-1 & 4.08 & 0.82 & 477 & 17.64 & 4.13 & 4.06 & 182 & 1.37 & 1.11 & 0.10 & 4.09 & 0.63 & 3.64 & 20.73 & 0.72 & 1.98 & 1.85 & 0.28 & & & & & & ת \\
\hline Guespalapa & $\begin{array}{l}\text { Guespalapa shield } \\
\text { lava }\end{array}$ & CH-07-8 & 3.11 & 0.87 & 374 & 14.06 & 3.55 & 3.26 & 131 & 1.20 & 0.94 & 0.10 & 3.55 & 0.56 & 3.22 & 18.50 & 0.65 & 1.78 & 1.66 & 0.25 & 0.70419 & 7 & 0.51293 & 6 & 5.65 & 3 \\
\hline Guespalapa & $\begin{array}{l}\text { Guespalapa shield } \\
\text { lava }\end{array}$ & CH-09-2 & 4.17 & 0.84 & 488 & 18.11 & 4.24 & 4.15 & 186 & 1.41 & 1.14 & 0.10 & 4.19 & 0.65 & 3.71 & 21.24 & 0.74 & 2.03 & 1.89 & 0.29 & 0.70381 & 9 & 0.51294 & 5 & 5.82 & 先 \\
\hline Guespalapa & $\begin{array}{l}\text { Guespalapa shield } \\
\text { lava }\end{array}$ & S12A & 4.38 & & 510 & 19.06 & 4.51 & 4.34 & 188 & 1.48 & 1.27 & 0.10 & 4.39 & 0.68 & 3.93 & 22.27 & 0.79 & 2.15 & 1.99 & 0.31 & 0.70383 & 9 & 0.51294 & 6 & 5.85 & 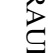 \\
\hline Guespalapa & $\begin{array}{l}\text { Guespalapa shield } \\
\text { lava }\end{array}$ & CH-07-9 & 3.13 & 0.85 & 373 & 14.11 & 3.49 & 3.28 & 131 & 1.17 & 0.89 & 0.10 & 3.59 & 0.56 & 3.25 & 18.69 & 0.66 & 1.80 & 1.64 & 0.25 & 0.70417 & 7 & 0.51292 & 6 & 5.54 & W \\
\hline Guespalapa & $\begin{array}{l}\text { Guespalapa shield } \\
\text { lava }\end{array}$ & СH-05-11 & 4.38 & 0.90 & 505 & 19.00 & 4.38 & 4.22 & 192 & 1.48 & 1.20 & 0.09 & 4.32 & 0.68 & 3.85 & 21.60 & 0.77 & 2.10 & 1.96 & 0.29 & 0.70376 & 7 & 0.51294 & 6 & 5.96 & $\overrightarrow{2}$ \\
\hline Guespalapa & $\begin{array}{l}\text { Guespalapa shield } \\
\text { lava }\end{array}$ & CH-05-14 & 4.12 & 0.86 & 484 & 18.24 & 4.18 & 4.07 & 185 & 1.42 & 1.15 & 0.09 & 4.19 & 0.65 & 3.74 & 20.82 & 0.75 & 2.03 & 1.91 & 0.28 & 0.70384 & 9 & 0.51294 & 5 & 5.85 & \\
\hline Guespalapa & $\begin{array}{l}\text { Guespalapa shield } \\
\text { lava }\end{array}$ & CH-08-3 & 5.08 & 1.06 & 456 & 20.69 & 4.29 & 4.47 & 186 & 1.27 & 1.14 & 0.14 & 3.86 & 0.58 & 3.29 & 19.18 & 0.65 & 1.83 & 1.74 & 0.27 & 0.70442 & 7 & 0.51282 & 4 & 3.61 & \\
\hline yespalapa & El Caballito bomb & TCu & & 1.03 & 511 & & & 4.42 & 206 & & 1.38 & 0.11 & 4.74 & 0.74 & 4.24 & & 0.85 & 2.33 & 2.17 & 0.33 & & 8 & 0.51292 & 11 & 5.43 & \\
\hline & omb & d & & 0. & 5 & $\begin{array}{l}21.0 \\
20.8\end{array}$ & & 4. & 21 & & 1. & 0.12 & 4. & 0.74 & $\begin{array}{l}4.27 \\
4.20\end{array}$ & 24.07 & 0.85 & 2.34 & 2.17 & 0.33 & 0.70363 & 6 & 0.51293 & 6 & 5.66 & \\
\hline & omb & & & $\begin{array}{l}1.02 \\
1.45\end{array}$ & $\begin{array}{l}529 \\
511\end{array}$ & $\begin{array}{l}20 \\
19\end{array}$ & & 4. & $\begin{array}{l}20 \\
19\end{array}$ & & $\begin{array}{l}1.34 \\
1.23\end{array}$ & $\begin{array}{l}0.10 \\
0.11\end{array}$ & $\begin{array}{l}4 . \\
4 .\end{array}$ & $\begin{array}{l}0.73 \\
0.69\end{array}$ & $\begin{array}{l}4.2 \\
3.9\end{array}$ & & $\begin{array}{l}0.84 \\
0.79\end{array}$ & 2. & $\begin{array}{l}16 \\
99\end{array}$ & $\begin{array}{l}0.32 \\
0.30\end{array}$ & 0.70366 & 6 & 0.51295 & 12 & 6.06 & \\
\hline & & & & 0.89 & 401 & 14. & & 3.48 & 1 & & 0.96 & 0.09 & 3.6 & 0.57 & 3.24 & 18.44 & 0.65 & 1. & 64 & 0.25 & & 8 & 0.51293 & 6 & 5.6 & \\
\hline & & & 3.3 & 0.94 & 403 & 14.7 & 3.5 & 3.5 & 14 & & 0.8 & 0.09 & 3.6 & 0.57 & 3.23 & & 0.64 & 1.73 & 1.61 & 0.25 & 0.70414 & 6 & 0.51292 & 9 & 5.59 & \\
\hline Guespalapa & $\begin{array}{l}\text { El Hoyo crater rim } \\
\text { lava }\end{array}$ & CH-07-11 & 3.04 & 0.80 & 362 & 13.82 & 3.52 & 3.10 & 123 & 1.14 & 0.83 & 0.09 & 3.64 & 0.57 & 3.40 & 19.76 & $\begin{array}{l}0.04 \\
0.69\end{array}$ & 1.92 & 1.80 & 0.27 & & & & & & \\
\hline Guespalapa & $\begin{array}{l}\text { El Hoyo crater rim } \\
\text { lava }\end{array}$ & CH-07-12 & 3.02 & 0.78 & 362 & 13.74 & 3.52 & 3.11 & 122 & 1.16 & 0.82 & 0.08 & 3.63 & 0.58 & 3.36 & 19.65 & 0.68 & 1.92 & 1.84 & 0.28 & 0.70411 & 9 & 0.51295 & 9 & 6.04 & \\
\hline Guespalapa & $\begin{array}{l}\text { El Hoyo crater rim } \\
\text { lava }\end{array}$ & CH-07-13 & 3.01 & 0.79 & 351 & 13.70 & 3.46 & 3.07 & 121 & 1.14 & 0.82 & 0.09 & 3.59 & 0.57 & 3.35 & 19.60 & 0.68 & 1.91 & 1.81 & 0.28 & & & & & & \\
\hline Guespalapa & $\begin{array}{l}\text { El Hoyo crater rim } \\
\text { lava }\end{array}$ & CH-07-10 & 3.00 & 0.78 & 360 & 13.92 & 3.57 & 3.12 & 122 & 1.18 & 0.82 & 0.08 & 3.62 & 0.57 & 3.30 & 19.39 & 0.68 & 1.94 & 1.83 & 0.28 & & & & & & \\
\hline Guespalapa & $\begin{array}{l}\text { El Hoyo crater } \\
\text { vesicular lava }\end{array}$ & CH-07-14 & 3.06 & 0.72 & 349 & 13.70 & 3.43 & 3.11 & 124 & 1.13 & 0.83 & 0.10 & 3.58 & 0.56 & 3.33 & 19.17 & 0.67 & 1.84 & 1.73 & 0.27 & 0.70418 & 6 & 0.51292 & 7 & 5.42 & \\
\hline & one laya & c & & 1.2 & 547 & 21.6 & 4. & 3. & 17 & & 1. & 0.07 & 4. & 0 . & 4. & & 0.84 & 2.32 & 2.15 & 0.32 & & 6 & & 5 & 6.42 & \\
\hline & & & & 0. & 52 & & & 3. & 161 & & 1. & & 4. & & & & & & & & & 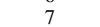 & & 5 & & \\
\hline & & & & 1. & 537 & & & & 206.44 & & & & & & 4. & & & & & & & 8 & & 7 & & \\
\hline Suchiooc & Suchiooc distal lavas & CH-08-11 & 5.80 & 1.20 & 548 & 24.51 & 5.49 & 4.78 & 229.52 & 1.80 & 1.44 & 0.06 & 5.51 & 0.86 & 5.02 & 28.39 & 1.00 & 2.73 & 2.51 & 0.38 & 0.70337 & 9 & 0.51294 & 6 & 5.93 & \\
\hline
\end{tabular}




\begin{tabular}{|c|c|c|c|c|c|c|c|c|c|c|c|c|c|c|c|c|c|c|c|c|c|c|c|c|c|}
\hline Suchiooc & Suchiooc distal lavas & CH-08-10 & 5.41 & 1.44 & 535 & 22.84 & 5.10 & 4.43 & 212.01 & 1.67 & 1.31 & 0.07 & 5.10 & 0.79 & 4.61 & 26.40 & 0.93 & 2.54 & 2.34 & 0.35 & 0.70342 & 8 & 0.51294 & 6 & 5.89 \\
\hline Suchiooc & Suchiooc distal lavas & CH-08-9 & 5.34 & 1.53 & 537 & 22.56 & 5.05 & 4.62 & 219.80 & 1.67 & 1.42 & 0.08 & 5.04 & 0.79 & 4.60 & 26.08 & 0.92 & 2.52 & 2.33 & 0.35 & & & & & \\
\hline Suchiooc & Suchiooc distal lavas & CH-08-12 & 6.65 & 1.62 & 500 & 28.07 & 6.12 & 5.31 & 262.32 & 1.93 & 1.48 & 0.13 & 6.03 & 0.92 & 5.39 & 30.75 & 1.08 & 2.94 & 2.71 & 0.41 & & & & & \\
\hline Suchiooc & $\begin{array}{l}\text { Suchiooc proximal } \\
\text { lava }\end{array}$ & CH-08-6 ${ }^{\mathrm{h}}$ & 4.87 & 1.00 & 532 & 20.96 & 4.73 & 3.79 & 172 & 1.49 & 1.08 & 0.05 & 4.56 & 0.70 & 4.04 & 23.30 & 0.81 & 2.23 & 2.08 & 0.32 & 0.70360 & 8 & 0.51294 & 6 & 5.93 \\
\hline Suchiooc & $\begin{array}{l}\text { Suchiooc proximal } \\
\text { lava }\end{array}$ & CH-08-13 & 4.80 & 1.01 & 524 & 20.63 & 4.66 & 3.70 & 164 & 1.45 & 1.10 & 0.06 & 4.51 & 0.69 & 3.98 & 22.98 & 0.80 & 2.20 & 2.05 & 0.31 & & & & & \\
\hline Suchiooc & $\begin{array}{l}\text { Suchiooc proximal } \\
\text { lava }\end{array}$ & CH-08-14 & 4.89 & 1.01 & 529 & 20.94 & 4.71 & 3.78 & 166 & 1.49 & 1.07 & 0.06 & 4.55 & 0.70 & 4.05 & 23.11 & 0.81 & 2.23 & 2.07 & 0.31 & & & & & \\
\hline Suchiooc & $\begin{array}{l}\text { Suchiooc proximal } \\
\text { lava }\end{array}$ & CH-07-1 & 4.71 & 1.43 & 507 & 20.60 & 4.68 & 3.85 & 169 & 1.50 & 1.13 & 0.06 & 4.55 & 0.71 & 3.95 & 22.88 & 0.80 & 2.27 & 2.08 & 0.32 & 0.70360 & 8 & 0.51294 & 6 & 5.93 \\
\hline Suchiooc & $\begin{array}{l}\text { Suchiooc proximal } \\
\text { lava }\end{array}$ & CH-05-1 & 6.37 & 1.80 & 579 & 26.65 & 5.94 & 4.78 & 225.32 & 1.82 & 1.41 & 0.09 & 5.57 & 0.86 & 4.91 & 27.72 & 0.97 & 2.65 & 2.46 & 0.37 & 0.70357 & 7 & 0.51294 & 6 & 5.99 \\
\hline Suchiooc & $\begin{array}{l}\text { Suchiooc proximal } \\
\text { lava }\end{array}$ & CH-07-2 & 5.61 & 1.97 & 541 & 23.99 & 5.41 & 4.63 & 218.07 & 1.74 & 1.38 & 0.07 & 5.35 & 0.83 & 4.80 & 27.50 & 0.96 & 2.69 & 2.43 & 0.37 & 0.70341 & 6 & 0.51292 & 8 & 5.56 \\
\hline Tuxtepec & Tuxtepec bomb & $\mathrm{S}^{5^{\mathrm{i}}}$ & 13.97 & & 938 & 61.26 & 12.32 & 3.94 & 160 & 3.15 & 1.01 & 0.04 & 9.19 & 1.17 & 5.36 & 28.56 & 0.98 & 2.56 & 2.15 & 0.32 & & & & & 6.87 \\
\hline Tuxtepec & Tuxtepec bomb & S14 & 13.62 & & 954 & 59.76 & 12.08 & 3.81 & 155 & 3.06 & 0.94 & 0.05 & 8.87 & 1.14 & 5.36 & 27.67 & 0.96 & 2.51 & 2.09 & 0.31 & & & & & \\
\hline Yecahuazac & $\begin{array}{l}\text { Yecahuazac Cone } \\
\text { bomb }\end{array}$ & CH-08-15 & 9.20 & 1.19 & 766 & 38.85 & 7.65 & 4.61 & 199 & 2.17 & 1.01 & 0.06 & 6.03 & 0.82 & 4.23 & 22.97 & 0.79 & 2.12 & 1.88 & 0.28 & 0.70430 & 8 & 0.51293 & 7 & 5.78 \\
\hline Yecahuazac & Yecahuazac lava flow & CH-08-16 & 5.51 & 1.41 & 539 & 23.27 & 5.18 & 4.52 & 211.23 & 1.66 & 1.30 & 0.08 & 5.06 & 0.78 & 4.51 & 25.74 & 0.90 & 2.47 & 2.28 & 0.34 & & & & & \\
\hline Yecahuazac & $\begin{array}{l}\text { Yecahuazac lava } \\
\text { flow? }\end{array}$ & МCH-06-6 & 5.42 & 1.64 & 536 & 22.49 & 5.11 & 4.41 & 200.82 & 1.59 & 1.28 & 0.08 & 4.87 & 0.76 & 4.42 & 25.08 & 0.88 & 2.40 & 2.22 & 0.34 & & & & & \\
\hline Yecahuazac & $\begin{array}{l}\text { Yecahuazac lava } \\
\text { flow? }\end{array}$ & CH-05- $3^{\mathrm{j}}$ & 5.58 & 1.80 & 537 & 23.27 & 5.28 & 4.54 & 212.59 & 1.65 & 1.35 & 0.08 & 5.06 & 0.79 & 4.60 & 25.95 & 0.91 & 2.51 & 2.30 & 0.35 & & & & & \\
\hline C.Magdalena & Shallow intrusive & CH-05-19 & 4.63 & 0.56 & 408 & 17.78 & 3.83 & 2.21 & 64 & 1.02 & 1.26 & 0.19 & 3.25 & 0.50 & 2.71 & 15.82 & 0.52 & 1.43 & 1.31 & 0.19 & & & & & \\
\hline Cuatzin & Cuatzin lava flow & CH-09-16 & 5.89 & 1.07 & 430 & 23.44 & 4.80 & 5.23 & 232 & 1.40 & 1.33 & 0.15 & 4.35 & 0.66 & 3.66 & 21.79 & 0.73 & 2.01 & 1.88 & 0.29 & 0.70450 & 7 & 0.51284 & 5 & 3.87 \\
\hline Ocusacayo & Ocusacayo distal lava & CH-05-6 & 6.58 & 1.54 & 499 & 25.13 & 5.26 & 5.12 & 209 & 1.40 & 1.26 & 0.20 & 4.22 & 0.64 & 3.46 & 19.20 & 0.66 & 1.79 & 1.66 & 0.25 & 0.70443 & 9 & 0.51290 & 5 & 5.07 \\
\hline Ocusacayo & va & CH-05-7 & 6.17 & 1.51 & 470 & 23.62 & 5.00 & 5.09 & 207 & 1.35 & 1.29 & 0.21 & 4.07 & 0.62 & 3.43 & 19.06 & 0.65 & 1.79 & 1.68 & 0.25 & & & & & \\
\hline $\begin{array}{l}\text { Pelado } \\
\text { Pas }\end{array}$ & Pelado Cone b & CH-07-3 & 5.66 & 1.58 & 460 & 22.95 & 4.78 & 5.02 & 212 & 1.36 & 1.23 & 0.12 & 4.44 & 0.67 & 3.65 & 21.11 & 0.71 & 2.00 & 1.88 & 0.29 & & & & & \\
\hline Pelado & Pelado shield lava & ASC42 & 6.21 & 1.43 & 497 & 25.04 & 5.19 & 5.35 & 241 & 1.54 & 1.42 & 0.11 & 4.77 & 0.72 & 4.07 & 23.50 & 0.81 & 2.22 & 2.07 & 0.31 & & & & & \\
\hline Pelado & d lava & CH-07-4 & 5.40 & 1.57 & 453 & 21.97 & 4.67 & 4.80 & 209 & 1.37 & 1.27 & 0.11 & 4.38 & 0.67 & 3.74 & 21.57 & 0.74 & 2.06 & 1.93 & 0.29 & & & & & \\
\hline Teuthli & & CH-05 & 4.65 & 0.93 & 534 & 18.91 & 4.32 & 4.15 & 171 & 1.29 & 1.20 & 0.17 & 3.99 & 0.63 & 3.66 & 20.98 & 0.73 & 2.04 & 1.93 & 0.29 & & & & & \\
\hline Teuthli & V.Teuthli shield lava & CH-05-8 & 4.19 & 0.78 & 433 & 18.07 & 4.53 & 3.96 & 169 & 1.41 & 1.35 & 0.10 & 4.70 & 0.78 & 4.75 & 27.60 & 0.97 & 2.73 & 2.62 & 0.40 & & & & & \\
\hline Tlaloc & V.Tlaloc shield lava & CH-05-4 & 4.74 & 1.17 & 451 & 19.06 & 4.30 & 4.40 & 179 & 1.28 & 1.14 & 0.15 & 3.98 & 0.63 & 3.62 & 21.18 & 0.72 & 2.03 & 1.94 & 0.29 & & & & & \\
\hline Tlaloc & eld lava & CH-05- & 4.80 & 1.15 & 452 & 19.15 & 4.34 & 4.37 & 181 & 1.29 & 1.14 & 0.14 & 4.00 & 0.64 & 3.68 & 21.23 & 0.73 & 2.04 & 1.95 & 0.29 & & & & & \\
\hline Zempoala Ridge & Shall & CH-05-18 & 4.29 & 1.15 & 422 & 16.86 & 3.88 & 4.31 & 170 & 1.12 & 1.07 & 0.13 & 3.49 & 0.55 & 3.16 & 18.26 & 0.61 & 1.70 & 1.59 & 0.24 & & & & & \\
\hline Zempoala Ridge & Shallow intrusive & CH-05-17 & 4.48 & 0.88 & 448 & 17.20 & 3.77 & 4.28 & 171 & 1.08 & 1.00 & 0.13 & 3.21 & 0.49 & 2.77 & 15.61 & 0.54 & 1.48 & 1.38 & 0.21 & 0.70422 & 9 & 0.51286 & 6 & 4.29 \\
\hline $\begin{array}{l}\text { flow near } \\
\text { Tuxtepec }\end{array}$ & Lava flow & S13 & 5.66 & & 350 & 24.17 & 5.52 & 4.78 & 218 & 1.70 & 1.87 & 0.07 & 5.88 & 0.95 & 5.76 & 33.85 & 1.20 & 3.33 & 3.13 & 0.48 & & & & & \\
\hline Muneco & Lava flow & S16 & 4.71 & & 482 & 19.51 & 4.25 & 4.37 & 180 & 1.29 & 1.04 & 0.12 & 3.96 & 0.60 & 3.36 & 20.42 & 0.68 & 1.89 & 1.76 & 0.27 & & & & & \\
\hline
\end{tabular}

Major element oxide abundances in wt\%. Trace element abundances in ppm. Total Fe given as $\mathrm{Fe}_{2} \mathrm{O}_{3}$

Run error of isotope ratios given as 2 standard errors of the mean in ppm.

${ }^{a}$ Indicates laboratory were major and trace elements were obtained (Harvard, Langmuir Laboratory; WSU, Washington State University; CGEO, Centro de Geociencias).

${ }^{\mathrm{b}}$ Andesitic (quartz-normative) and basaltic (olivine-normative). Inferred from petrograpy and stratigraphic context for samples without major element oxides.

${ }^{\mathrm{c}}$ Division in calc-alkaline $(<16 \mathrm{ppm} \mathrm{Nb})$ and high-Nb arc magmas $(>16 \mathrm{ppm} \mathrm{Nb})$.

${ }^{\mathrm{d} D e n o t e s ~ s a m p l e ~ f o r ~ w h i c h ~} 3 \mathrm{He} / 4 \mathrm{He}$ of olivines have been obtained (see Straub et al. 2011).

${ }^{\mathrm{e}} \mathrm{Selected}$ data $\left(\mathrm{SiO}_{2}, \mathrm{FeO} * \mathrm{MgO}, \mathrm{Ni}, \mathrm{Sr}, \mathrm{Nb}, \mathrm{La}, \mathrm{Gd}, \mathrm{Y}, \mathrm{Yb}, \mathrm{Nd}\right.$ isotope ratios, normative Qtz, normative Oliv) for some samples have previously been reported by Straub et al. (2011).

${ }^{\mathrm{f}}$ Bulk rock Mg\#, calculated assuming $18 \%$ ferric Fe.

${ }^{g}$ CIPW norm: Qtz, quartz-normative; Oliv, olivine normative. Calculation with program created by K. Hollocher at the Union College of Schenectady (http://www.union.edu/PUBLIC/GEODEPT/

COURSES/petrology/norms.htm)

${ }^{\mathrm{h}} \mathrm{Sr}$ and $\mathrm{Nd}$ isotope ratios of this sample are those of sample $\mathrm{CH}$ 07-1 that was taken at the same location.

${ }^{\mathrm{i}} \mathrm{Sr}$ and $\mathrm{Nd}$ isotopes are those of sample pw115 (LaGatta), a sample from same location with identical major and trace elements.

${ }^{\mathrm{j}}$ Origin from Yecahuazac Cone possible, but not certain. 
western flank of Yecahuazac cones actually derives from V. Suchiooc, and not from Yecahuazac as stated by Wallace \& Carmichael (1999) and LaGatta (2003).

Volcan Tuxtepec is a small scoria cone to the east of the village of Capulin (Wallace \& Carmichael 1999). It erupts olivine- and plagioclase-phyric olivine-normative calc-alkaline basalts with the lowest $\mathrm{SiO}_{2}=49.9-50.2 \mathrm{wt} \%$ and $\mathrm{Nb}=3.7-$ $3.8 \mathrm{ppm}$, but the highest $\mathrm{MgO}=9.7-10.0 \mathrm{wt} \%$ and $\mathrm{Mg \#}=71.6-72.3$ reported from the Sierra Chichinautzin Volcanic Field.

$V$. Popocatepetl is a major calc-alkaline MVB composite volcano that is well known for the presence of olivine phenocrysts in basaltic andesitic and andesitic magmas (e.g. Straub \& Martin-Del Pozzo 2001; LaGatta 2003; Schaaf et al. 2005). One quartz-normative basaltic andesite $\left(\mathrm{SiO}_{2}=\right.$ $56.7 \mathrm{wt} \%$, LaGatta 2003) from the oldest exposed series in Barranco Nexpayantla provided sufficient olivine for $\mathrm{He}$ isotope analyses (Straub et al.
2011). The most recent dating suggests a maximum age of $c .600 \mathrm{ka}$ for the oldest exposed Popo series (R. Espinasa-Pereña, work in progress).

\section{Analytical methods}

All new data are reported in Table 1. In addition, we report re-analyses of major element oxides by DCP of samples reported by Wallace \& Carmichael (1999) for which LaGatta (2003) had analysed for trace element and isotope ratios (see Supplementary material).

Bulk rock powders were analysed for major elements (50 samples) either at the Geoanalytical Laboratory of Washington State University, USA (XRF method, 44 samples), or at the Department of Earth and Planetary Sciences (Langmuir Laboratory) at Harvard University, USA (DCP method). Trace elements were determined by ICP-MS methods at the Centro de Geociencias (59 samples), Universidad Nacional Autónoma de
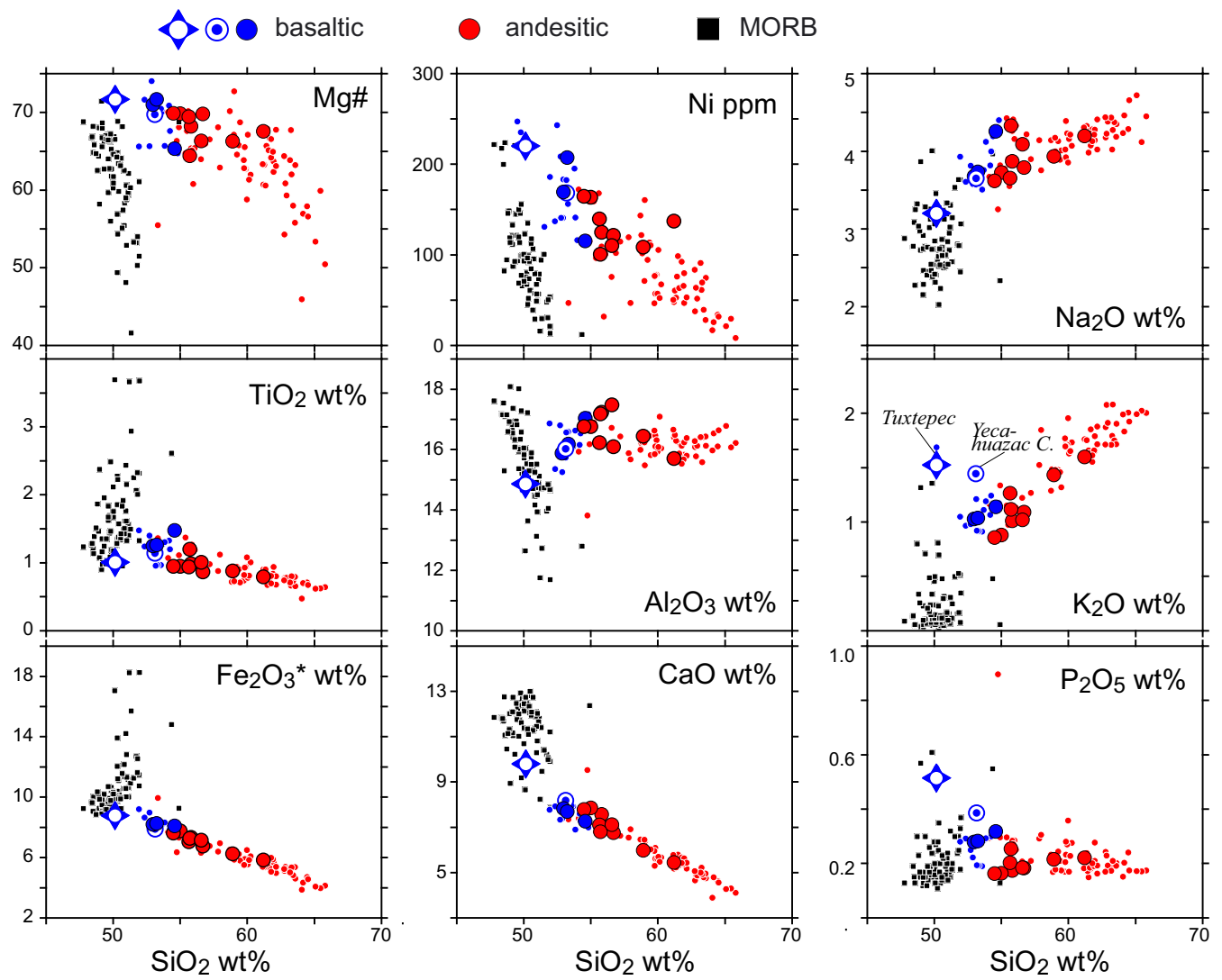

Fig. 3. Whole-rock Mg\# numbers and major element oxides v. $\mathrm{SiO}_{2}$ (in wt \%) of central MVB basaltic to andesitic magmas compared to East Pacific Rise MORB (filled squares) from Niu \& Batiza (1997). Mg\# number (=molar ratio of $\left.\left(\mathrm{Mg} /\left(\mathrm{Mg}+\mathrm{Fe}^{2+}\right)\right)\right)$ is calculated using $18 \%$ ferric iron after Straub et al. (2008). 
México, Querétaro, México or at Department of Earth and Planetary Sciences (Langmuir Laboratory) at Harvard University, USA. A selected subset of 29 samples was analysed for $\mathrm{Sr}$ and $\mathrm{Nd}$ isotope ratios by thermal ionization mass spectrometry at the Institute for Earth Sciences, Academia Sinica, Taipei, Taiwan. Details of sample preparation, analytical methods and assessment of interlaboratory comparability are given in the Supplementary material.

There is a small overlap with abundance data and ${ }^{143} \mathrm{Nd} /{ }^{144} \mathrm{Nd}$ ratios previously published by Straub et al. (2011). This is indicated in the footnote to Table 1. Olivine compositions and ${ }^{3} \mathrm{He} /{ }^{4} \mathrm{He}$ relevant to the discussion have been reported by Straub et al. $(2008,2011)$.

\section{Results}

\section{Major elements}

Major element oxides are presented in Figures 1 and 3 . The primitive nature of the calc-alkaline magmas is evident in the high $\mathrm{Ni}$ and $\mathrm{Mg \#}$ values that are comparable to mid-ocean ridge basalts (MORB), which best exemplify primitive melts from upper mantle peridotite. On the other hand, the central MVB magmas differ from MORB by the arc-typical enrichments in $\mathrm{SiO}_{2}, \mathrm{~K}_{2} \mathrm{O}$ and
$\mathrm{Na}_{2} \mathrm{O}$, and depletions of $\mathrm{CaO}$, and $\mathrm{Fe}_{2} \mathrm{O}_{3} *, \mathrm{MnO}$ (not shown) and $\mathrm{TiO}_{2}$, all of which are at the lower end, or below MORB. Only $\mathrm{Al}_{2} \mathrm{O}_{3}, \mathrm{P}_{2} \mathrm{O}_{5}$ and $\mathrm{MgO}$ (not shown) abundances compare with MORB. When plotted against $\mathrm{SiO}_{2}$, the major element oxides follow strikingly linear trends away from the field of MORB. Such trends are typical for melt mixing rather than for fractional crystallization (e.g. Reubi \& Blundy 2009). The linear trends are especially tight for major elements $\mathrm{TiO}_{2}, \mathrm{CaO}, \mathrm{Fe}_{2} \mathrm{O}_{3} *$ and $\mathrm{MgO}$ that are commonly thought to be mantle-derived in arc magmas. There is more variability of $\mathrm{K}_{2} \mathrm{O}, \mathrm{Na}_{2} \mathrm{O}$ and $\mathrm{P}_{2} \mathrm{O}_{5}$ that may be influenced by the slab flux (Straub et al. 2008, 2013). The basalts from Tuxtepec (S15) and Yecahuazac Cone (CH08-15), respectively, stand out because of their high $\mathrm{K}_{2} \mathrm{O}$ and $\mathrm{P}_{2} \mathrm{O}_{5}$ abundances.

\section{Incompatible trace elements}

Multi-element diagrams of incompatible elements reveal the arc-typical relative enrichments of Cs, $\mathrm{Ba}, \mathrm{U}, \mathrm{K}, \mathrm{Sr}$ and $\mathrm{Li}$ that are paired with relative depletions of $\mathrm{Nb}$, Ta and Ti (Fig. 4; only samples high $-{ }^{3} \mathrm{He} /{ }^{4} \mathrm{He}$ olivines are shown for clarity). A notable feature is that the andesites are more depleted and more homogeneous in incompatible elements than the basalts. The Guespalapa andesites

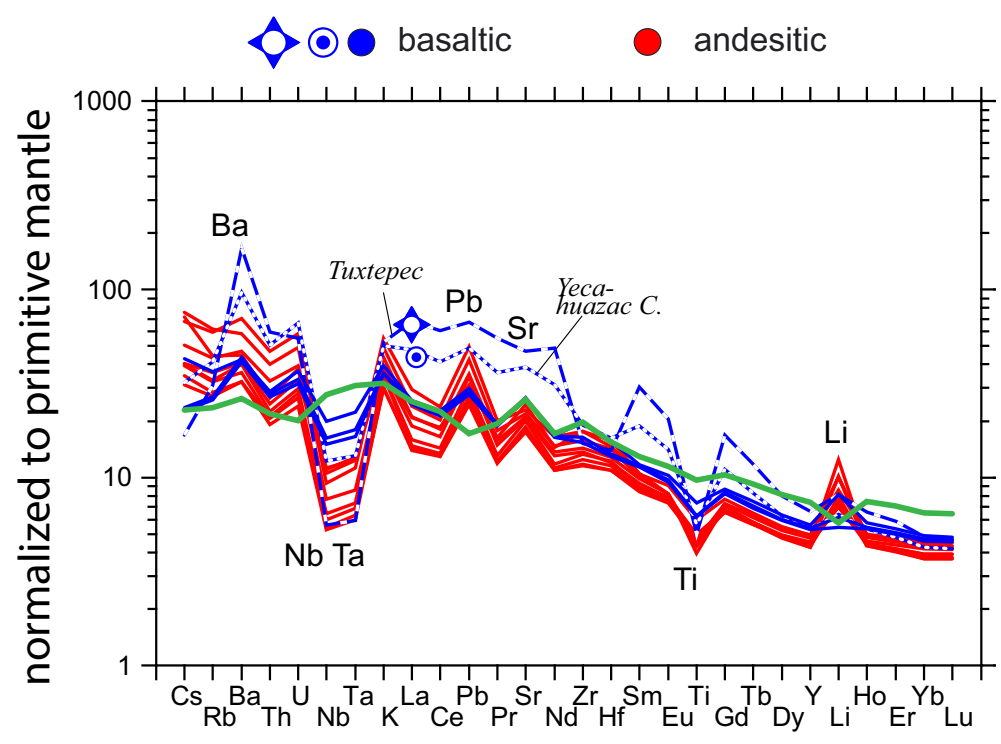

Fig. 4. Multi-element diagram of incompatible trace elements for whole-rock samples from the central MVB normalized to primitive mantle from McDonough \& Sun (1995). For clarity, only samples with high $-{ }^{3} \mathrm{He} /{ }^{4} \mathrm{He}$ olivines are shown. Blue lines show basaltic (olivine-normative) melts, with enriched high-La basalts Tuxtepec and Yecahuazac Cone being denoted by separate blue-white symbols; red lines andesitic (quartz-normative) melts. Old Texcal Flow magma (green line) is a high-Nb arc basalt of the Sierra Chichinautzin that is only minimally affected by slab additions and best represents a melt from background mantle prior to subduction processing (Straub et al. 2013). 
have the lowest abundances of light rare earth elements (LREE) and $\mathrm{Nb}$, while the basalts and, in particular those of Tuxtepec and Yecahuazac Cone, tend to be more enriched. Note that the Tuxtepec and Yecahuazac Cone basalts were targeted for the ${ }^{3} \mathrm{He} /{ }^{4} \mathrm{He}$ work because of their unusual trace element patterns, but are otherwise rare in the Sierra Chichinautzin.

The unusual composition of the Tuxtepec and Yecahuazac Cone basalts also shows in trends of individual trace elements v. $\mathrm{SiO}_{2}$ (Fig. 5). In general, the highly incompatible LILE (Cs, Ba, $\mathrm{Rb}, \mathrm{U}, \mathrm{Pb}, \mathrm{Th}, \mathrm{Pb}, \mathrm{Sr}$ ) increase with $\mathrm{SiO}_{2}$, while LREE and HFSE are largely indifferent towards $\mathrm{SiO}_{2}$. The Tuxtepec and Yecahuazac Cone basalts, however, are enriched in LREE and HFSE at low $\mathrm{SiO}_{2}$, except for $\mathrm{Nb}$ and $\mathrm{Ta}$, which are lower or comparable to the other calc-alkaline magmas. The heavy rare earth elements (HREE; Ho, Er,
$\mathrm{Yb}$ and $\mathrm{Lu}$ ) of all magmas always decrease slightly with $\mathrm{SiO}_{2}$ in tight trends that resemble those of the major element oxides $\mathrm{TiO}_{2}, \mathrm{CaO}$, $\mathrm{Fe}_{2} \mathrm{O}_{3}$ and $\mathrm{MgO}$.

Most central MVB magmas have fairly flat, HREE patterns with $\mathrm{Dy} / \mathrm{Yb}(=1.95 \pm 0.14$, $n=109)$ that are only slightly higher than MORB (Dy $/ \mathrm{Yb}=1.69 \pm 0.10, n=80$ ) and lack a recognizable 'garnet signature' (Fig. 6). Again, the exceptions are the Tuxtepec and Yecahuazac Cone basalts that have high $\mathrm{Dy} / \mathrm{Yb}$ of 2.5 and 2.3, respectively, which suggests a role of garnet in their genesis. Following Gomez-Tuena et al. (2007a), who report young MVB volcanic rocks with similar trace element signatures from the Valle de Bravo further to the west, Tuxtepec and Yecahuazac Cone basalts will be referred to as 'high-La basalts'. All other calc-alkaline basalts and andesites will be referred to as 'low-La magmas'.

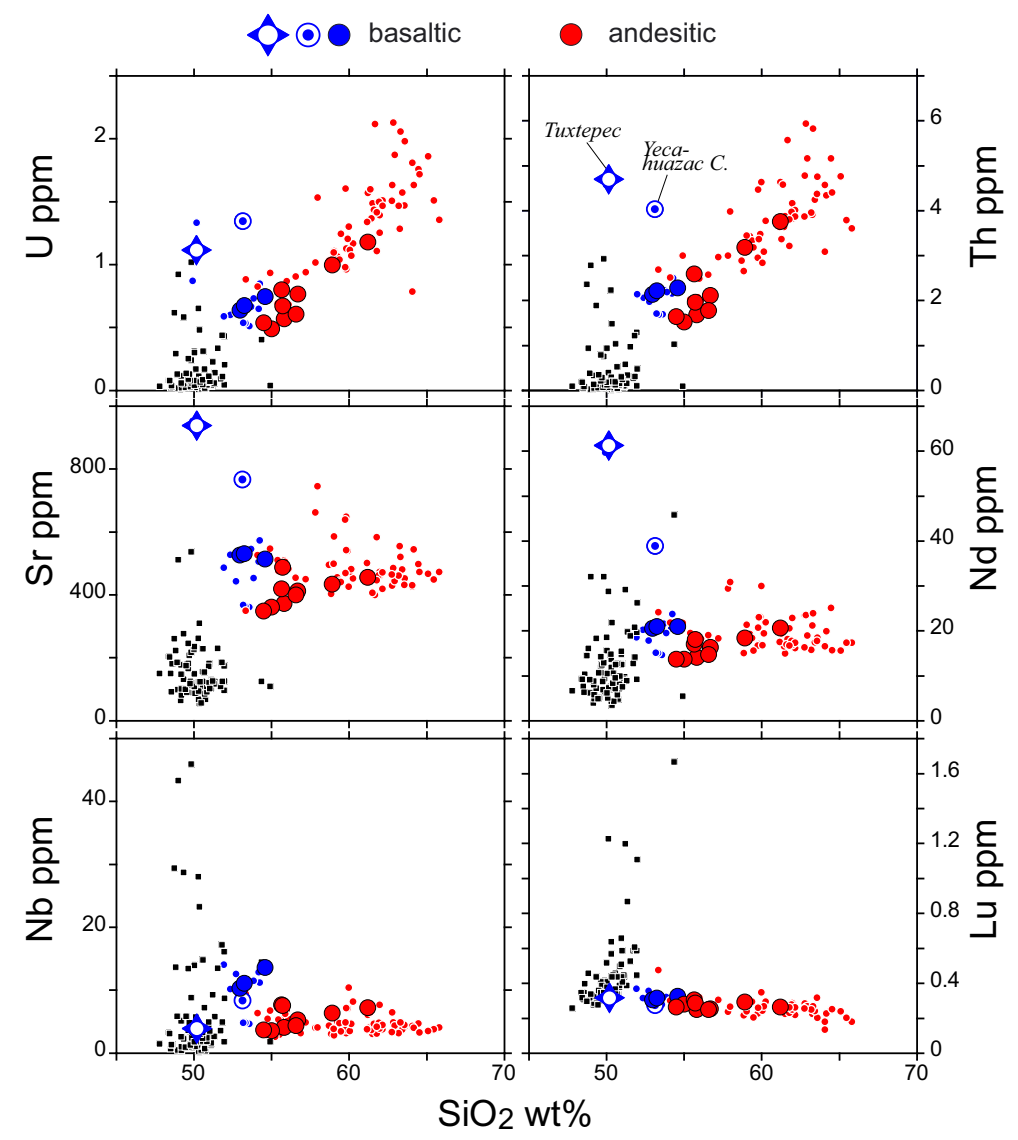

Fig. 5. Selected trace elements (in ppm) v. $\mathrm{SiO}_{2}(\mathrm{wt} \%$ ) of whole rocks of central MVB basaltic to andesitic magmas compared with East Pacific Rise MORB (filled squares) from Niu \& Batiza (1997). Note diverging behaviour of U and $\mathrm{Nb}$ despite similar incompatibility during melting and crystallization. 


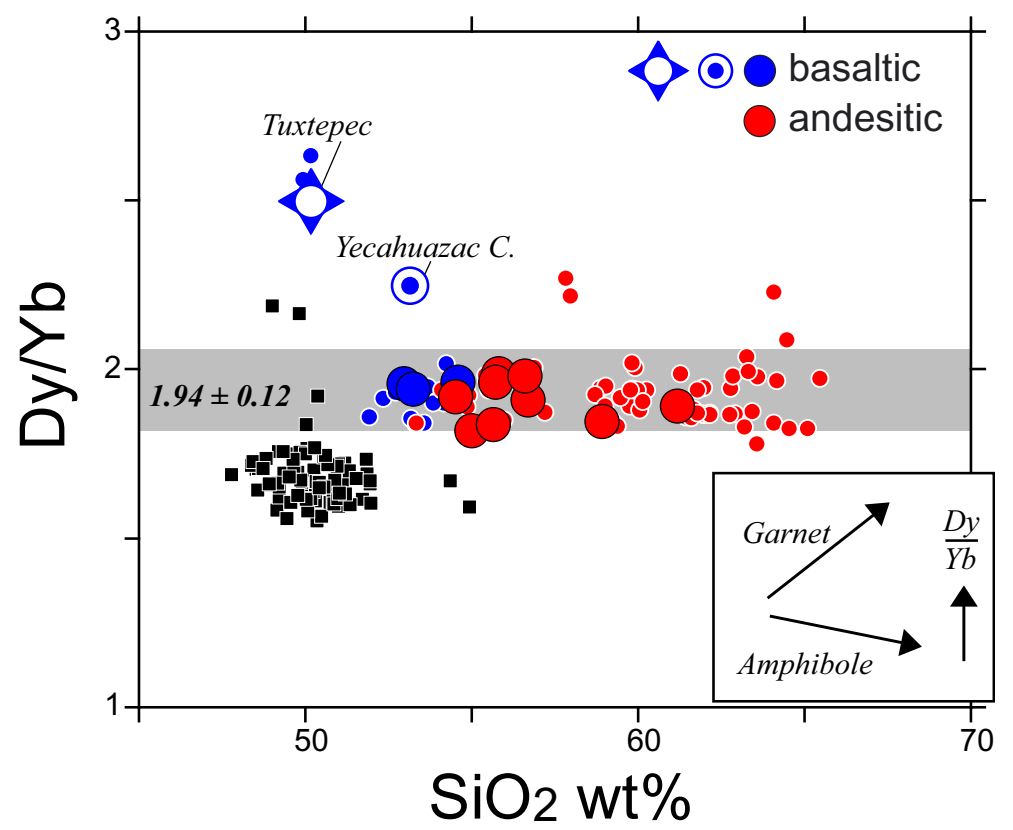

Fig. 6. Bulk rock $\mathrm{Dy} / \mathrm{Yb}$ v. $\mathrm{SiO}_{2}(\mathrm{wt} \%)$ in central MVB calc-alkaline basalts and andesites, compared with East Pacific Rise MORB from Niu \& Batiza (1997) (filled squares). Grey band is average of low-La basalts and andesite. Inset after Davidson et al. (2007) indicates the trends induced by fractional crystallization of amphibole and garnet.

\section{Sr and Nd isotope ratios}

It is no surprise that our new ${ }^{87} \mathrm{Sr} /{ }^{86} \mathrm{Sr}$ and ${ }^{143} \mathrm{Nd} /{ }^{144} \mathrm{Nd}$ data are similar to those reported previously from the central MVB (e.g. Siebe et al. 2004a; Schaaf et al. 2005; Straub et al. 2008). This means that the high- ${ }^{3} \mathrm{He} /{ }^{4} \mathrm{He}$ samples have fairly unradiogenic ${ }^{87} \mathrm{Sr} /{ }^{86} \mathrm{Sr}(=0.70350-0.70441)$ and radiogenic ${ }^{143} \mathrm{Nd} /{ }^{144} \mathrm{Nd}(=0.51282-0.51299)$, which are close to Pacific MORB and only marginally overlap with crustal compositions from within and around the MVB (Straub et al. 2008, 2013). ${ }^{87} \mathrm{Sr} /{ }^{86} \mathrm{Sr}$ and ${ }^{143} \mathrm{Nd} /{ }^{144} \mathrm{Nd}$ correlate broadly with $\mathrm{SiO}_{2}$, and andesites have systematically higher ${ }^{87} \mathrm{Sr} /{ }^{86} \mathrm{Sr}$ and lower ${ }^{143} \mathrm{Nd} /{ }^{144} \mathrm{Nd}$ than the basalts (Fig. 7). This shows that the basaltic and andesitic series cannot be related by simple fractional crystallization despite the coherent trends of major elements v. $\mathrm{SiO}_{2}$, and despite their proximity in time and space.

\section{Discussion}

The trace element and $\mathrm{Sr}-\mathrm{Nd}$ isotope flux in arcs

At first view, the trace elements of the calc-alkaline magmas display features that agree with the pyroxenite model proposed by Straub et al. (2011). For example, this model predicts correlations between slab-derived elements and melt $\mathrm{SiO}_{2}$, because high- $\mathrm{SiO}_{2}$ initial mantle melts are produced from silica-excess pyroxenites that contain higher amounts of the slab component. Figure 5 shows such correlations for highly incompatible elements (e.g. U, Th) that are generally slabcontrolled in arc magmas. For the same reason, and assuming the subducted sediment as source of the radiogenic $\mathrm{Sr}$ and unradiogenic Nd, the pyroxenite model predicts - as broadly observed - an increase in ${ }^{87} \mathrm{Sr} /{ }^{86} \mathrm{Sr}$ and a decrease in ${ }^{143} \mathrm{Nd} /{ }^{144} \mathrm{Nd}$ with increasing melt $\mathrm{SiO}_{2}$ (Fig. 7). The pyroxenite model can also account for the difference in trace element signatures between the low-La calcalkaline magmas and the high-La calc-alkaline basalts, as it proposes the flux of many individual slab components that may have very different trace element signatures and that infiltrate a heterogeneous subarc mantle (e.g. Straub et al. 2008; Tollstrup et al. 2010; Gomez-Tuena et al. 2011).

On the other hand, open questions remain. For example, the abundance data only point to a slab flux of the highly fluid-mobile LILE, but do not provide information about a slab flux of the middle rare earth elements (MREE) and HFSE. Moreover, it is not clear how the large amount of slab material required by the pyroxenite model fits 


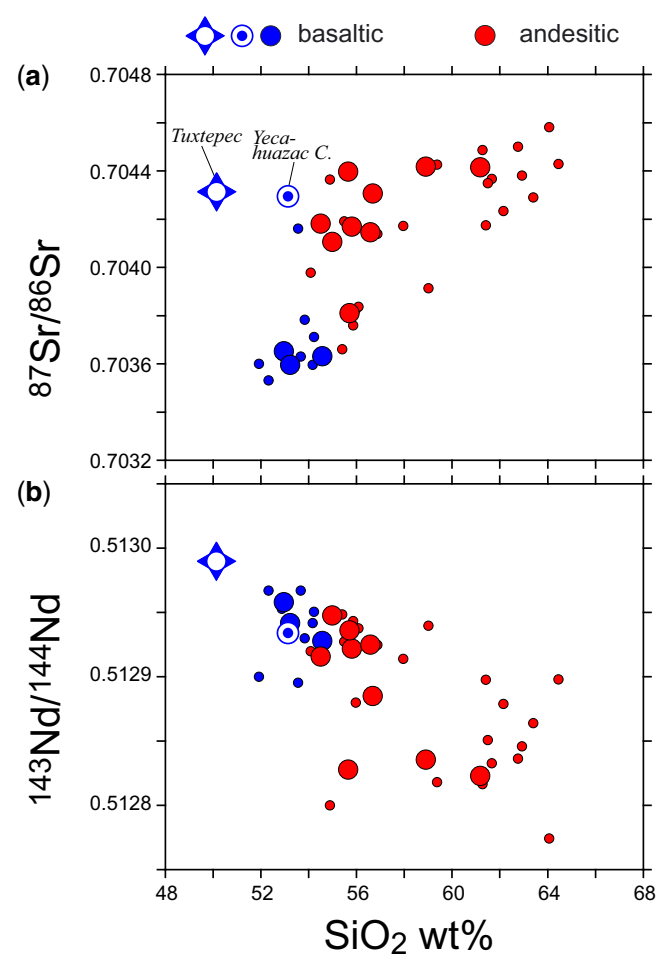

Fig. 7. Whole rock (a) ${ }^{87} \mathrm{Sr} /{ }^{86} \mathrm{Sr}$ and $(\mathbf{b}){ }^{143} \mathrm{Nd} /{ }^{144} \mathrm{Nd} \mathrm{v}$. melt $\mathrm{SiO}_{2}(\mathrm{wt} \%)$. Note systematic difference in basaltic and andesitic series which excludes a fractional crystallization link between the two series and implies source diversity.

with the flat HREE pattern of the magmas. The minimum amount of slab-derived $\mathrm{SiO}_{2}$ in the reaction pyroxenite is fixed by the stochiometric reaction that transforms mantle olivine to 'reaction orthopyroxene': $\mathrm{SiO}_{2}+(\mathrm{Mg}, \mathrm{Fe})_{2} \mathrm{SiO}_{4}=(\mathrm{Mg}, \mathrm{Fe})_{2}$ $\mathrm{Si}_{2} \mathrm{O}_{6}$. Assuming an original background mantle with $c$. 50-60\% olivine, the resulting reaction pyroxenite (which consists of c. 50-60\% reaction orthopyroxene, plus the original mantle ortho- and clinopyroxene) must contain at least c. $15-18 \%$ slab-derived material. The formation of silicaexcess pyroxenite requires even higher amounts (e.g. Straub et al. 2011). Given these amounts, why is there no garnet signature in the majority of the calc-alkaline magmas? In the following, we will address this question by forward and inverse modelling of the slab flux.

Modelling the flux of REE and $Y$. Forward modelling the slab-to-arc flux provides one way to evaluate the impact of recycled trace elements on arc magmas. However, such modelling is complicated, because it involves many poorly known variables (e.g. Straub \& Zellmer 2012). The slab flux depends on slab composition, residual phases and partitioning behaviour of the individual elements, all of which vary strongly as the slab is processed at gradually increasing pressures and temperatures (e.g. Kessel et al. 2005; Klimm et al. 2008; Skora \& Blundy 2010). In order to limit the uncertainties inherent in forward modelling, we focused on the REE and Y. These elements have the advantage of behaving coherently in petrogenetic processes, span a wide range of incompatible to moderately incompatible elements, and are sensitive to residual garnet. While the inherent uncertainties do not allow one to obtain exact numbers, they allow for testing whether the REE and Y pattern of the arc magmas can be consistent with $>15 \%$ silicic slab component in the mantle source. Modelling parameters are compiled in Table 2; see also the Supplementary material.

The models calculate first the REE and Y of partial slab melts from the subducted sediment and basaltic igneous crust, respectively. (Note: We do not distinguish between a 'fluid-like' or 'melt-like' nature of the slab component, but prefer the term 'slab melt', because the mobilization of melt-mobile elements (LREE) is implied.) A composite of the two slab melts is then added to the 'background mantle', which is the subarc mantle prior to subduction modification. Finally, a partial melt of this metasomatized mantle is calculated, assuming source transformation from peridotite to a solid pyroxenite lithology prior to melting. Figure $8 \mathrm{com}-$ pares the modelled melts with target compositions of representative calc-alkaline basalts and andesites of the central MVB. Details of the models are as follows:

(1) Primitive mantle from McDonough \& Sun (1995), partially depleted by previous melting events, was chosen as background mantle. This choice is based on Straub et al. (2013), who identified the high-Nb basalts of the 'old Texcal Flow' (c. 17-19 ppm Nb), which erupts a few kilometres SE of Guespalapa, as the central MVB magma type least affected by slab metasomatism. These basalts are very similar to a c. 3-5\% partial melt of primitive mantle, but are too enriched in $\mathrm{TiO}_{2}$, LREE and HFSE to serve as source of the calc-alkaline magmas. However, residues of this mantle, which resemble depleted MORBtype mantle, have suitable abundances. While the subarc mantle could be inherently heterogeneous, we favour a melting link between enriched and depleted compositions because an overall compositional continuum exists between calc-alkaline and high- $\mathrm{Nb}$ magmas in the central MVB (LaGatta 2003; GomezTuena et al. 2007a). 
Table 2. Summary of trace element modelling

\begin{tabular}{|c|c|c|c|c|c|c|c|}
\hline $\begin{array}{l}\text { Sample_ID } \\
\text { Volcano } \\
\text { Eruptive unit }\end{array}$ & & $\begin{array}{l}\text { CH07-6 } \\
\text { Guespalapa } \\
\text { Manteca }\end{array}$ & $\begin{array}{l}\text { CH07-12 } \\
\text { Guespalapa } \\
\text { El Hoyo }\end{array}$ & $\begin{array}{l}\text { SPO60 } \\
\text { Cuatepel } \\
\text { Cone }\end{array}$ & $\begin{array}{l}\text { MCH06-9 } \\
\text { Guespalapa } \\
\text { El Caballito }\end{array}$ & $\begin{array}{l}\text { CH08-15 } \\
\text { Yecahuazac } \\
\text { Cone }\end{array}$ & $\begin{array}{l}\text { S15 } \\
\text { Tuxtepec } \\
\text { Cone }\end{array}$ \\
\hline $\begin{array}{l}\text { Background mantle (mantle } \\
\text { without subduction component) } \\
\text { from McDonough \& Sun (1995) }\end{array}$ & & $\begin{array}{l}\text { Residual mantle } \\
\text { after extraction of } \\
5 \% \text { melt }\end{array}$ & $\begin{array}{l}\text { Residual mantle } \\
\text { after extraction } \\
\text { of } 5 \% \text { melt }\end{array}$ & $\begin{array}{l}\text { Residual mantle } \\
\text { after extraction } \\
\text { of } 5 \% \text { melt }\end{array}$ & $\begin{array}{l}\text { Residual mantle } \\
\text { after extraction } \\
\text { of } 5 \% \text { melt }\end{array}$ & $\begin{array}{l}\text { Residual mantle } \\
\text { after extraction } \\
\text { of } 7 \% \text { melt }\end{array}$ & $\begin{array}{l}\text { Residual mantle } \\
\text { after extraction } \\
\text { of } 7 \% \text { melt }\end{array}$ \\
\hline \multirow{4}{*}{$\begin{array}{l}\text { Modal composition of } \\
\text { metasomatized mantle }\end{array}$} & GRT & - & - & - & $0.5 \%$ & $1 \%$ & $0.5 \%$ \\
\hline & OLIV & $2 \%$ & $10 \%$ & - & $20 \%$ & $14 \%$ & 16 \\
\hline & OPX & $90 \%$ & $80 \%$ & $90 \%$ & $67 \%$ & $80 \%$ & 83 \\
\hline & CPX & $8 \%$ & $10 \%$ & $10 \%$ & $12 \%$ & $5 \%$ & 1 \\
\hline $\begin{array}{l}\text { Ratio of sediment: igneous crust } \\
\text { in composite slab component }\end{array}$ & & 7: 93 & 10: 90 & 20: 80 & 25: 75 & 7: 93 & 10: 90 \\
\hline $\begin{array}{l}\text { Percentage of composite slab } \\
\text { component in metasomatized } \\
\text { mantle }\end{array}$ & & 30 & 25 & 25 & 15 & 20 & 30 \\
\hline $\begin{array}{l}\text { Extent of melting of } \\
\text { metasomatized mantle }(\%)\end{array}$ & & 7 & 7 & 5 & 3 & 2 & 3 \\
\hline Least squares fit* & & 0.68 & 0.60 & 0.77 & 0.49 & 0.66 & 0.84 \\
\hline \multirow{8}{*}{$\begin{array}{l}\text { Percentages of elements added } \\
\text { from slab }\end{array}$} & $\mathrm{La}$ & 91 & 90 & 93 & 90 & 93 & 97 \\
\hline & $\mathrm{Ce}$ & 83 & 82 & 86 & 79 & 86 & 94 \\
\hline & $\mathrm{Nd}$ & 72 & 68 & 73 & 61 & 71 & 86 \\
\hline & $\mathrm{Sm}$ & 61 & 56 & 60 & 45 & 53 & 76 \\
\hline & $\mathrm{Eu}$ & 52 & 46 & 48 & 34 & 41 & 67 \\
\hline & $\mathrm{Y}$ & 13 & 10 & 11 & 6 & 9 & 24 \\
\hline & $\mathrm{Yb}$ & 5 & 4 & 4 & 2 & 4 & 11 \\
\hline & $\mathrm{Lu}$ & 4 & 3 & 3 & 2 & 3 & 11 \\
\hline
\end{tabular}

*The least squares fit assesses the fit between model and measured composition. For each element, the values of $x$ was calculated as follows: $x=(($ measured values - modelled value $) / 10 \%$ of measured values) ${ }^{2}$. If the average of $x$ for all elements was $<1$, the fit was considered satisfactory.

For other reservoir compositions and partition coefficients, see Supplementary material. 

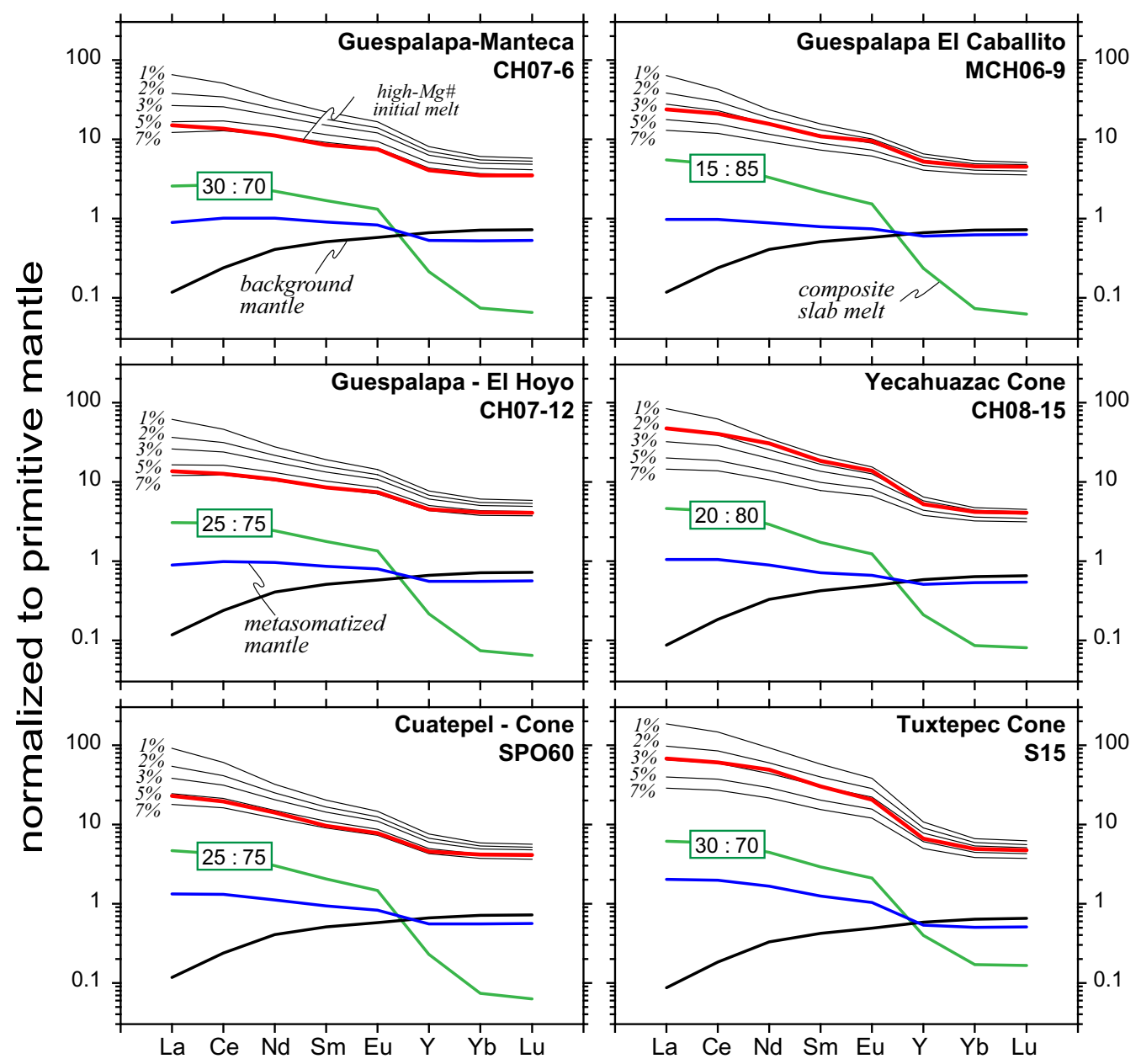

Fig. 8. Model calculations of REE and Y in selected low-La and high-La calk-alkaline andesites. Modelling and input parameters are given in Table 2 and in the Supplementary material. Data normalized to primitive mantle of McDonough \& Sun (1995). Thick red line, composition of high-Mg\# arc magma; thin black lines, forward models for 1, 2, 3, 5 and 7\% extents of melting; thick green line, composite slab component of melts from subducted sediment melt and igneous crust, with the mixing ratio displayed in box; thick black line, background mantle; thick blue line, metasomatized mantle, which means background mantle and slab component. For discussion see text.

(2) We use the average terrigenous trench sediment from LaGatta (2003). For subducted basaltic igneous crust, we use the average recycled MORB from East Pacific Rise from Donnelly et al. (2004) (note that the N-type MORB from Sun \& McDonough (1989) also produces acceptable results for most samples). No loss of REE and Y beneath the forearc was considered, which is consistent with the fluid-immobile nature of these elements. Partial slab melts were calculated with the batch melting equation after Langmuir et al. (1992) and the experimentally determined bulk partition coefficients $(D)$ for elements La, Ce, Nd, Sm, Eu, Y, Yb and Lu from Skora \& Blundy (2010) (sediment) and Klimm et al. (2008) (basaltic igneous crust), which were obtained in the same laboratory by similar techniques. All models use a $5 \%$ partial melt of either sediment or igneous crust.

(3) A composite of the partial melts from sediment and basaltic crust was calculated and added to the subarc mantle. The mixing ratio of the two slab components in the composite melt is arbitrary, and was adjusted to acquire the best results. Finally, we assumed an amount of c. $15-30 \%$ of composite slab 
melt in the mantle source, which corresponds to the percentages required by the pyroxenite model.

(4) Melting of the metasomatized mantle was done by a batch melting model after Langmuir et al. (1992) with mineral-melt partition coefficients $\left(K_{\mathrm{d}}\right)$ from Donnelly et al. (2004). Olivine, orthopyroxene, clinopyroxene and sometimes traces of garnet were residual phases. The proportion of olivine and orthopyroxene varied in agreement with the reaction model, but had no perceptible effect on the REE and Y abundances in the melt, because of the very similar $K_{\mathrm{d}}$ values of olivine and orthopyroxene.

(5) The modelled melt compositions were compared with representative calc-alkaline basalts and andesites with high- ${ }^{3} \mathrm{He} /{ }^{4} \mathrm{He}$ olivines. The high values of $\mathrm{Mg} \#>65.8-72.8$ of these magmas suggest only minimum loss of mafic silicates from the initial melts, and hence they should closely resemble the initial mantle melts. Initial melt compositions were calculated by adding equilibrium olivine in $1 \%$ steps until the maximum $\mathrm{Mg} \#=72.8$ observed (corresponds to olivine with $\mathrm{Fo}_{89.8}$ ). The REE and $\mathrm{Y}$ were corrected accordingly, assuming perfect incompatibility in olivine. The agreement of the modelled melts with fractionation-corrected 'target magmas' was tested by a least squares fit calculated as follows for each element: $x=$ ((measured values - modelled value) $/ 10 \%$ of measured values). An average of $\leq 1$ for all values of $x$ - which means that results are within $10 \%$ of observed data - was considered satisfactory. The principal results of the forward models are shown in Figure 8 and can be summarized as follows:

(a) The strong garnet signature of the slab melts is not transmitted to arc magmas, even if the mantle source contained as much as $15-40 \%$ of the composite melt. This is due to the fact that the high values of $D$ of the slab (see Supplementary material) efficiently retain the HREE in the slab. Consequently, the HREE ( $c$. Ho to $\mathrm{Lu}$ ) of the background mantle are an order of magnitude higher than those of the slab melts, and the mantle HREE dominate the HREE budget of the partial melts. This results in the flat HREE observed. On the other hand, as suggested by many studies (e.g. Elliott et al. 1997, and later studies), the slab flux significantly increases the LREE in the subarc mantle, up to a point where the slab flux controls the LREE in the arc magmas, especially if the background mantle was depleted by previous melting.

(b) There is no problem of reproducing the REE and Y patterns of the low-La calcalkaline basalts and andesites. In these models, values of the slab bulk $D$ were always the same, and the proportions of sediment melt in the slab composite melt $(7-25 \%)$ and of composite slab component in mantle (15-30\%) were similar. Only one model (for sample MCH06-9) required residual garnet $(0.5 \%)$ in the mantle source. The extent of mantle melting c. $3-7 \%$ is fairly low, but is probably underestimated because the model results depend here on the choice of bulk $D$ of slab. The high values of $D$ for the igneous crust (obtained at $2.5 \mathrm{GPa}$, Klimm et al. 2008) require lower extents of melting, but the lower $D$ at the higher pressures of c. $3 \mathrm{GPa}$ more appropriate for arc front depth allows for extents of mantle melting $>5 \%$.

(c) The steeper REE patterns of the high-La basalts from Tuxtepec and Yecahuazac Cone are more difficult to model. Notably, the problem is not so much matching their steeper HREE, but their fairly flat LREE. A different choice of bulk $D$ for sediment melting from Skora \& Blundy (2010) produces acceptable results, but requires low extents of mantle melting $(2-3 \%)$ and a comparatively large proportion of sediment in the slab composite melt $(10-25 \%)$, as well as a large proportion of composite slab melt $(20-30 \%)$ in mantle source. Again, these latter three variables all depend on the bulk $D$ values of the LREE which are highly variable and uncertain across the relevant $P-T$ range. Unfortunately, only measured bulk $D$ can be used (Kessel et al. 2005; Klimm et al. 2008; Skora \& Blundy 2010), as partitioning data for clinopyroxene, a major residual phase in slab, are not available. Calculated bulk $D$, from the sum of the individual values of $K_{\mathrm{d}}$, that includes the dependency on slab temperature, pressure and proportion of residual phases, may provide better results. Thus, despite the successful fit with still reasonable parameters, the numbers derived by the model must be considered as approximations, and not as a constraint. 
In summary, the REE signature of the calcalkaline MVB magmas can be reconciled with the large amounts of slab component in source required by the pyroxenite model. This result is a consequence of the high $D$ values $>1$ for the two slab components and, in particular, of the high $D$ values of the HREE which reach 100 and more (see Supplementary material). At such high $D$ values, the composite slab component is effectively devoid of the HREE so that the HREE of the background mantle control the HREE in melts from metasomatized mantle. Such results cannot be achieved with lower slab $D$ values $<1$ (e.g. Stolper \& Newman 1994; Gomez-Tuena et al. 2007a). For example, the calculation of GomezTuena et al. (2007a) used slab $D$ values that are lower by an order of magnitude. At such low $D$ values, the mantle wedge can absorb only up to $10 \%$ slab component without transmitting a garnet signature to a partial melts, and additional 'chromatographic' exchange between the advancing slab component and the surrounding mantle 'column' was required to obtain a more mantle-like composition (e.g. Stolper \& Newman 1994). On the other hand, the forward calculations predict that $>50-$ 98\% of the LREE in the central MVB magmas were slab-derived (Table 2). Thus, despite the high slab $D$ values of Skora et al. (2010) and Klimm et al. (2008), the slab flux still strongly affects the composition of the subarc mantle.

The magnitude of the slab flux: the inverse approach. LREE are considered as 'melt-mobile', and hence it seems likely that other melt-mobile elements, such as the MREE and HFSE, were also added from slab in significant amounts (Elliott et al. 1997; Woodhead et al. 1998, 2001; Plank 2005; Gomez-Tuena et al. 2007a, 2011). Forward modelling, however, is too uncertain for deriving robust information on the mobility of the MREE and HFSE. Therefore, we test this hypothesis with the inverse approach of Pearce et al. (1995). This method assumes that the ratios of incompatible trace elements in arc magmas resemble those of the mantle source. Partial melts from 'background mantle' (which is the mantle without slab components) thus have 'smooth' patterns of (normalized) incompatible elements, whereas the mantle infiltrated by slab components produces arc magmas with strong relative enrichment of slab-derived elements. The different abundance of a given element between melts from the background and the metasomatized mantle, respectively, then provides a direct measure of the percentage of the element contributed from slab.

An important reference in this approach is the trace element composition of the melt from the unmetasomatized background mantle. Such melts commonly do not erupt in arcs, but their trace element patterns can be reconstructed from the erupted arc magmas on the basis of few elements that are principally mantle-derived in a given set of arc magmas. Pearce et al. (1995) used $\mathrm{Nb}$ and $\mathrm{Yb}$ to this purpose, assuming these elements to be entirely mantle-derived. While this works well in some arcs, a principal mantle origin (or 'conservative' behaviour) of $\mathrm{Nb}$ is doubtful in the central MVB. One immediate problem is the huge range in $\mathrm{Nb}$ of a factor of 10 (from 3.6 to $33.5 \mathrm{ppm} \mathrm{Nb}$ ) in high-Mg\# $>60$, high $-{ }^{3} \mathrm{He} /{ }^{4} \mathrm{He}$ magmas, that erupt within a few kilometres and a few thousand years from each other (Fig. 9) (Straub et al. 2013, and this study). This range of $\mathrm{Nb}$ cannot result from different extents of melting, fractional crystallization or crustal contamination, but must reflect mantle source heterogeneity. Moreover, slab signatures emerge in the magmas with the highest and lowest $\mathrm{Nb}$, while the magmas least influenced by slab additions (the 'old Texcal Flow basalts') have intermediate $\mathrm{Nb}$ c. 17-19 ppm (Fig. 9). Notably, magmas with higher $\mathrm{Nb}>20 \mathrm{ppm}$ also have higher ratios of $\mathrm{Nb}-\mathrm{Ta}>17$ that have been related to the addition of high $\mathrm{Nb}-\mathrm{Ta}$ components from slab (e.g. Stolz et al. 1996; Gomez-Tuena et al. 2011; Koenig \& Schuth 2011).

On the other hand, $\mathrm{TiO}_{2}$ abundances and variations are consistent with a principal mantle origin. The old Texcal Flow basalts have the highest $\mathrm{TiO}_{2}=1.96 \mathrm{wt} \%$ and high $\mathrm{TiO}_{2}-\mathrm{Lu}=4.6$ in central Mexico (Fig. 9, Straub et al. 2013), while all other HNABs and calc-alkaline magmas have lower $\mathrm{TiO}_{2}$ of $<2-0.8 \mathrm{wt} \%$, and $\mathrm{TiO}_{2}-\mathrm{Lu}<4$.62.5. These ranges are similar to those of MORB, which provides a reference for the variability of melts derived from an unmetasomatized upper mantle (Pearce et al. 1995; Pearce \& Peate 1995). Therefore, we take $\mathrm{TiO}_{2}-\mathrm{Lu}$ to approximate the MVB background mantle. The results are shown in Figures 10 and 11 , where $\mathrm{TiO}_{2}-\mathrm{Lu}$ is plotted against the Lu-normalized element investigated for slab addition. If the investigated element - which is the numerator of the ratio on the $y$-axis - is mantle-controlled like $\mathrm{TiO}_{2}$ and $\mathrm{Lu}$, the MVB data will overlap with mantle magmas. When the third element is added from slab, it shows a positive deviation from the range of the mantle melts that scales to the percentage of the slab-derived element in the arc magmas.

Calculation of the background mantle. We assume the background mantle to be primitive mantle based on the nearby erupting old Texcal Flow basalts which resemble a $3.5 \%$ melt from primitive mantle with minimal slab additions (Straub et al. 2013). Assuming a pyroxenite source 


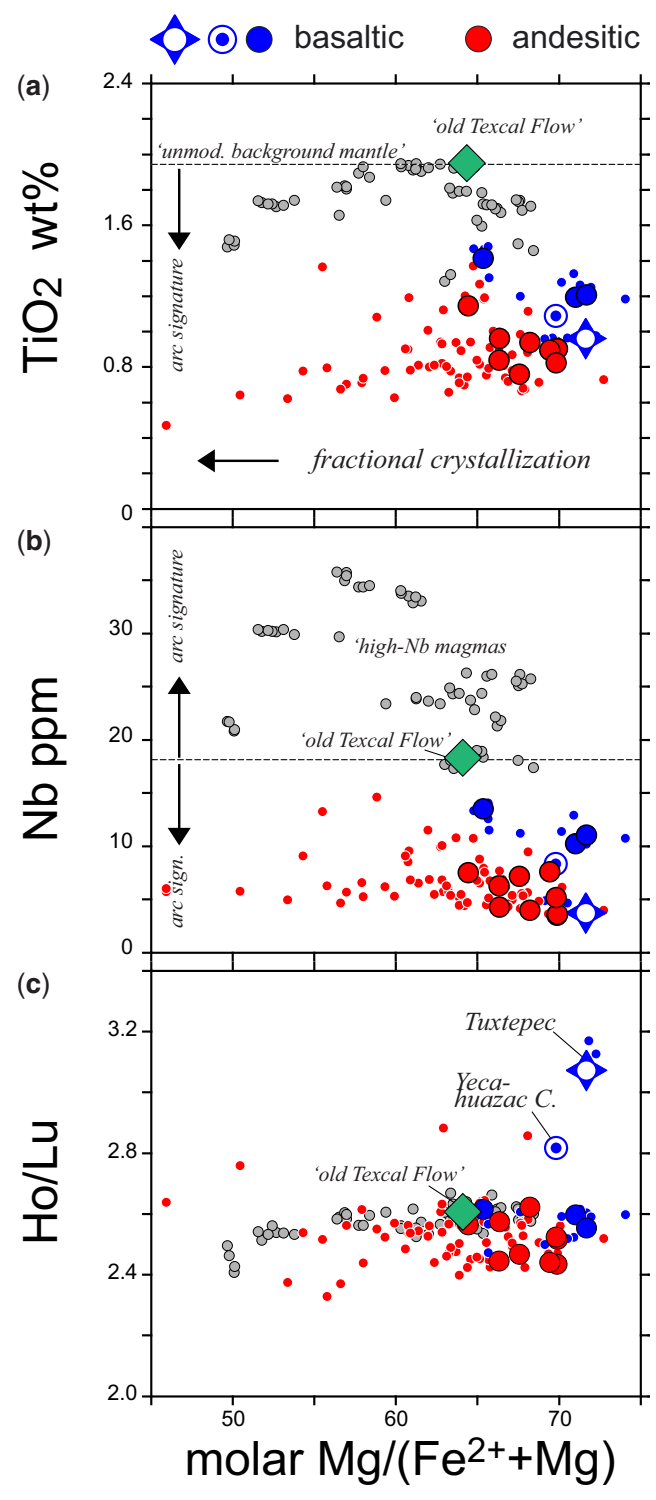

Fig. 9. (a-c) $\mathrm{Nb}(\mathrm{ppm}), \mathrm{TiO}_{2}(\mathrm{wt} \%)$ and $\mathrm{Ho} / \mathrm{Lu}$ v. $\mathrm{Mg \#}$ of central MVB calc-alkaline basalts and andesites. High- $\mathrm{Nb}$ arc magmas (grey circles) of the central MVB after Straub et al. (2013), LaGatta (2003) and this study. Old Texcal Flow magma is a high-Nb arc basalt from Straub et al. (2013) that approximates best a melt from the central MVB mantle prior to subduction processing. Stippled horizontal lines abundance levels of melts from unmodified background mantle prior to subduction modification (which is similar to primitive mantle). In mantle-controlled elements, depletion by melting can only lower the abundance in later melts (e.g. $\mathrm{TiO}_{2}$ ). The existence of the high- $\mathrm{Nb}$ magmas thus indicates $\mathrm{Nb}$ addition from slab, which is consistent with the 'arc signatures' of the magmas (e.g. Nb/La) decreasing above and below the 'background mantle line'. lithology (orthopyroxene-clinopyroxene-garnet $=$ 85:15:0.3) and the $K_{\mathrm{d}}$ values of Donnelly et al. (2004), we then calculated a range of melts from this primitive mantle with different extents of melting, ranging from 3.5 to $5,10,20$ and $50 \%$. Figure 10 shows that the lowest $\mathrm{TiO}_{2} / \mathrm{Lu}$ observed is not reproduced by even an extent of melting of $50 \%$, which is unrealistically high. However, the low $\mathrm{TiO}_{2} / \mathrm{Lu}$ can be reproduced assuming re-melting of a residual mantle after several per cent melt extraction. Repetitive mantle melting, triggered by new hydrous slab additions, is a realistic model for the central MVB that is characterized by closely spaced, successive volcanic eruptions (e.g. Siebe et al. $2004 b$, 2005). The exact amount of the previously extracted melt and the number of melting events is not known, but it serves to assume 5\% melt extraction in order to illustrate the resulting chemical variations. Melting curves for the original (primitive) mantle (solid lines), and for the depleted residual mantle (stippled) are shown in Figures 10 and 11 together with arc magmas with $\mathrm{Mg \#}>60$.

Highly incompatible elements. Figure 10 illustrates the behaviour of highly incompatible elements (those to the left of $\mathrm{Sr}$ in multi-element diagrams) by the examples of $\mathrm{Th}, \mathrm{Nb}$ and $\mathrm{K}_{2} \mathrm{O}$. Note that data are filtered for $\mathrm{Mg \#}>60$, which minimized the influence of crustal contamination. The interpretation of the Lu-normalized ratios of these elements requires some caution for two reasons. First, these ratios increase at an extent of melting $<10 \%$ during melting, but still to a far lesser extent than the increase caused by slab additions. Second, the Lu-normalized ratios differ significantly in melts from enriched and depleted mantle sources, respectively (Fig. 10). However, despite these limitations, it is obvious that these elements must be slab-controlled. Even relative to an enriched, primitive mantle - which illustrates the minimum slab addition - the slab-derived percentages in the magmas exceed $>50 \%$ (except for $\mathrm{Nb}$ and $\mathrm{Ta}$ ). If the mantle is depleted by previous melting, the slab-derived percentages commonly exceed $>90 \%$ and approximate $100 \%$ in many cases. The case of $\mathrm{Nb}$ (and Ta) is different, as a primitive mantle cannot produce the low $\mathrm{Nb} / \mathrm{Lu}$ of some of the calc-alkaline magmas (Fig. 10b). However, after the loss of only a few per cent of melts, the residual mantle is practically devoid of $\mathrm{Nb}$, and very sensitive to leverage by the slab flux. Even a slab component with low Nb relative to the other highly incompatible elements may then add significant $\mathrm{Nb}$ to the mantle. While percentages of slab-derived $\mathrm{Nb}$ in arc magmas may not be as high as those of the fluid-mobile elements, slab-derived $\mathrm{Nb}$ may still account for $>50 \%$ of the low- $\mathrm{Nb}$ arc magmas with only $3-4 \mathrm{ppm} \mathrm{Nb}$ (Fig. 10b). If $\mathrm{Nb}$ 
was mobilized to the same extent as the other highly incompatible elements, the slab flux of $\mathrm{Nb}$ must clearly control $\mathrm{Nb}$ abundances in arc magmas. In summary, while exact percentages of the slab-derived highly compatible elements are difficult to assess, the inverse approach suggests that slab flux of these elements dominates the arc outflux, and especially so if the mantle has been depleted by previous melting.

Moderately incompatible elements. For moderately incompatible elements (to the right of $\mathrm{Sr}$ in

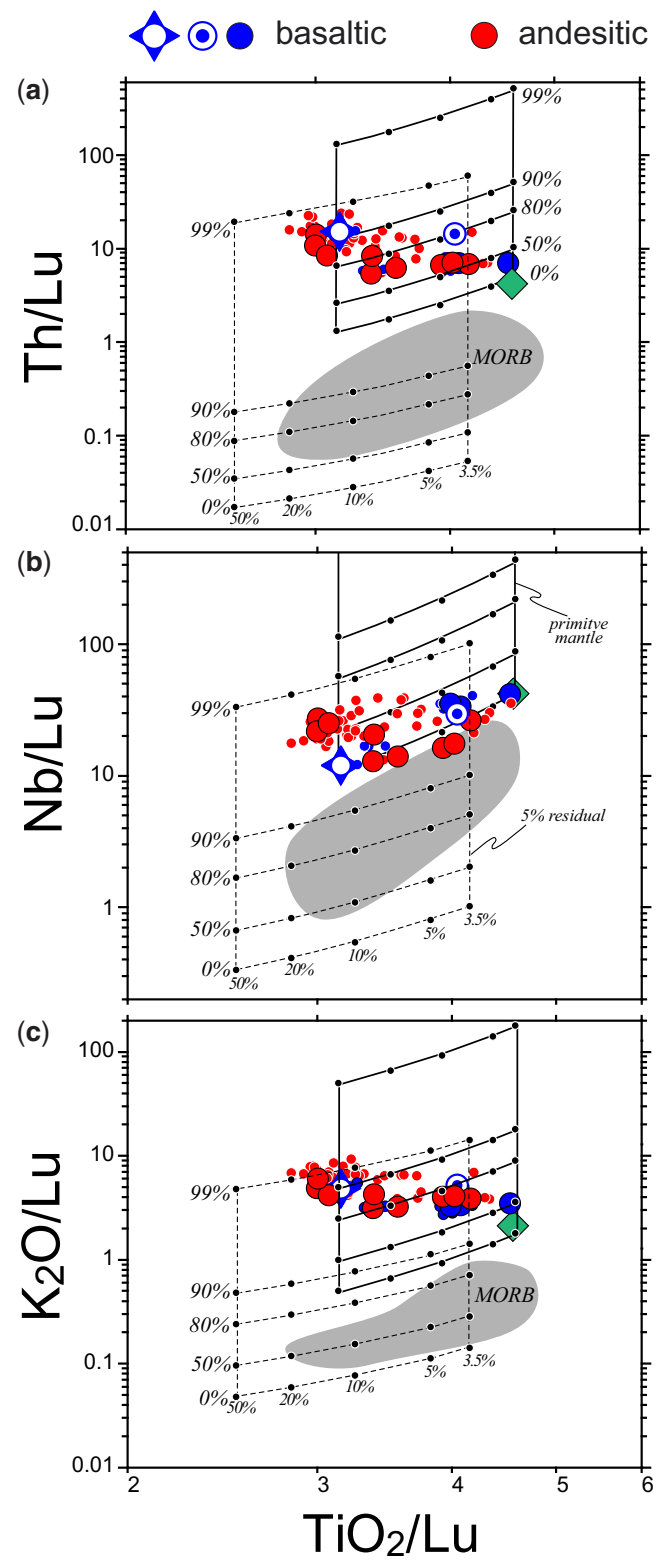

multi-element diagrams), which include the MREE and HREE, previous melt extraction is far less efficient in modifying the Lu-normalized ratios because their values of $D$ are less different from $D_{\mathrm{TiO} 2}$ during mantle melting (Fig. 11). Thus, the assessment against $\mathrm{TiO}_{2} / \mathrm{Lu}$ provides fairly robust estimates of the slab-derived percentages of the elements in the arc magmas.

The inversion indicates significant slab additions for the middle REE down to Gd, Tb and Dy. In general, between $c$. 20 and $90 \%$ of these elements may be slab-derived in the low-La calc-alkaline magmas, whereby the slab-derived percentage decreases with decreasing incompatibility. The high-La basalts have high slab contributions of these elements, which suggest the presence of rare, unusually enriched slab components. Notably, the inversion indicates that as much as $50 \%$ of the Gd (placed just to the right of $\mathrm{TiO}_{2}$ in multielement diagrams) may be slab-derived low-La calc-alkaline magmas (and up to $70 \%$ in the highLa basalts). Thus, recycling of Eu (just to the right of $\mathrm{TiO}_{2}$ in multi-element diagram) and $\mathrm{Gd}$ from slab, combined with $\mathrm{TiO}_{2}$ retention in the slab, would cause the pronounced negative $\mathrm{TiO}_{2}$ anomaly relative to Eu and Gd (Fig. 4). In other words, while the $\mathrm{Eu}$ and $\mathrm{Gd}$ abundances in arc magmas reflect both mantle and slab contributions, $\mathrm{TiO}_{2}$ varies only by depletion through melting. Indeed, no alternative process can account for the relative $\mathrm{TiO}_{2}$ depletion in the high-Mg\# magmas. Fractional crystallization, different extents of partial melting and crustal contamination are all precluded by the significant range of $\mathrm{TiO}_{2} / \mathrm{Gd}$ at comparable high Mg\# values.

The comparative robustness of the Lu-normalized ratios of the moderately incompatible elements towards the background mantle allows for correlating their slab-derived percentages with melt

Fig. 10. (a-c) Lu-normalized ratios of $\mathrm{Th}, \mathrm{Nb}$ and $\mathrm{K}_{2} \mathrm{O}$ v. $\mathrm{TiO}_{2} / \mathrm{Lu}$ for central MVB calc-alkaline basalts and andesites. All samples have Mg\# $>60$. Field of MORB with $<10$ ppm Nb after Niu \& Batiza (1997). Raster with solid lines $x$-axis indicates variations of $3.5,5,10,20$ and 50\% melts (sub-horizontal lines) from primitive mantle after McDonough \& Sun (1995); partition coefficient from Donnelly et al. (2004). Residual source: orthopyroxene-clinopyroxene-garnet, 85:15:0.3. Vertical lines along $y$-axis indicate percentage of element derived from subducted slab. Raster with stippled lines - as before, only assuming residual primitive mantle after $5 \%$ melt extraction as source with orthopyroxene-clinopyroxene-garnet, 95:5:0.3. Old Texcal Flow magma (large green diamond) is a high-Nb arc basalt of the Sierra Chichinautzin that is only minimally affected by slab additions and best represents a melt from background mantle prior to subduction processing (Straub et al. 2013). 


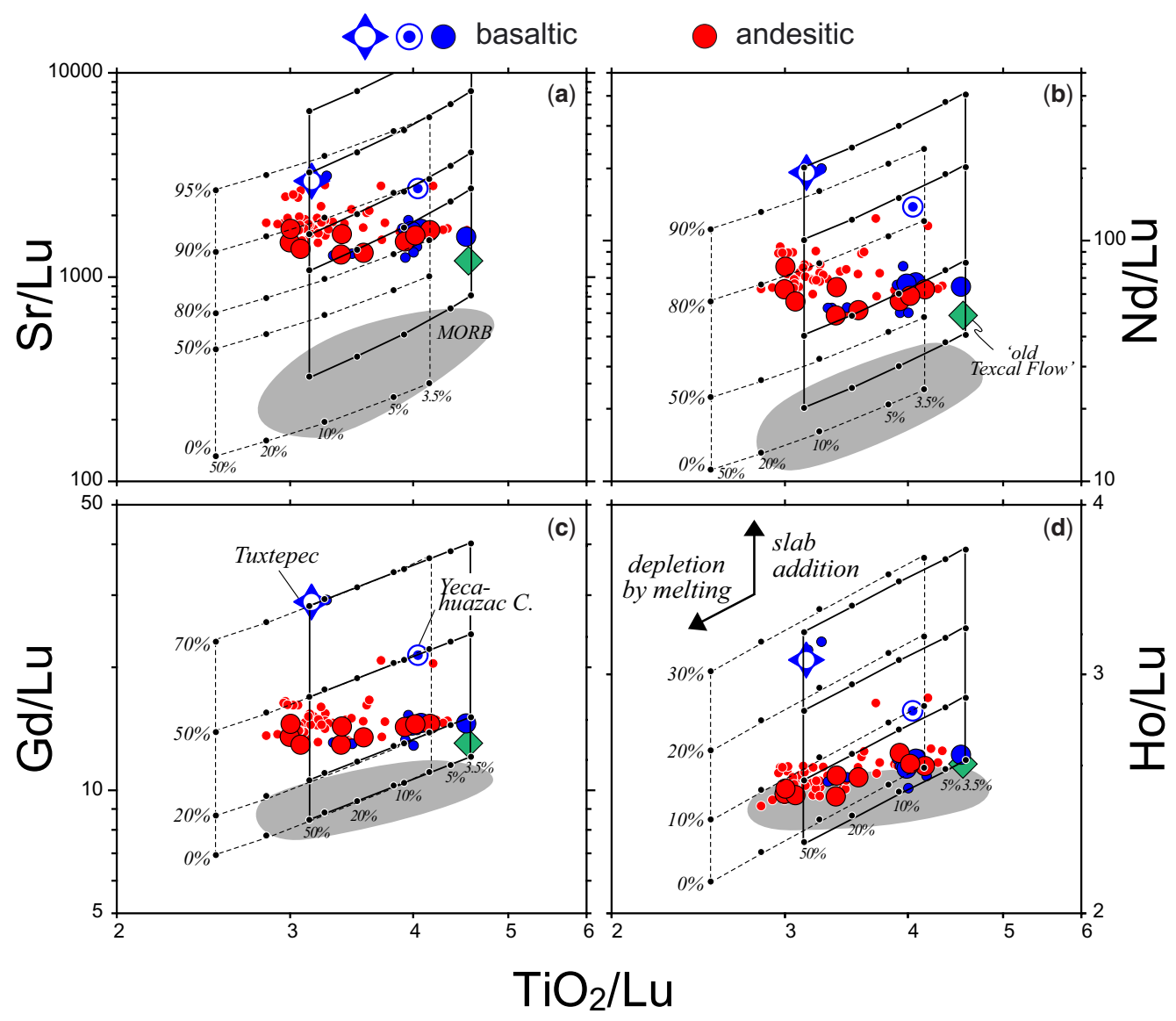

Fig. 11. (a-d) Lu-normalized ratios of $\mathrm{Nd}, \mathrm{Sr}, \mathrm{Gd}$ and $\mathrm{Ho}$ v. $\mathrm{TiO}_{2} / \mathrm{Lu}$ for central $\mathrm{MVB}$ calc-alkaline basalts and andesites. All other variables as in Figure 10.

$\mathrm{SiO}_{2}$. In the low-La calc-alkaline magmas, the slabderived percentage increases with melt $\mathrm{SiO}_{2}$ as predicted by forward models (Fig. 12). As expected, the slope of the increase v. melt $\mathrm{SiO}_{2}$ becomes flatter, as the percentage of the slab-derived element decreases, and the subarc mantle increasingly buffers the lesser slab flux of the least incompatible elements. Notably, in agreement with the forward models, only the HREE to the right of Ho (Er and $\mathrm{Yb}, \mathrm{Lu}$ by definition) lack correlation with melt $\mathrm{SiO}_{2}$, which is consistent with their principal origin from background mantle and their efficient retention in the subducted slab by residual garnet.

In the high-La basalts from Tuxtepec and Yecahuazac Cone the slab flux increases independently from melt $\mathrm{SiO}_{2}$ (Fig. 12). However, these excursions are accountable in the context of the pyroxenite model, when considering that the slab flux must not be homogenous. Such extreme enrichments may simply reflect rare slab components that are extremely enriched in REE and some other incompatible elements (e.g. $\mathrm{Sr}, \mathrm{P}_{2} \mathrm{O}_{5}$ ), but depleted in the $\mathrm{Nb}$ and $\mathrm{Ta}$. If the mantle was already depleted by melting, the full signature of this slab component must then show against the background, including the garnet signature. Moreover, in this case the high element abundances do not depend on multiple infiltrations of silicic slab components, and the addition of one such extremely enriched component may suffice to create silicadeficient pyroxenite that then produces enriched basaltic melts. Obviously, the rarity of the high-La basalts shows that these conditions of formation are not commonly met, in contrast to the more ubiquitous flux of slab components that produce the low-La calc-alkaline series.

In summary, the inverse approach agrees with the results of the forward models in that most incompatible elements are either completely or strongly controlled by the slab flux. Only a few elements, 


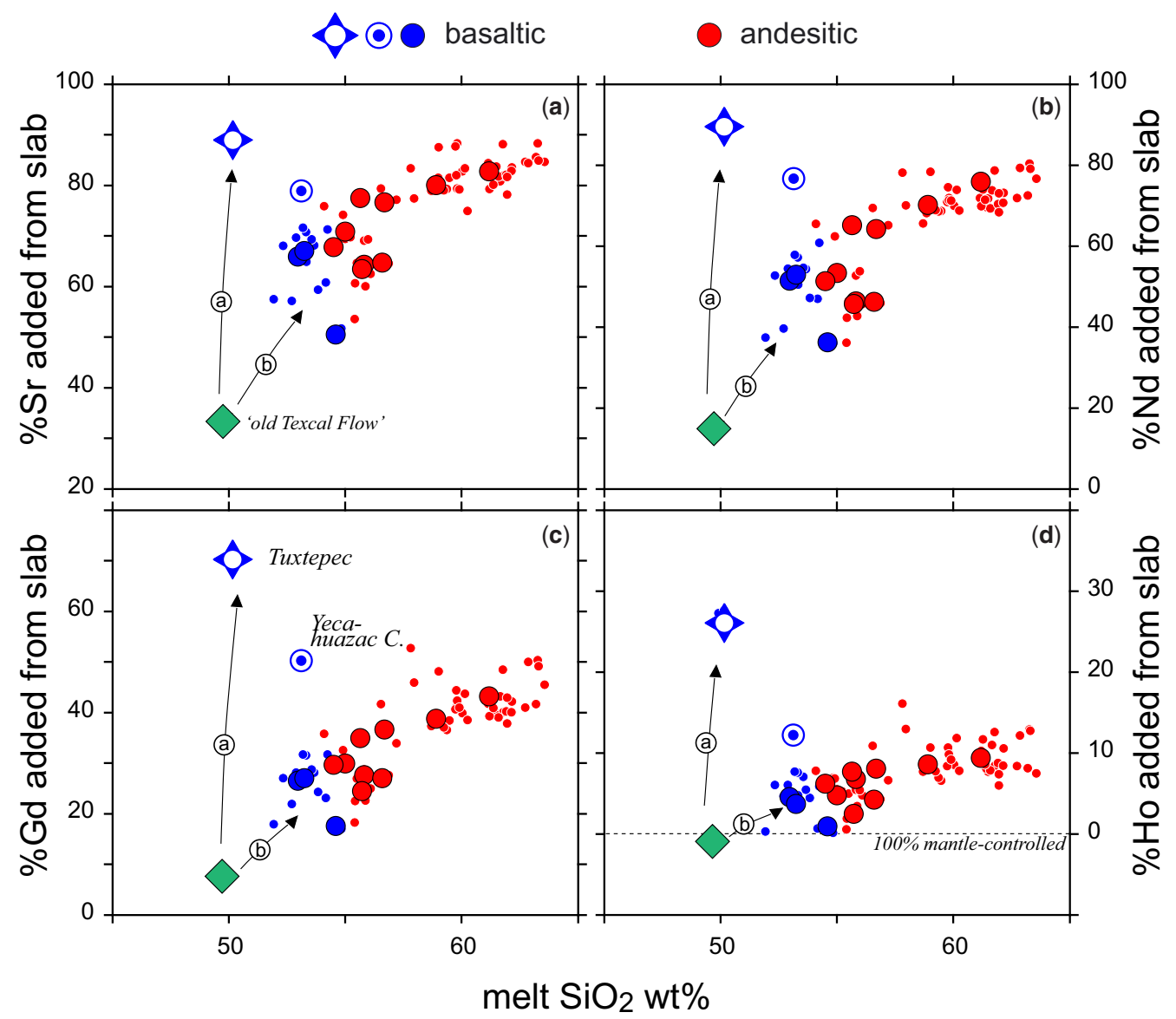

Fig. 12. (a-d) Percentage of moderately incompatible slab-derived elements $\mathrm{Sr}, \mathrm{Nd}, \mathrm{Gd}$ and Ho in arc magmas v. melt $\mathrm{SiO}_{2}$ for samples with $\mathrm{Mg} \#>60$. Old Texcal Flow magma (large green diamond) is a high-Nb arc basalt from Straub et al. (2013) that is only minimally affected by slab additions and best represents a melt from the central MVB background mantle prior to subduction processing. Trend labelled ' $a$ ' indicates addition of highly enriched slab melt to silica-deficient 'reaction pyroxenite' producing basaltic melts. Trend labelled 'b' indicates addition of multiple silicic slab components that create silica-excess pyroxenite. Note transitional character of Yecahuazac Cone basalt.

such as $\mathrm{TiO}_{2}$ and the HREE Er, $\mathrm{Yb}$ and Lu principally derive from mantle, which accounts for the flat HREE patterns and the negative $\mathrm{TiO}_{2}$ anomaly in the arc magmas. Additional variability can be attributed to rare, extremely enriched slab components that infiltrate a subarc mantle that has already been depleted by melting.

Sr and Nd isotope mixing trends. Lastly, we test these results in $\mathrm{Sr}-\mathrm{Nd}$ isotope space. It has been suggested that the moderate deviations of ${ }^{87} \mathrm{Sr} /$ ${ }^{86} \mathrm{Sr}$ and ${ }^{143} \mathrm{Nd} /{ }^{144} \mathrm{Nd}$ from MORB-type mantle magmas were caused by crustal contamination (e.g. Martinez-Serrano et al. 2004; Siebe et al. 2004a, 2005; Schaaf \& Carrasco-Núñez 2010). Clearly, on the basis of the ${ }^{87} \mathrm{Sr} /{ }^{86} \mathrm{Sr}$ and
${ }^{143} \mathrm{Nd} /{ }^{144} \mathrm{Nd}$ data alone, it is not possible to distinguish conclusively between the models. However, the entirety of the data available for this sample set allows for considering a possible slab control, and justifies a detailed discussion.

Both forward and inverse models suggest that most of $\mathrm{Sr}(c .50-80 \%)$ and $\mathrm{Nd}(c .40-80 \%)$ in the low-La calc-alkaline magmas were slab-derived, with the slab addition being even higher in the high-La magmas (Figs $11 \& 12$ ). Consequently, the moderate deviations of ${ }^{87} \mathrm{Sr} /{ }^{86} \mathrm{Sr}$ and ${ }^{143} \mathrm{Nd} /{ }^{144} \mathrm{Nd}$ from upper mantle should largely be controlled by the slab flux (Fig. 13). However, if subducted sediment was the only source of slab-derived $\mathrm{Sr}$ and $\mathrm{Nd}$ in the arc, the ${ }^{87} \mathrm{Sr} /{ }^{86} \mathrm{Sr}$ and ${ }^{143} \mathrm{Nd} /{ }^{144} \mathrm{Nd}$ of arc should be much closer to the trench sediment 


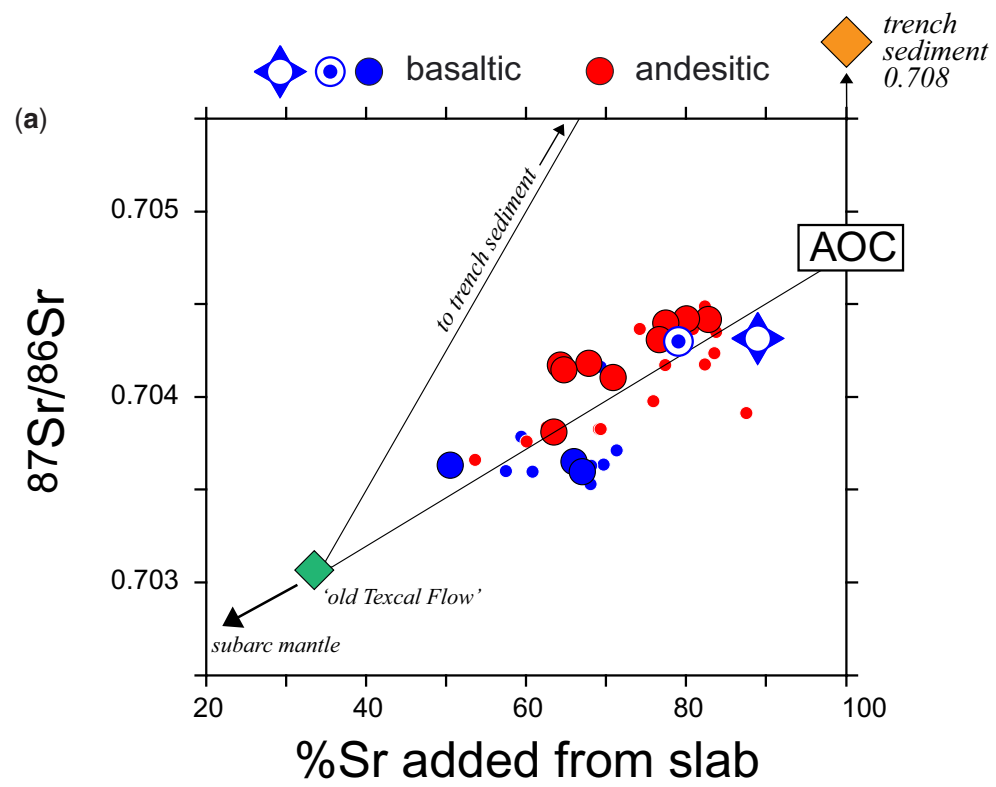

(b)

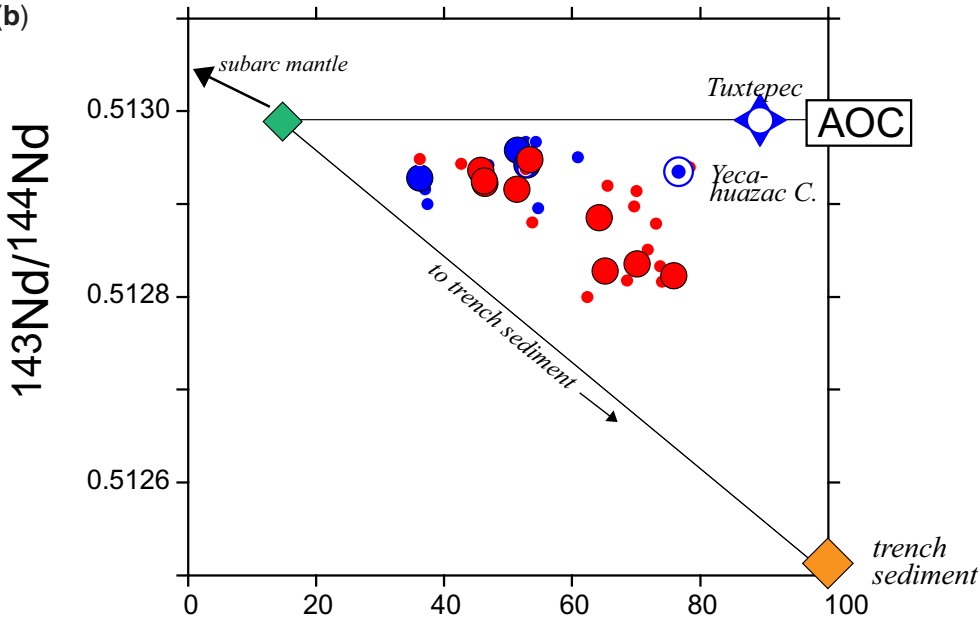

$\%$ Nd added from slab

Fig. 13. (a, b) Percentage of slab-derived elements $\mathrm{Sr}$ and $\mathrm{Nd}$ in the $\operatorname{arc}$ magmas v. ${ }^{87} \mathrm{Sr} /{ }^{86} \mathrm{Sr}$ and. ${ }^{143} \mathrm{Nd} /{ }^{144} \mathrm{Nd}$, respectively. All samples have $\mathrm{Mg \#}>60$. AOC, altered oceanic crust. Trench sediment after Gomez-Tuena et al. (2003). Old Texcal Flow magma (large green diamond) is a high-Nb arc basalt from Straub et al. (2013) that is only minimally affected by slab additions and best represents a melt from the central MVB background mantle prior to subduction processing.

than observed. On the other hand, the $\mathrm{Sr}$ and $\mathrm{Nd}$ flux from sediment can be buffered by the concomitant $\mathrm{Sr}$ and $\mathrm{Nd}$ flux from the far more voluminous subducted igneous crust, which results in fairly unradiogenic ${ }^{87} \mathrm{Sr} /{ }^{86} \mathrm{Sr}$ and radiogenic ${ }^{143} \mathrm{Nd} /{ }^{144} \mathrm{Nd}$, despite a high slab flux (e.g. Straub \& Zellmer 2012). Figure 13 correlates the percentage of slab-derived $\mathrm{Sr}$ and $\mathrm{Nd}$ with their respective isotope ratios. In both diagrams, the arc data do not plot on a mixing line between mantle and trench sediment, but show deviations that agree with the addition of $\mathrm{Nd}$ and $\mathrm{Sr}$ from the subducted altered igneous crust (referred to as 'AOC' (altered oceanic crust) hereafter). In $\% \mathrm{Sr} v .{ }^{87} \mathrm{Sr} /{ }^{86} \mathrm{Sr}$ space, the 
Sr-rich slab component has only moderately elevated ${ }^{87} \mathrm{Sr} /{ }^{86} \mathrm{Sr}$, c. 0.7048 . As suggested previously for the MVB (Gomez-Tuena et al. 2007a), this points to the AOC $\left({ }^{87} \mathrm{Sr} /{ }^{86} \mathrm{Sr}\right.$ up to $c$. 0.705 , e.g. Staudigel et al. 1995) as a principal source of the excess arc $\mathrm{Sr}$, and not the subducted trench sediment. Because ${ }^{143} \mathrm{Nd} /{ }^{144} \mathrm{Nd}$ remains unchanged during the alteration of the oceanic crust, $\mathrm{Nd}$ flux of the trench sediment must be responsible for the lower ${ }^{143} \mathrm{Nd} /{ }^{144} \mathrm{Nd}$ of the arc magmas. However, there is no clear trend to the trench sediment either. All arc data - and especially the high-La basalts - require radiogenic $\mathrm{Nd}$ from slab which must derive from the AOC.

Importantly, if $\mathrm{Sr}$ and $\mathrm{Nd}$ were added from igneous subducted crust as well, mixing relationships in the $\mathrm{Sr}-\mathrm{Nd}$ isotope space are complicated, because two of the source components (mantle and igneous crust) overlap in $\mathrm{Sr}$ and $\mathrm{Nd}$ isotope space. Quantification is further complicated by the fact that the arc data require the slab components to have different ratios of $\mathrm{Sr} / \mathrm{Nd}$. For example, the low-La calc-alkaline magmas have higher $\mathrm{Sr} /$ $\mathrm{Nd}=22-27$ than the high-La basalts (low $\mathrm{Sr}$ / $\mathrm{Nd}=15-20)$. These ratios are all higher than the $\mathrm{Sr} / \mathrm{Nd}$ of subarc mantle $(\mathrm{Sr} / \mathrm{Nd}=16)$, subducted igneous crust $(\mathrm{Sr} / \mathrm{Nd}=12.3)$ and trench sediment $(\mathrm{Sr} / \mathrm{Nd}=7)$, and thus agree with the idea of preferential $\mathrm{Sr}$ release from slab relative to the less mobile $\mathrm{Nd}$. However, it is also clear that the extreme enrichment of the high-La basalts requires a slab component that is enriched both in $\mathrm{Sr}$ and $\mathrm{Nd}$, but only minimally fractionated.

Because there are so many variables, there are only possible, not definite, solutions in the twodimensional ${ }^{87} \mathrm{Sr} /{ }^{86} \mathrm{Sr}$ v. ${ }^{143} \mathrm{Nd} /{ }^{144} \mathrm{Nd}$ space. Figure 14 presents two possible solutions for low-La calc-alkaline magmas (panel a) and the high-La basalts (panel b). Model parameters are listed in Table 3. The modelling is done stepwise, but in reality the slab components may be generated and mix with the mantle wedge in any order. Both models first calculate a composite slab component from subducted sediment and igneous crust (mixing curve a). The curve is constrained to pass through the HNABs of central Mexico that define an end member in $\mathrm{Sr}-\mathrm{Nd}$ isotope mixing space. In order to pass through the observed data, model curve a assumes some (c. $15 \%$ ) loss of sedimentary $\mathrm{Sr}$ and preferential release (by a factor of 2) of $\mathrm{Sr}$ from igneous crust, which is in agreement with progressive slab dehydration (e.g. Kessel et al. 2005; Savov et al. 2005). In a second step, a composite of this slab fluid, which undergoes additional fractionation of $\mathrm{Sr} / \mathrm{Nd}$ by preferential retention of $\mathrm{Nd}$ in slab, creates a slab component with high $\mathrm{Sr} / \mathrm{Nd}$

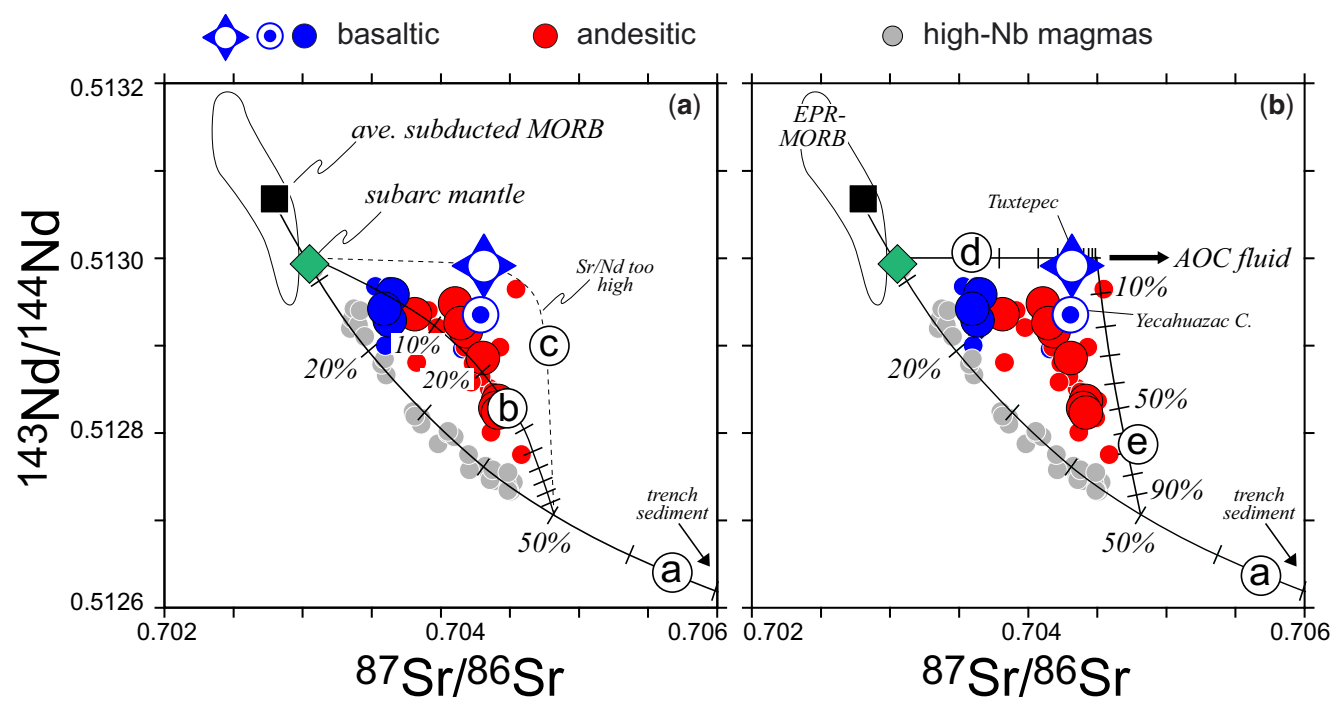

Fig. 14. ${ }^{87} \mathrm{Sr} /{ }^{86} \mathrm{Sr}$ v. ${ }^{143} \mathrm{Nd} /{ }^{144} \mathrm{Nd}$ mixing models for central MVB calc-alkaline basalts and andesites. Model parameters are compiled in Table 3. High-Nb arc magmas (grey circles) of the central MVB after Straub et al. (2013), LaGatta (2003) and this study. (a) Three-component mixing model for low-La calc-alkaline magmas, assuming Sr-rich fluid-like composite component from subducted sediment and igneous crust to infiltrate subarc mantle. (b) Four-component mixing model of high-La calc-alkaline basalts, assuming in addition an Sr-rich fluid with high ${ }^{87} \mathrm{Sr} /{ }^{86} \mathrm{Sr}$ from altered oceanic crust. Zero-age MORB from East Pacific Rise after PetDB (2011) Subducting igneous oceanic crust and terrigenous sediment from LaGatta (2003) and Gomez-Tuena et al. (2007a). 
Table 3. Summary of Sr and Nd isotope modelling

\begin{tabular}{|c|c|c|c|c|c|}
\hline & ${ }^{87} \mathrm{Sr} /{ }^{86} \mathrm{Sr}$ & ${ }^{143} \mathrm{Nd} /{ }^{144} \mathrm{Nd}$ & $\mathrm{Nd}$ & $\mathrm{Sr}$ & $\mathrm{Sr} / \mathrm{Nd}$ \\
\hline \multicolumn{6}{|l|}{ End member compositions } \\
\hline Terrigenous trench sediment $^{1}$ & 0.70846 & 0.51252 & 23.4 & 164.1 & 7.0 \\
\hline Subducting igneous crust ${ }^{2}$ & 0.70278 & 0.51307 & 12.28 & 123.8 & 10.1 \\
\hline Mantle wedge $\mathrm{s}^{3}$ & 0.70305 & 0.51300 & 1.25 & 19.9 & 15.9 \\
\hline \multicolumn{6}{|l|}{$\begin{array}{l}\text { Curve a } \\
\text { Mixing of subducted sedimen }\end{array}$} \\
\hline terrigenous trench sediment ${ }^{4}$ & 0.70846 & 0.51252 & 23.40 & $140^{4}$ & 6.0 \\
\hline subducting igneous crust ${ }^{5}$ & 0.70278 & 0.51307 & 12.28 & $250^{5}$ & 20.4 \\
\hline \multicolumn{6}{|c|}{ Curve $\boldsymbol{b}$} \\
\hline mantle wedge ${ }^{3}$ & 0.70305 & 0.51307 & 1.25 & 19.9 & 10.1 \\
\hline Composite slab component & 0.70482 & 0.51271 & $4^{6}$ & 195 & 48.8 \\
\hline \multicolumn{5}{|c|}{ Mixing of mantle and composite slab component } & \\
\hline mantle wedge ${ }^{3}$ & 0.70305 & 0.51307 & 1.25 & 19.9 & 10.1 \\
\hline Composite slab component & 0.70482 & 0.51271 & $0.2^{7}$ & 195 & 975 \\
\hline \multicolumn{6}{|c|}{ Curved } \\
\hline Mantle wedge & 0.70305 & 0.51300 & 1.25 & 19.9 & 10.1 \\
\hline Sr-rich fluid from igneous crust ${ }^{8}$ & 0.70450 & $0.51300^{8}$ & 12.28 & $200^{8}$ & 16.3 \\
\hline \multicolumn{6}{|c|}{ Curve $\boldsymbol{e}$} \\
\hline Pre-impregnated mantle wedge & 0.70450 & $0.51300^{8}$ & 12.28 & $200^{8}$ & 16.3 \\
\hline Composite slab component $^{9}$ & 0.70482 & 0.51271 & 17.84 & 195 & 10.9 \\
\hline
\end{tabular}

${ }^{1}$ Terrigenous trench sediment from DSDP Site 467 after LaGatta (2003).

${ }^{2}$ Isotope ratios estimated from average zero-age East Pacific Rise MORB (PetDB 2011); Sr and Nd abundances are for average EPR (Donnelly et al. 2004).

${ }^{3}$ Isotope ratios based on 'old Texcal Flow' from Straub et al. (2013), Sr and Nd abundances for primitive mantle from McDonough \& Sun(1995).

${ }^{4} \mathrm{Sr}$ reduced by $15 \%$, assuming early loss of sedimentary $\mathrm{Sr}$ beneath forearc.

${ }^{5} \mathrm{Sr}$ increased by a factor of c. 2, assuming preferential mobilization of Sr from igneous crust.

${ }^{6} \mathrm{Nd}$ reduced by $78 \%$, assuming retention of $\mathrm{Nd}$ in slab, thus creating a slab component with high $\mathrm{Sr} / \mathrm{Nd}$.

${ }^{7} \mathrm{Nd}$ reduced by $98 \%$, assuming near complete $\mathrm{Nd}$ retention in slab, thus creating a slab component with very high $\mathrm{Sr} / \mathrm{Nd}$.

${ }^{8}$ Assuming ${ }^{87} \mathrm{Sr} /{ }^{86} \mathrm{Sr}=0.7045$ and $\mathrm{Sr}=200 \mathrm{ppm}$ for fluid from altered oceanic crust.

${ }^{9}$ Assuming no additional fractionation of $\mathrm{Sr} / \mathrm{Nd}$ in composite slab component.

(c. 49) that mixes with the low $\mathrm{Sr} / \mathrm{Nd}$ mantle (c. 16) to produce arc magmas with $\mathrm{Sr} / \mathrm{Nd} c$. 20-30 (curve b). A still higher $\mathrm{Sr} / \mathrm{Nd}$ of the slab component would reproduce the extreme ${ }^{87} \mathrm{Sr} /{ }^{86} \mathrm{Sr}$ and ${ }^{143} \mathrm{Nd} /{ }^{144} \mathrm{Nd}$ of the high-La basalts (stippled curve c), but only at unrealistically high (not observed) $\mathrm{Sr} / \mathrm{Nd}, c .100$, of the arc magmas. On the other hand (Fig. 14b), the low Sr/Nd, c. 15-20, of the high-La Tuxtepec and Yecahuazac Cone basalts can be generated, assuming the existence of a Sr-rich slab fluid with $\mathrm{Sr} / \mathrm{Nd} c .16$ and high ${ }^{87} \mathrm{Sr} /{ }^{86} \mathrm{Sr}, c$. 0.7045 , from altered igneous crust (curve $\mathbf{d}$ in panel b). Mixing of a composite slab fluid with low, unfractionated $\mathrm{Sr} / \mathrm{Nd}, c$. 11 , may then create source with high ${ }^{87} \mathrm{Sr} /{ }^{86} \mathrm{Sr}$ at ${ }^{143} \mathrm{Nd} /{ }^{144} \mathrm{Nd}$, and the low $\mathrm{Sr} / \mathrm{Nd}$ of the high-La basalts (curve e).

In summary, mixing models show that - despite the deceptively limited range in ${ }^{87} \mathrm{Sr} /{ }^{86} \mathrm{Sr}$ and
${ }^{143} \mathrm{Nd} /{ }^{144} \mathrm{Nd}$ - the calc-alkaline magmas are a complex mixture of source components with similar ${ }^{87} \mathrm{Sr} /{ }^{86} \mathrm{Sr}$ and ${ }^{143} \mathrm{Nd} /{ }^{144} \mathrm{Nd}$ and different $\mathrm{Sr} / \mathrm{Nd}$. No single solution exists, and it is possible that each magma batch, regardless of whether it is erupted from the same volcano or from a different volcano, represents a slightly different mixture of source components.

\section{Implications for arc fluxes and arc crustal growth}

Overall, the results of our study show that there is nothing in the incompatible trace elements and ${ }^{87} \mathrm{Sr} /{ }^{86} \mathrm{Sr}$ and ${ }^{143} \mathrm{Nd} /{ }^{144} \mathrm{Nd}$ of the calc-alkaline magmas that would invalidate the pyroxenite model. Moreover, the results are in line with models that 
propose a strong causal link between andesite melt formation and plate subduction (Ringwood 1974; Defant et al. 1991; Kelemen 1995; Yogodzinski et al. 1995). There seems to be no need for a causal role of crustal differentiation. Clearly, as such the pyroxenite model of andesite genesis has far-reaching implications for connectivity between trench and arc, the magnitude of the arc outflux and arc crustal growth. These will be shortly discussed in the following.

The link between andesite genesis and plate subduction. The strength of the link between andesite formation and plate subduction is best seen in the major element oxides that form $>99 \%$ of the mass of the erupted arc magmas. If arc magmas were principally partial melts of 'reaction pyroxenites', then the increased melt $\mathrm{SiO}_{2}$ of the arc magmas reflects their origin of as hybrid of mantle and silicic slab components. Such connections should also affect the variations of other major elements. Major element oxides $\mathrm{TiO}_{2}, \mathrm{FeO}, \mathrm{MnO}, \mathrm{MgO}$ and $\mathrm{CaO}$ are not mobilized in the silicic slab component and must originate from the subarc mantle (e.g. Beard \& Lofgren 1991; Rapp \& Watson 1995; Kessel et al. 2005; Klimm et al. 2008; Skora \& Blundy 2010). Thus, they should - as observed correlate inversely with melt $\mathrm{SiO}_{2}$ (Fig. 2). In contrast, major element oxides that co-correlate with $\mathrm{SiO}_{2}$ in abundance space, such as $\mathrm{Na}_{2} \mathrm{O}$ and $\mathrm{K}_{2} \mathrm{O}$, should be slab derived. Moreover, $\mathrm{P}_{2} \mathrm{O}_{5}$ behaves similar to $\mathrm{Nd}$, and may also be partially recycled. As incompatible elements, $\mathrm{K}_{2} \mathrm{O}, \mathrm{Na}_{2} \mathrm{O}$ and $\mathrm{P}_{2} \mathrm{O}_{5}$ can be assessed by the inverse approach (Figs 10 \& 15). While the slab control for $\mathrm{K}_{2} \mathrm{O}$ is obvious, inversion reveals significant slab contributions to $\mathrm{Na}_{2} \mathrm{O}$ and $\mathrm{P}_{2} \mathrm{O}_{5}$ (Fig. 15). The case of $\mathrm{Al}_{2} \mathrm{O}_{3}$, however, is more difficult, because it cannot be

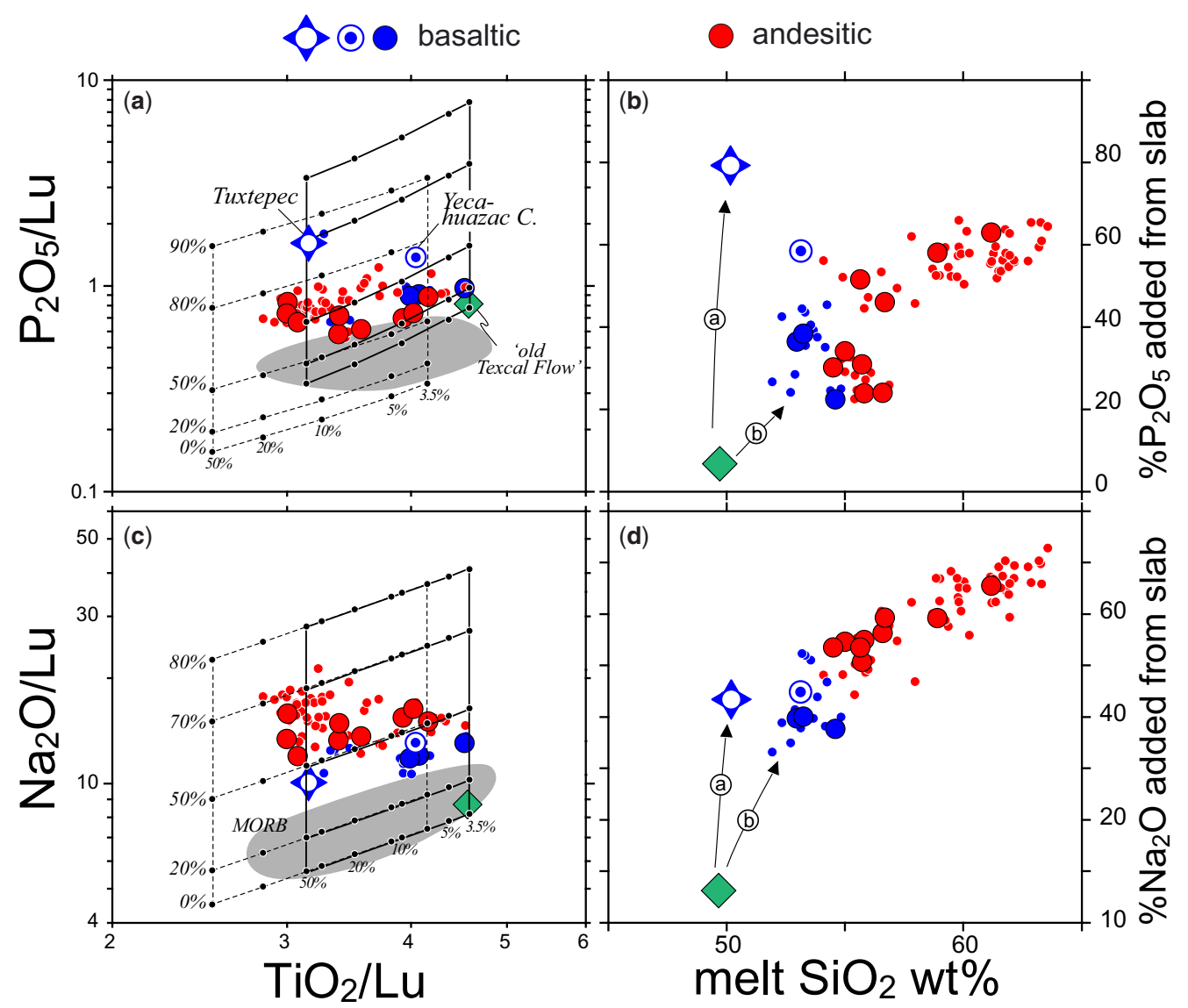

Fig. 15. (a, b) $\mathrm{Na}_{2} \mathrm{O} / \mathrm{Lu}$ and $\mathrm{P}_{2} \mathrm{O}_{5} / \mathrm{Lu}$ v. $\mathrm{TiO}_{2} / \mathrm{Lu}$, and (c, d) percentage of slab-derived elements $\mathrm{Na}_{\text {and }} \mathrm{P}_{2} \mathrm{O}_{5}$ in the arc magmas v. in central MVB calc-alkaline basalts and andesites. All samples have $\mathrm{Mg \#}>60$. The bulk partition coefficient of $\mathrm{Na}\left(D_{\mathrm{Na}}\right)$ is assumed to be that of $D_{\mathrm{TiO} 2}$ during mantle melting. All symbols and data sources as in Figures 10 and 12. 
assessed by the inverse approach. There is nothing in abundance space that would suggest $\mathrm{Al}_{2} \mathrm{O}_{3}$ recycling, but this is inconclusive. $\mathrm{Al}_{2} \mathrm{O}_{3}$ is a major component in alumosilicate slab melts (Beard \& Lofgren 1991; Rapp \& Watson 1995; Kessel et al. 2005), but also a significant constituent of the upper mantle. Thus, mantle melting may buffer the slab flux and obscure easy evidence of slab contributions of $\mathrm{Al}_{2} \mathrm{O}_{3}$ in arc magmas. In summary, despite some uncertainties, our extended dataset fully agrees with our earlier results that the major element oxides of the central MVB andesites were blends of slab and mantle materials, and did not result from shallow crustal processing of partial peridotite melts (Straub et al. 2008, 2013).

The effects of crustal differentiation. While the pyroxenite model does not require the overlying crust in order to create andesitic magmas, it does not imply the lack of subsequent intra-crustal differentiation. Without crustal differentiation, all central MVB magmas had high $\mathrm{Mg \#}>70$ numbers (Straub et al. 2011). However, the fact that most erupted magmas have lower values of $\mathrm{Mg \#}$ requires additional differentiation that should take place in the arc crust, which poses a physical and thermal barrier to the ascending mantle melts.

Because initial andesitic melts already have low melt $\mathrm{MgO}$ and $\mathrm{FeO}$, moderate loss of mafic silicates (olivine, pyroxenes) suffices to produce the low-Mg\# melts observed. Loss of mafic silicates will mostly affect melt $\mathrm{MgO}$, which is rapidly lowered. In contrast, the abundance levels of melt $\mathrm{SiO}_{2}$ and $\mathrm{FeO}$ - with bulk $D$ close to unity - are largely maintained (Straub et al. 2011). Olivine, the ubiquitous and often only phenocrysts of the high-Mg\# magmas, clearly plays a major role in lowering melt $\mathrm{Mg \#}$. However, results from melting experiments provide an interesting alternative as they demonstrate that, at the higher pressures in the lower crust, pyroxenes, not olivines, are on the liquidus of the andesitic melts (Weaver et al. 2011; Weber et al. 2011). While the presence of high-Mg\# pyroxenes in our sample set remains to be confirmed, there are revealing trends of olivine in Fo v. Ni space, where olivines from various lava flows are aligned in conspicuous sub-parallel trends. This is true for olivines from different volcanoes, but also for olivines of different eruptive series of single volcanic centres (exemplified for Guespalapa volcanics in Fig. 16a). Excepting rare cases where complex crustal intracrustal differentiation may play a role (Straub et al. 2011), there are two ways to create such trends: (1) the initial melts have different values of $\mathrm{Mg \#}$, either by inherent source heterogeneity or by progressive depletion through serial melting; or (2) the values of $\mathrm{Mg \#}$ of initial melts are variably lowered by early pyroxene fractionation in the lower crust, or even in the uppermost lithospheric mantle (Fig. $16 \mathrm{~b})$. This is because crystallizing pyroxenes also lowers melt $\mathrm{Mg \#}$ (if at a lesser rate than olivine),

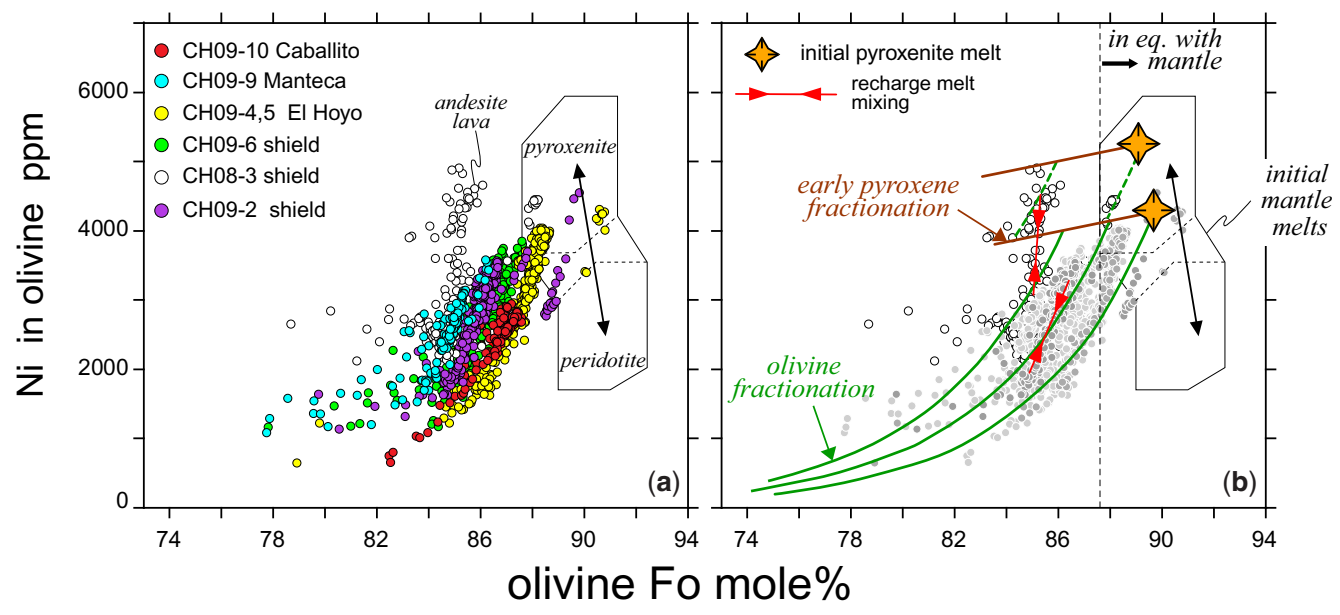

Fig. 16. (a) Olivine Ni (ppm) v. Fo (mol\%) for samples from Guespalapa Volcanic Complex. All olivine data are from Straub et al. (2011). Fields labelled 'pyroxenite' and 'peridotite' indicate olivine composition in equilibrium with initial partial melts of pyroxenite and peridotite after Straub et al. (2011). Large black arrow indicates mixing between pyroxenite and peridotite initial component melts. (b) Possible interpretation of olivine trends after Straub et al. (2011, 2013). Few olivine are in equilibrium with initial mantle melts (white field with hybrids of pyroxenite and peridotite melts), and most olivine crystallize from derivate melts. Thick brown lines delineate early pyroxene fractionation; thick green lines delineate olivine fractionation trends. Red lines with arrows indicate recharge mixing. 
but barely affect melt $\mathrm{Ni}$ given the much lower- $K_{\mathrm{d}}$ $\mathrm{Ni}$ in pyroxenes (e.g. Beattie et al. 1991). In particular, the higher the $\mathrm{SiO}_{2}$ of the initial melt, the more likely it is that pyroxenes will be on the liquidus. Such early pyroxenes may be left behind as residues at the walls of conduits walls through which the magmas ascend to surface.

Evidence for crustal melt processing is ubiquitous in the complex zoning patterns of olivines that are recorded in all calc-alkaline magmas mixing between two or more component melts (Straub et al. 2011). Given their low to moderate $\mathrm{H}_{2} \mathrm{O}$ abundances of the central MVB magmas (Cervantes \& Wallace 2003a, b; Johnson et al. 2009; Roberge et al. 2009), olivine will only crystallize in the upper crust. Thus, most melt mixing must happen in the upper crust, and probably occurs in the shallow magma plumbing system just prior to and during eruption. Much of this mixing is probably 'recharge mixing', where newly ascending melt batches charge into slower ascending or stagnant melts at higher level and ultimately trigger the eruptions (Kent et al. 2010). Clearly, early loss of mafic silicates and late-stage melt mixing will obscure the spectrum of initial melt that make up the central MVB calc-alkaline andesites (Straub et al. 2011). However, neither process seems efficient in entirely eliminating the characteristics of the initial mantle melts.

Magnitude of the arc outflux and connectivity to slab. The pyroxenite model also has consequences for the magnitude of the arc outflux. Models that assume a basaltic flux to the Moho calculate the arc outflux for the lower elemental concentration appropriate to basaltic melts (e.g. Plank \& Langmuir 1993; Straub \& Layne 2003). This limitation, however, does not exist for initial arc magmas produced by reaction pyroxenites that are more silicic and richer in many incompatible elements. Because the element concentrations of the initial arc magmas relate linearly to the magnitude of the arc outflux, the arc outflux of incompatible elements increases accordingly. This aspect is particularly intriguing for slab-controlled, recycled elements in arc magmas that tend to be concentrated in the silicic melts released from slab (e.g. highly incompatible mobile element like $\mathrm{Cs}, \mathrm{Ba}, \mathrm{Rb}, \mathrm{K}, \mathrm{U}, \mathrm{Th}$, $\mathrm{Pb}, \mathrm{Sr}$ ), but also for the recycled, climatically active volatile elements (e.g. $\mathrm{H}_{2} \mathrm{O}, \mathrm{CO}_{2}$, halogens). Moreover, being transported to surface in the $\mathrm{SiO}_{2^{-}}$ rich mantle melts obviates the need for extensive and laborious crustal differentiation, and ensures better connectivity between slab input and arc output. Revised estimates, based on the pyroxenite model, may significantly factor into models of the feedback between subduction cycling and the mechanisms of the ocean-atmosphere system.
Implications for arc crustal growth. The pyroxenite model has implications for models of arc crustal growth, as it offers the possibility that the arc crust may grow through accretion of a broad spectrum of basaltic, andesitic and dacitic mantle melts. Depending on the ratio of basaltic to dacitic melts (an unknown variable), the newly accreted crust may be andesitic on average. This would much reduce, and possibly even forgo, the periodic delamination of mafic crustal residues that is needed in order to create andesitic crust from initial basaltic melts (e.g. Tatsumi 2000). Furthermore, the pyroxenite model also affects the rates of arc crustal growth. One method to calculate arc crustal growth is to estimate the composition and volume of the extrusive magma series, and infer the volume of the mafic intrusives that must be left behind if the flux to the Moho was basaltic (Crisp 1984; White et al. 2006). However, this approach fails if the subarc mantle produces a broad range of mafic and silicic compositions, as in this case the eruptive volumes do not permit inferences on the volumes of the intrusive rocks. Rather, the intrusive volume is an unknown quantity, as there is no knowledge on how much of the respective basaltic, andesitic and dacitic mantle melts will erupt, and whether the erupted melt volumes will at least reflect the correct proportions of the initial melts. Kent et al. (2010) recently proposed that mafic melts (by their higher density) and dacitic-rhyolitic melts (by their viscosity) may preferentially become sequestered in the crust, while intermediate andesites preferentially erupt. There is also the possibility that the proportions of mafic $\mathrm{v}$. silicic mantle melts change among different arcs depending on the subduction parameters, or during the life-time of arcs that typically comprise tens of millions of years (Price et al. 2005; Condie 2007). Such concepts render it impossible to use the erupted volcanic volumes as a proxy for the total magmatic volume produced per increment of time. Alternative approaches are thus required to deduce the volume and accretion rates of intrusive bodies directly. Geophysical constraints on geothermal gradients in continental magmatic arcs (Rothstein \& Manning 2003) and on how arc surface heat flux changes in tandem with subduction parameters (e.g. convergence rate, Zellmer 2008, 2009) may help to provide some valuable insights in the future.

\section{Concluding remarks}

The major results of this study can be summarized as follows:

(1) The systematics of incompatible trace elements and ${ }^{87} \mathrm{Sr} /{ }^{86} \mathrm{Sr}$ and ${ }^{143} \mathrm{Nd} /{ }^{144} \mathrm{Nd}$ ratios of the central MVB calc-alkaline basalts 
to andesites agree with their proposed origin as partial melts from 'reaction pyroxenite' in the mantle wedge.

(2) Andesite formation in the central MVB is primarily a consequence of reactions between silicic slab components and the peridotite mantle, and not of intra-crustal melt differentiation.

(3) The pyroxenite model implies strong causal links between andesite genesis and plate subduction with implications for models of arc crustal growth, the efficiency of slab recycling and the connectivity between the arc magmatic fluxes and the Earth's exosphere.

Special thanks to V. L. Becerra, J. V. Gonzalez, H. S. H. Fernandez and S. N. Licona for a memorable sampling trip to Popo. R. Conrey, W.-Y. Hsu, D. Johnson and O. Pérez-Arvizu are thanked for laboratory support. G. Mesko helped with sample preparation, and J. Jweda, L. Mori, P. Sasnett and J. van Tongeren assisted in the field. Constructive reviews of two anonymous reviewers and the editorial support of Phil Leat are gratefully acknowledged. This study was financially supported by the US National Science Foundation (grant EAR-0738707 to S.M.S.) and the National Science Council of Taiwan (grants 96-2811-M-001-023 and 98-2811M-001-052 to G.F.Z.).

\section{References}

Agustín-Flores, J., Siebe, C. \& Guilbaud, M. N. 2011. Geology and geochemistry of Pelagatos, Cerro del Agua, and Dos Cerros monogenetic volcanoes in the Sierra Chichinautzin Volcanic Field, south of México City. Journal of Volcanology and Geothermal Research, 201, 143-162, http://dx.doi.org/10.1016/ j.jvolgeores.2010.08.010

Baker, M. B., Grove, T. L. \& Price, R. 1994. Primitive basalts and andesites from the Mt. Shasta region, N. California: products of varying melt fraction and water content. Contributions to Mineralogy and Petrology, 118, 111-129.

Ballentine, C. J. \& Burnard, P. G. 2002. Production, release and transport of noble gases in the continental crust. Reviews in Mineralogy and Geochemistry, 47, 481-538.

BEARD, J. S. 1995. Experimental, geological and geochemical constraints on the origins of low-Ks silicic magmas in oceanic arcs. Journal of Geophysical Research, 100, $15593-15600$.

Beard, J. S. \& Lofgren, G. E. 1991. Dehydration melting and water-saturated melting of basaltic and andesitic greenstones and amphibolites at 1, 3 and $6.9 \mathrm{kbar}$. Journal of Petrology, 32, 365-401.

Beattie, P., Ford, C. \& Russell, D. 1991. Partition coefficients for olivine-melt and orthopyroxene-melt systems. Contributions to Mineralogy and Petrology, 109, 212-224.

Cervantes, P. \& Wallace, P. J. 2003a. The role of $\mathrm{H}_{2} \mathrm{O}$ in subduction zone magmatism: new insights from melt inclusions in high-Mg basalts from central Mexico. Geology, 31, 235-238.

Cervantes, P. \& Wallace, P. J. 2003b. Magma degassing and basaltic eruption styles: a case study of V2000 year BP Xitle volcano in central Mexico. Journal of Volcanology and Geothermal Research, 120, 249-270.

Condie, K. C. 2007. Accretionary orogens in space and time. In: Hatcher, R. D., Jr., Carlson, M. P., McBride, J. H. \& Martínez Catalán, J. R. (eds) 4-D Framework of Continental Crust. Geological Society of America, Memoirs, Boulder, 200, $145-158$.

CRISP, J. A. 1984. Rates of magma emplacement and volcanic output. Journal of Volcanology and Geothermal Research, 20, 177-211.

Davidson, J., Turner, S., Handley, H., Macpherson, C. \& Dosseto, A. 2007. Amphibole 'sponge' in arc crust? Geology, 35, 787-790.

Defant, M. \& Drummond, M. 1990. Derivation of some modern arc magmas by melting of young subducted lithosphere. Nature, 347, 662-665.

Defant, M. J., Richerson, P. M. et AL. 1991. Dacite genesis via both slab melting and differentiation: petrogenesis of La Yeguada volcanic complex, Panama. Journal of Petrology, 32, 1101-1142.

Donnelly, K., Goldstein, S. L., Langmuir, C. H. \& Spiegelman, M. 2004. Origin of Enriched Ocean Ridge Basalt and Implications for mantle dynamics. Earth and Planetary Science Letters, 226, 347-366.

Eichelberger, J. C. 1978. Andesitic volcanism and crustal evolution. Nature, 275, 21-27.

Elliott, T., Plank, T., Zindler, A., White, W. \& Bourdon, B. 1997. Element transport from subducted slab to juvenile crust at the Mariana arc. Journal of Geophysical Research, 102, 14991-15019.

EsPinasa-Perena, R. 2006. Lava tubes of the Suchiooc Volcano, Mexico. Bulletin of the Association for Mexican Cave Studies, 17, 80.

Ferrari, L., Orozco-Esquivel, M. T., Manea, V. C. \& ManeA, M. 2011. The dynamic history of the TransMexican Volcanic Belt and the Mexico subduction zone. Tectonophysics, 522-523, 122-149, http://dx. doi.org/10.1016/j.tecto.2011.09.018

Gill, J. 1981. Orogenic Andesites and Plate Tectonics. Springer, Berlin.

Gómez-Tuena, A., LaGatta, A., Langmuir, C. H., Goldstein, S. L., Ortega-Gutiérrez, F. \& Carrasco-Nunez, G. 2003. Temporal control of subduction magmatism in the eastern Trans-Mexican Volcanic Belt: mantle sources, slab contributions, and crustal contamination. Geochemistry Geophysics Geosystems, 8, 8913.

Gómez-Tuena, A., Langmuir, C. H., Goldstein, S. L., Straub, S. M. \& Ortega-Gutierrez, F. $2007 a$. Geochemical evidence for slab melting in the Trans-Mexican Volcanic Belt. Journal of Petrology, 48, 537-562, http://dx.doi.org/10.1093/petrology/ eg1071

Gómez-Tuena, A., Orozco-Esquivel, M. T. \& Ferrari, L. 2007b. Igneous petrogenesis of the Trans-Mexican Volcanic Belt. In: Alaniz-Álvarez, S. A. \& NietoSamaniego, Á. F. (eds) Geology of México: Celebrating the Centenary of the Geological Society of México. 
Geological Society of America Special Papers, 422, 129-181, http://dx.doi.org/10.1130/2007.2422(05)

Gómez-Tuena, A., Laura Mori, L., Goldstein, S. L. \& Perez-Arvizu, O. 2011. Magmatic diversity of western Mexico as a function of metamorphic transformations in the subducted oceanic plate. Geochimica et Cosmochimica Acta, 75, 213-241, http://dx.doi. org/10.1016/j.gca.2010.09.029

Hildreth, W. \& Moorbath, S. 1988. Crustal contributions to arc magmatism in the Andes of Central Chile. Contributions to Mineralogy and Petrology, 98, 455-489.

Johnson, E. R., Wallace, P. J., Delgado-Granados, H., Manea, V. C., Kent, A. J. R., Bindeman, I. N. \& Donegan, C. S. 2009. Subduction-related volatile recycling and magma generation beneath central Mexico: insights from melt inclusions, oxygen isotopes and geodynamic models. Journal of Petrology, 50, 1729-1764, http://dx.doi.org/10.1093/petrology/ egp051

KAY, R. W. 1978. Aleutian magnesian andesites: melts from Subducted Pacific Ocean crust. Journal of Volcanology and Geothermal Research, 4, 117-132.

Kelemen, P. B. 1995. Genesis of high Mg\# andesites and the continental crust. Contributions to Mineralogy and Petrology, 120, 1-19.

Kent, A. J. R., Darr, C., Koleszar, A. M., Salisbury, M. J. \& Cooper, K. M. 2010. Preferential eruption of andesitic magmas through recharge filtering. Nature Geoscience, 3, 631-636, http://dx.doi.org/10.1038/ ngeo 924

Kessel, R., Schmidt, M. W., Ulmer, P. \& Pettke, T. 2005. Trace element signature of subduction-zone fluids, melts and supercritical liquids at $120-180 \mathrm{~km}$ depth. Nature, 437, 724-727, http://dx.doi.org/10. 1038 /nature 03971

KIM, Y., Clayton, R. W. \& JACKSON, J. M. 2010. Geometry and seismic properties of the subducting Cocos plate in central Mexico. Journal of Geophysical Research, 115, http://dx.doi.org/10.1029/2009JB006942

Klimm, K., Blundy, J. D. \& Green, T. H. 2008. Trace element partitioning and accessory phase saturation during $\mathrm{H}_{2} \mathrm{O}$-saturated melting of basalt with implications for subduction zone chemical fluxes. Journal of Petrology, 49, 523-553, http://dx.doi.org/10. $1093 /$ petrology/egn001

Koenig, S. \& Schuth, S. 2011. Deep melting of old subducted oceanic crust recorded by superchondritic $\mathrm{Nb} /$ Ta in modern island arc lavas. Earth and Planetary Science Letters, 301, 265-274, http://dx.doi.org/10. 1016/j.eps1.2010.11.007

LaGatta, A. B. 2003. Arc magma genesis in the Eastern Mexican Volcanic Belt. PhD thesis, Columbia University, New York.

Langmuir, C. H., Klein, E. M. \& Plank, T. 1992. Petrological systematics of mid-ocean ridge basalts: constraints on melt generation beneath ocean ridges. In: Morgan, J. P., Blackman, D. K. \& Sinton, J. M. (eds) Mantle Flow and Melt Generation at Mid-Ocean Ridges. Geophysical Monograph. American Geophysical Union, Washington, DC, 183-280.

Martinez-Serrano, R. G., SchaAf, P., Solids-Pichardo, G., Hernandez-Bernal, M. S., HernandeZTrevino, T., Morales-Contreras, J. J. \& Macias,
J. L. 2004. $\mathrm{Sr}, \mathrm{Nd}$ and $\mathrm{Pb}$ isotope and geochemical data from the Quaternary Nevado the Toluca volcano, a source of recent adakite magmatism, and the Tenango Volcanic Field. Journal of Volcanology and Geothermal Research, 138, 77-110.

McDonough, W. F. \& Sun, S. S. 1995. The composition of the Earth. Chemical Geology, 120, 223-253.

Meriggi, L., Macías, J. L., Tommasini, S., CaPra, L. \& Conticelli, S. 2008. Heterogeneous magmas of the Quaternary Sierra Chichinautzin volcanic field (central Mexico): the role of an amphibole-bearing mantle and magmatic evolution processes. Revista Mexicana de Ciencias Geológicas, 25, 197-216.

Morris, J. D., Leeman, W. P. \& Tera, F. 1990. The subducted component in island arc lavas: constraints from Be isotopes and B-Be systematics. Nature, 344, 31-36.

NiU, Y. \& BatizA, R. 1997. Trace element evidence from seamounts for recycled oceanic crust in the Eastern Pacific mantle. Earth and Planetary Science Letters, 148, 471-483.

Ortega-Gutiérrez, F., Elías-Herrera, M., GómezTuena, A., Mori, L., Reyes-Salas, M., MacíasRomo, C. \& Solari, L. A. 2012. Petrology of highgrade crustal xenoliths in the Chalcatzingo Miocene subvolcanic field, southern Mexico: buried basement of the Guerrero-Morelos platform and tectonostratigraphic implications. International Geology Review, 54, 1597-1634, http://dx.doi.org/10.1080/00206814. 2011.649956

Pardo, M. \& Suarez, G. 1995. Shape of the subducted Rivera and Cocos plate in southern Mexico: seismic and tectonic implications. Journal of Geophysical Research, 100, 12357-12373.

Pearce, J. A. \& Peate, D. W. 1995. Tectonic implications of the compositions of volcanic arc magmas. Annual Review of Earth and Planetary Sciences, 23, 251-285.

Pearce, J. A., Baker, P. E., Harvey, P. K. \& Luff, I. W. 1995. Geochemical evidence for subduction fluxes, mantle melting and fractional crystallization beneath the South Sandwich Island Arc. Journal of Petrology, 36, 1073-1109.

Perez-Campos, X., Kim, Y. H. et AL. 2008. Horizontal subduction and truncation of the Cocos Plate beneath central Mexico. Geophysical Research Letters, 35, 18303, http://dx.doi.org/10.1029/2008GL035127

Pertermann, M. \& Hirschmann, M. M. 2003. Anhydrous partial melting experiments on MORB-like eclogite: phase relations, phase compositions and mineral-melt partitioning of major elements at 23 GPa. Journal of Petrology, 44, 2173-2201.

PETDB 2011. Information System for Geochemical Data of Igneous and Metamorphic Rocks from the Ocean Floor, http://www.earthchem.org/petdb

Plank, T. 2005. Constraints from Thorium/Lanthanum on sediment recycling at subduction zones and the evolution of the continents. Journal of Petrology, 46, 921-944, http://dx.doi.org/10.1093/petrology/ egi005

Plank, T. \& Langmuir, C. H. 1988. An evaluation of the global variations in the major element chemistry of arc basalts. Earth and Planetary Science Letters, 90, 349-370. 
Plank, T. \& Langmuir, C. H. 1993. Tracing trace elements from sediment input to volcanic output at subduction zones. Nature, 362, 739-743.

Price, R. C., Gamble, J. A., Smith, I. E. M., Stewart, R. B., EgGins, S. \& WRIGHT, I. C. 2005. An integrated model for the temporal evolution of andesites and rhyolites and crustal development in New Zealand's North Island. Journal of Volcanology and Geothermal Research, 140, 1-24, http://dx.doi.org/10.1016/ j.jvolgeores.2004.07.013

RAPP, R. P. \& WATSON, E. B. 1995. Dehydration melting of metabasalt at 8-32 kbar: implications for continental growth and crust-mantle recycling. Journal of Petrology, 36, 891-931.

Reubi, O. \& Blundy, J. 2008. Assimilation of plutonic roots, formation of high-K 'Exotic' Melt inclusions and genesis of andesitic magmas at Volcan De Colima, Mexico. Journal of Petrology, 49, 2221-2243, http:// dx.doi.org/10.1093/petrology/egn066

Reubi, O. \& Blundy, J. 2009. A dearth of intermediate melts at subduction zone volcanoes and the petrogenesis of arc andesites. Nature, 461, 1269-1273, http://dx.doi.org/10.1038/nature08510

Ringwood, A. E. 1974. The petrological evolution of island arc systems. Journal of the Geological Society, London, 130, 183-204.

Roberge, J., Delgado-Granados, H. \& Wallace, P. J. 2009. Mafic magma recharge supplies high $\mathrm{CO}_{2}$ and $\mathrm{SO}_{2}$ gas fluxes from Popocatépetl volcano, Mexico. Geology, 37, 107-110.

Rothstein, D. A. \& Manning, C. E. 2003. Geothermal gradients in continental magmatic arcs: constraints from the eastern Peninsular Ranges batholith, Baja California, México. In: Johnson, S. E., PAterson, S. R., Fletcher, J. M., Girty, G. H., Kimbrough, D. L. \& MARTín-BARAJAs, A. (eds) Tectonic Evolution of North-Western México and the Southwestern USA. Geological Society of America Special Papers, 374, 337-354.

RUDNICK, R. 1995. Making continental crust. Nature, 378, $571-578$.

Ruscitto, D. M., Wallace, P. J., Cooper, L. B. \& Plank, T. 2012. Global variations in $\mathrm{H}_{2} \mathrm{O} / \mathrm{Ce}$ : 2. Relationships to arc magma geochemistry and volatile fluxes. Geochemistry Geophysics Geosystems, 13, Q03025, http://dx.doi.org/10.1029/2011GC003887

Savov, I. P., Ryan, J. G., D’Antonio, M., Kelley, K. \& Mattie, P. 2005. Geochemistry of serpentinized peridotites from the Mariana Forearc Conical Seamount, ODP Leg 125: implications for the elemental reycycling in subduction zones. Geochemistry Geophysics Geosystems, 6, http://dx.doi.org/10.1029/ 2004GC000777

SchaAf, P. \& Carrasco-Núñez, G. 2010. Geochemical and isotopic profile of Pico de Orizaba (Citlaltépetl) volcano, Mexico: insights for magma generation processes. Journal of Volcanology and Geothermal Research, 197, 108-122, http://dx.doi.org/10.1016/ j.jvolgeores.2010.02.016

Schaaf, P., Stimac, J., Siebe, C. \& Macias, J. L. 2005. Geochemical evidence for mantle origin and crustal processes in volcanic rocks from Popocatepetl and surrounding monogenetic volcanoes, central Mexico. Journal of Petrology, 46, 1243-1282.
Siebe, C., Rodriguez-Lara, V., SchaAf, P. \& Abrams, M. 2004a. Geochemistry, $\mathrm{Sr}-\mathrm{Nd}$ isotope composition, and tectonic setting of Holocene Pelado, Guespalapa and Chichinautzin scoria cones, south of Mexico City. Journal of Volcanology and Geothermal Research, 130, 197-226.

Siebe, C., Rodriguez-Lara, V., Schaaf, P. \& Abrams, M. 2004b. Radiocarbon ages of Holocene Pelado, Guespalapa, and Chichinautzin scoria cones, south of Mexico City: implications for archeology and future hazards. Bulletin of Volcanology, 66, 203-225, http://dx.doi.org/10.1007/s00445-003-0304-z

Siebe, C., Arana-Salinas, L. \& Abrams, M. 2005. Geology and radiocarbon ages of Tlaloc, Tlacotenco, Cuauhtzin, Hijo del Cuauhtzin, Teuhtli, and Ocusacayo monogenetic volcanoes in the central part of the Sierra Chichinautzin, Mexico. Journal of Volcanology and Geothermal Research, 141, 225-243.

Skora, S. \& Blundy, J. 2010. High-pressure hydrous phase relations of radiolarian clay and implications for the involvement of subducted sediment in arc magmatism. Journal of Petrology, 51, 2211-2243, http:// dx.doi.org/10.1093/petrology/egq054

Staudigel, H., Davies, G. R., Hart, S. R., Marchant, K. M. \& Sмiтh, B. M. 1995. Large scale isotopic Sr, $\mathrm{Nd}$ and $\mathrm{O}$ isotopic anomaly of altered oceanic crust: DSDP/ODP sites 417/418. Earth and Planetary Science Letters, 130, 169-185.

Stolper, E. \& Newman, S. 1994. The role of water in the petrogenesis of Mariana trough magmas. Earth and Planetary Science Letters, 121, 293-325.

Stolz, A. J., Jochum, K. P., Spettel, B. \& Hofmann, A. W. 1996. Fluid- and melt-related enrichment in the subarc mantle: evidence from $\mathrm{Nb} / \mathrm{Ta}$ variations in island-arc basalts. Geology, 24, 587-590.

Straub, S. M. \& Layne, G. D. 2003. The systematics of chlorine, fluorine and water in Izu arc front volcanic rocks: implication for volatile recycling in subduction zones. Geochimica et Cosmochimica Acta, 67, 4179-4203.

Straub, S. M. \& Martin-Del Pozzo, A. L. 2001. The significance of phenocryst diversity in tephra from recent eruptions at Popocatepetl volcano (Central Mexico). Contributions to Mineralogy and Petrology, 140, 487-510.

Straub, S. M. \& Zellmer, G. F. 2012. Volcanic arcs as archives of plate tectonic change. Gondwana Research, 21, 495-516, http://dx.doi.org/10.1016/j. gr.2011.10.006

Straub, S. M., LaGatta, A. B., Martin-Del Pozzo, A. L. \& LANGMuir, C. H. 2008. Evidence from high $\mathrm{Ni}$ olivines for a hybridized peridotite/pyroxenite source for orogenic andesites from the central Mexican Volcanic Belt. Geochemistry Geophysics Geosystems, 9, Q03007, http://dx.doi.org/10.1029/ 2007GC001583

Straub, S. M., Gomez-Tuena, A., Stuart, F. M., Zellmer, G. F., EsPinasa-Perena, R., CaI, M. Y. \& IIZUKA, Y. 2011. Formation of hybrid arc andesites beneath thick continental crust. Earth and Planetary Science Letters, 303, 337-347, http://dx.doi.org/10. 1016/j.epsl.2011.01.013

Straub, S. M., Gomez-Tuena, A. et AL. 2013. The processes of melt differentiation in arc volcanic 
rocks: insights from OIB-type arc magmas in the central Mexican Volcanic Belt. Journal of Petrology, 54, 665-701, http://dx.doi.org/10.1093/petrology/egs081

Sun, S. S. \& McDonough, W. F. 1989. Chemical and isotopic systematics of oceanic basalts: implications for mantle composition and processes. In: SAUNDERs, A. D. \& Norry, M. J. (eds) Magmatism in the Ocean Basins. Geology Society, London, Special Publications, 42, 313-345.

TAMURA, Y. \& TATSUmi, Y. 2002. Remelting of an andesitic crust as a possible origin for rhyolitic magma in oceanic arcs: an example from the Izu-Bonin Arc. Journal of Petrology, 43, 1029-1047.

TAtsumi, Y. 2000. Continental crust formation by crustal delamination in subduction zones and complementary accumulation of the enriched mantle I component in the mantle. Geochemistry Geophysics Geosystems, 1, http://dx.doi.org/10.1029/2000GC000094

Tollstrup, D., Gill, J. B., Kent, A. J. R., Prinkey, D., Williams, R., Tamura, Y. \& Ishizuka, O. 2010. Across-arc geochemical trends in the Izu-Bonin arc: contributions from the subducting slab, revisited. Geochemistry Geophysics Geosystems, 11, http://dx.doi. org/10.1029/2009GC002847

Urrutia-Fucugauchi, J. \& Martin del Pozzo, A. L. 1993. Implicaciones de los datos paleomagneticos sobra la edad de la sierra Chichnautzin, Cuenca de Mexico. Geofisica Internationale, 33, 523-533.

Verma, S. P. 1999. Geochemistry of evolved magmas and their relationship to subduction-unrelated mafic volcanism at the volcanic front of the central Mexican Volcanic Belts. Journal of Volcanology and Geothermal Research 93, 151-171.

Wallace, P. J. \& Carmichael, I. S. E. 1999. Quaternary volcanism near the Valley of Mexico: implications for subduction zone magmatism and the effects of crustal thickness variations on primitive magma compositions. Contributions to Mineralogy and Petrology, 135, 291-314.

Weaver, S. L., Wallace, P. J. \& Johnston, D. A. 2011. A comparative study of continental v. intraoceanic arc mantle melting:Experimentally determined phase relations of hydrous primitive melts. Earth and
Planetary Science Letters, 308, 97-106, http://dx. doi.org/10.1016/j.epsl.2011.05.040

Weber, R. M., Wallace, P. J. \& Johnston, A. D. 2011. Experimental insights into the formation of high-Mg basaltic andesites in the trans-Mexican volcanic belt. Contributions to Mineralogy and Petrology, 163, 825-840, http://dx.doi.org/10.1007/ s00410-011-0701-9

White, S. M., Crisp, J. A. \& SPERA, F. J. 2006. Long-term volumetric eruption rates and magma budgets. Geochemistry Geophysics Geosystems, 7, 1-20.

Woodhead, J. D., Eggins, S. M. \& Johnson, R. W. 1998. Magma genesis in the New Britain Island arc: further insights into melting and mass transfer processes. Journal of Petrology, 39, 1641-1668.

Woodhead, J. D., Hergt, J. M., Davidson, J. P. \& EgGINS, S. M. 2001. Hafnium isotope evidence for 'conservative' mobility during subduction zone processes. Earth and Planetary Science Letters, 192, 331-346.

Yogodzinski, G. M., Kay, R. W., Volynets, O. N., Koloskov, A. V. \& KaY, S. M. 1995. Magnesian andesite in the western Aleutian Komandorsly region: implications for slab melting and processes in the mantle wedge. Geological Society of America Bulletin, 107, 505-519.

Yogodzinksi, G., LeEs, J. M., Churikova, T. G., DorenDORF, F., Woerner, G. \& Volynets, O. N. 2001. Geochemical evidence for the melting of the subducting oceanic lithosphere at the plate edges. Nature, $\mathbf{4 0 9}$, 500-504.

Zellmer, G. F. 2008. Some first-order observations on magma transfer from mantle wedge to upper crust at volcanic arcs. In: ANnen, C. \& Zellmer, G. F. (eds) Dynamics of Crustal Magma Transfer, Storage and Differentiation. Geological Society, London, Special Publications, 304, 15-31, http://dx.doi.org/10. $1144 /$ SP304.2

Zellmer, G. F. 2009. Petrogenesis of Sr-rich adakitic rocks at volcanic arcs: insights from global variations of eruptive style with plate convergence rates and surface heat flux. Journal of the Geological Society, London, 166, 725-734. 